Portland State University

PDXScholar

$5-1-1970$

\title{
The propagation of light in a turbulent atmosphere
}

Karl-Hans Gunther Engert

Portland State University

Follow this and additional works at: https://pdxscholar.library.pdx.edu/open_access_etds Let us know how access to this document benefits you.

\section{Recommended Citation}

Engert, Karl-Hans Gunther, "The propagation of light in a turbulent atmosphere" (1970). Dissertations and Theses. Paper 917.

https://doi.org/10.15760/etd.917

This Thesis is brought to you for free and open access. It has been accepted for inclusion in Dissertations and Theses by an authorized administrator of PDXScholar. Please contact us if we can make this document more accessible: pdxscholar@pdx.edu. 
AN ABSTRACT OF THE THESIS OF Kar1-Hans Gunther Engert for the Master of Science in Applied Science presented May 22, 1970.

Title: The Propagation of Light in a Turbulent Atmosphere.

APPROVED BY MEMBERS OF THE THESIS COMMITTEE:

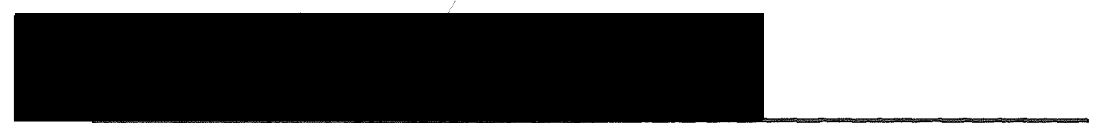

J. Richard Kerr, Chairman

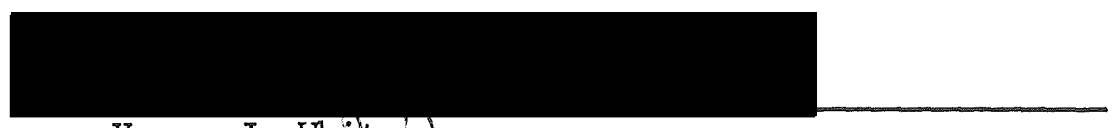

Harry J。 Whilte

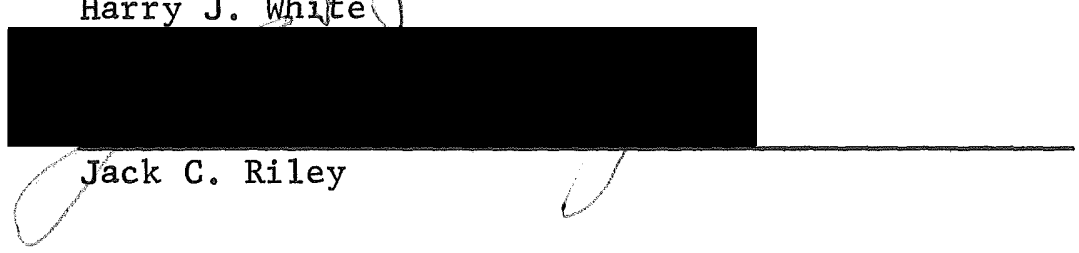

This report describes the instrumentation which is currently being utilized at the Oregon Graduate Center in a study of atmospheric turbulence effects on the long-range propagation of visible and infrared laser beams, with independent measurements of the turbulence parameters. The specially designed instrumentation includes portions of an Analog Computer; a special Differential Thermometer; a Laser Modulator; and auxiliary meteorological instruments; which were deve1oped by the author.

Sample experimental results are given in the report. 
THE PROPAGATION OF LIGHT IN A TURBULENT ATMOSPHERE

by

KARL-HANS GUNTHER ENGERT

A thesis submitted in partial fulfillment of the requirements for the degree of

MASTER OF SCIENCE

in

APPLIED SCIENCE

Portland State University

1970 
TO THE OFFICE OF GRADUATE STUDIES:

The members of the Committee approve the thesis of

Kar1-Hans Gunther Engert presented May 22, 1970.

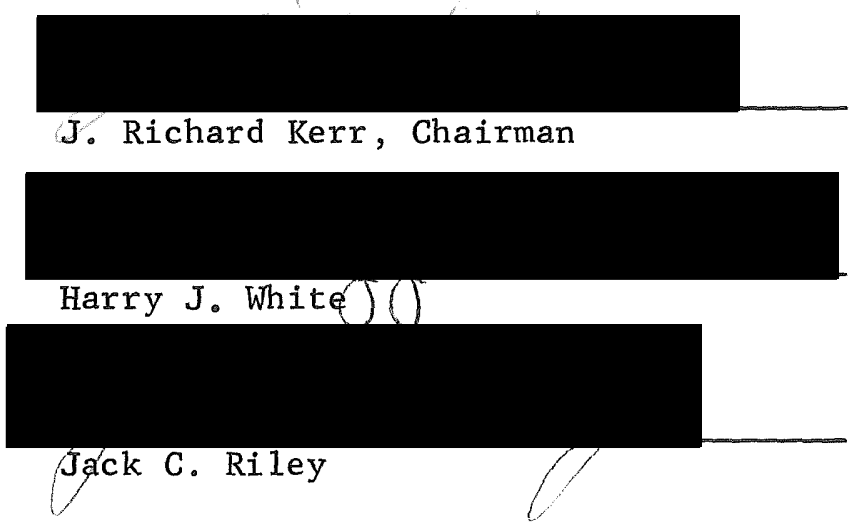

APPROVED:

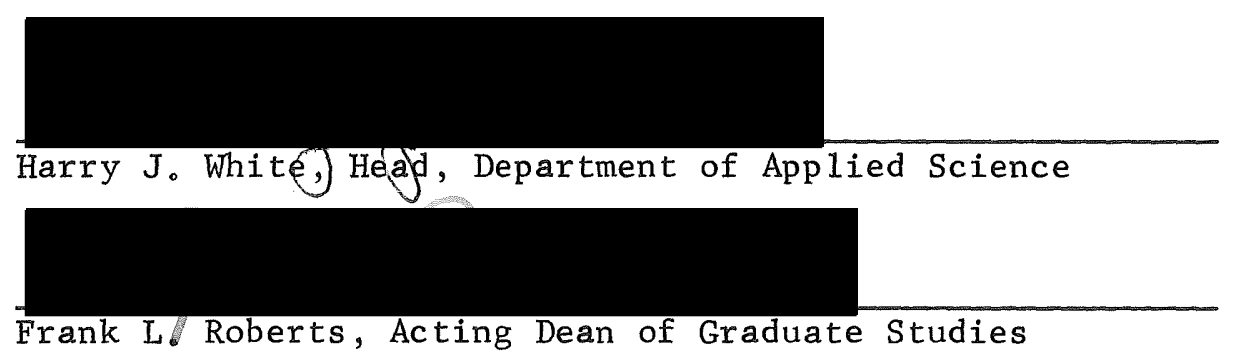

May 22,1970 


\section{ACKNOWLEDGMENTS}

This research was supported by the Advanced Research Projects Agency of the Department of Defense, and was monitored by the Office of Nava1 Research under Contract Number N00014-68-A-0461-0001.

I am indebted to Dr. David R. Malcolm, Dr. Harry J. White and Dr. Nan-Teh Hsu of Portland State University for the help and encouragement received in the continuation of my education.

My gratitude is due Dr. J. Richard Kerr for offering me an exciting Assistantship with the Oregon Graduate Center; for the suggestion of the interesting instrumentation project; and for his guidance during its execution.

Appreciation is expressed to Tektronix, Inc., for their cooperation during this time and for the use of their printing facilities. Finally, I wish to thank my wife for her encouragement and invaluable help in the preparation of the text and for typing the Thesis . 
TABLE OF CONTENTS

PAGE

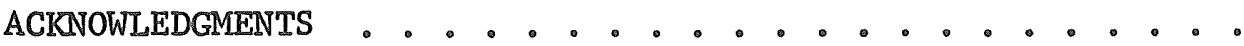

LIST OF TABLES . . . . . . . . . . . . . . . .

CHAPTER

INTRODUCTION . . . . . . . . . . 。

II OPTICAL PROPAGATION IN A TURBULENT ATMOSPHERE • .

A. Brief Survey of Developments in the Field of Atmospheric Turbulence . . .

B. Relationship Between Turbulence, Temperature Fluctuations and

Refractive Index ...........

C. Relationship Between Structure

Functions, Correlation Functions and

Spectral Density Functions . . . . . .

D. Propagation Theory Resu1ts .......

E. Research into Turbulence Propagation Phenomena at the Oregon Graduate Center .

III EXPERIMENTAL FACILITY FOR THE INVESTIGATION OF TURBULENCE PROPAGATION PHENOMENA ELECTRO-OPTICAL SYSTEMS ....................

A. Three-Wavelength Propagation Link ....

B. Genera1 Description of Signa1 Processing .

C. Detectors and Preamplifiers ......

D. Precision Demodulator ........

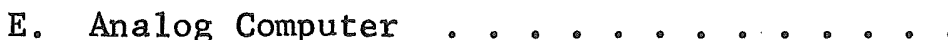


F. Precision Logarithmic Amplifiers . . . .

G. Portable Laser System ... . . . . . 44

H. Experimenta1 Results - Optica1 ..... 47

IV EXPERIMENTAL FACILITY FOR INVESTIGATION OF TURBULENCE PROPAGATION PHENOMENA

METEOROLOGICAL SYSTEMS - . . . : . . . . . • •

A. Differential Thermometer . . . . . .

Genera1 Description

Bridge Circuit

Preamplifier and Noise Performance Probe Drive Power and Influence of Air Velocity

Buffer and Variable Gain Amplifier

Demodulator and Filter

Squaring and Averaging

Feedback Stabilization

Maintenance and Operation

Calibration

B. Genera1 Meteorologica1 Instrumentation . .

Relative Humidity Indicator

Anemometer

Pressure and Temperature Indicators

C. Experimental Results ..........

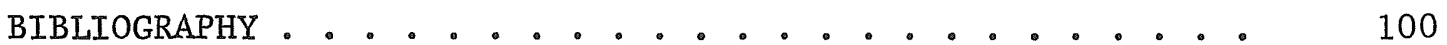

APPENDIX A Simple Logarithmic Converter with Gain using One Operational Amplifier . . . .

B Stability of the Logarithmic Converter. .

C Calculation of the Maximum Admissible Diode Saturation Current in the Absence of Noise .............

D Amplitude Calibrator for the Scintillation Computer . . . . . . . . . . . 
E Loop Gain Compensation and Stability in the $\mathrm{C}_{\mathrm{n}}$ Unit ..............

F Effect of the Wire Probe Diameter and Length on the Sensitivity of the $\mathrm{C}_{\mathrm{n}}$ Unit . . . . . . . . . . . .

G Repair of Damaged Thin Probe-Wires ....

H Large Time Constant in Feedback Integrators. $.0^{\circ} \cdot 0^{\circ} \cdot 0^{\circ} \cdot 0^{\circ}$ 


\section{LIST OF TABLES}

TABLE

PAGE

I Logarithmic Conversion, Typica1 Result . . . .

II Noise Measurements on Operational Amplifiers . .

III Microthermometer Squaring Module Performance . .

IV Sample Results: $\mathrm{C}_{\mathrm{n}}^{2}$ Determination by Three Methods .............. 
LIST OF FIGURES

FIGURE

PAGE

1. Structure Functions vs Separation . . . . . . .

2. Scintillation Variances . . . . . . . . . .

3. Oregon Graduate Center's Laser Range .......

4. Optica1 Receivers and Preamplifiers . . . . . .

5. Computer Block Diagram . . . . . . . . . .

6. Light Receiver and Preamplifier, IR . . . . . . .'

7. Light Receiver and Preamplifier, Visible Light..

8. Precision Demodulator . . . . . . . . . . .

9. Diode Current vs Voltage Characteristics,

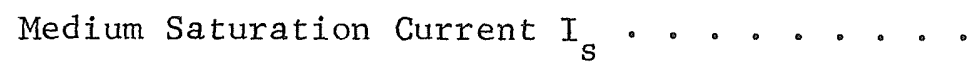

10. Diode Current vs Voltage Characteristics,

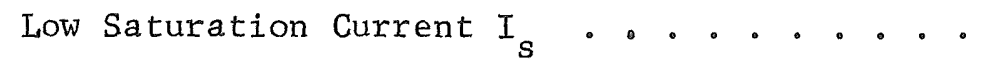

11. Precision Demodulator Symmetry Adjustment . . . .

12. Demodulator, Test Jack Output Voltage . . . .

13. Input and Output Voltages of Precision Demodulator . . . . . . . . . . . .

14. Logarithmic Converter and Voltage Amplifier . . .

15. Modulated Carrier, Demodulated and Logarithmic

Signals . . . . . . . . . . . . . .

16. Laser Modulator Circuit Diagram . . . . . . .

17. Linear Three Wavelength Scintillations . . . . . 
18. Logarithmic Three Wavelength Scintillations . . 50

19. Probability Distribution of Scintillation .... 51

20. Cumulative Probability vs Intensity,

Three Wavelengths ...........

21. Simultaneous Three Wavelength Scintillation

Spectra ................

22. Simultaneous Three Wavelength Covariance of

$$
\text { Scintillation ............ }
$$

23. Graph of Experimental and Theoretical Values of

$$
\text { Structure Constant } \mathrm{C}_{\mathrm{n}}{ }^{2} \text { vs Fresnel Number... }
$$

24. Refractive Index Structure Constant Meter .... 58

25. Block Diagram of $\mathrm{C}_{\mathrm{n}}{ }^{2}$ Instrument ........ 60

26. $\mathrm{C}_{\mathrm{n}}{ }^{2}$ Detector Bridge with Sensitivity Test

$$
\text { Circuit .............. }
$$

27. Frequency Response Test Wave Forms, Low End ...

28. Frequency Response Test Wave Forms, High End ...

29. Frequency Response of $\mathrm{C}_{\mathrm{n}}{ }^{2}$ Unit ........

30. Gain Cancellation in Control Loop of $\mathrm{C}_{\mathrm{n}}{ }^{2}$ Unit .

31. Photoresistance and Branch Resistance vs

$$
\text { Lamp Vo1tage and Bridge Sensitivity vs }
$$

Photoresistance ............

32. Graphs of Magnitude and Phase Angle vs

$$
\text { Frequency .............. }
$$

33. Composition of Open-Loop Gain, Bridge Balance

Control ............... 
34. Stability of Bridge Balancing Contro1 Loop .... 84

35. Relative Humidity Indicator and Recorder . . . 88

36. Glycerin Content of G1ycerin-Water Mixture vs Relative Humidity and Specific Gravity . . . 89

37. Relative Humidity vs Recorder Chart Divisions . . 89

38. Relative Humidity Indicator and Recorder of Improved Linearity . . . . . . . . 91

39. Stable, Low-Distortion, Sine-Wave Generator ... . 92

40. Anemometer Demodulator Circuit Diagram . . . . 94

41. Typica1 Turbulence-Modulated Carrier Signals . . 95

42. Cumulative Probability of Temperature Difference • 96

43. Recording of $\mathrm{C}_{\mathrm{n}}{ }^{2}$ and Scintillation Variances vs Time ............... . 99

44. Single-Operational-Amplifier Log-Converter . . . 104

45. Logarithmic Converter Open-Loop Gain . . . . . 106

46. Logarithmic Converter, Corrected Open-Loop Gain . . . . . . . . . . . 108

47. Amplitude Calibrator for Logarithmic Converter . . 113

48. Gain Cancellation in Control Loop of $\mathrm{C}_{\mathrm{n}}{ }^{2}$ Unit . . 115 


\section{CHAPTER I}

\section{INTRODUCTION}

The author has been involved in the state-of-the-art instrumentation of an experimental facility at the Oregon Graduate Center, at which laser propagation through the atmosphere is being investigated under a grant from the Office of Naval Research. This report describes mainly the author's contributions.

In Chapter II, the history of the development of turbulence theory is reviewed and pertinent terms and their interrelationships are explained. The results of turbulence theory relevant to this report are summarized. Finally, the research at the Oregon Graduate Center into propagation phenomena in the turbulent atmosphere is discussed.

In Chapter III, the optical facility is considered in detail. Then follows a discussion of a low-noise Precision Demodulator and a stable Logarithmic Converter, to which the author devoted much effort. Next is a description of a simple Electronic Square-Wave Laser Modulator. Sample results concerning portions of the instrumentation, and of the overall facility, are given.

Chapter IV is devoted to the discussion of a thermal method of evaluation for the refractive index structure function. A highlysensitive Differential Thermometer, in part designed by the author, is described in detail, and its sensitivity limitations are developed. A novel Relative Humidity Indicator, an Anemometer, and a Wind-Direction 
Indicator are described, and auxiliary meteorological recorders are mentioned. This Chapter concludes with the quotation of experimental results achieved with this instrumentation.

The report concludes with a Bibliography, rather than a list of References, because the former is believed to better serve the reader for information. Several Appendices elucidate in further detail some aspects and ideas. 
OPTICAL PROPAGATION IN A TURBULENT ATMOSPHERE

In the last fifteen years, we have experienced a constantly increasing effort towards the establishment of a solid theoretical foundation of turbulence pehnomena. The meteorologist is interested because he would like to predict weather better. The air pollution expert has to know the laws which govern the dispersal of smog and smoke (1). The flier, the radar man and the specialist in optical communications are all interested in different manifestations of turbulence.

As man succeeded in producing coherent light, he became interested in its vast information-carrying capabilities. Shannon's information Theorem predicts a bandwidth of $B=5 \times 10^{14}$ Hertz, which coherent red light can handle in principle (2). Unfortunately, as a laser beam progresses through turbulence and scatterers, its coherence deteriorates and the information channel becomes the determining factor in the prospect of multiplexed transmission of many simultaneous television programs and telephone channels.

The question as to what turbulence is has been studied by many men for various reasons; their work laid the foundation for present research efforts. In this Chapter, we review the contributions of a few of these men. Some of the relevant terms and relationships concerning turbulence and coherent propagation are discussed, in order to place 
in perspective the instruments which have been developed and the experiments being performed at the Oregon Graduate Center in optical propagation through a turbulent atmosphere.

\section{A. BRIEF SURVEY OF DEVELOPMENTS IN THE FIELD OF ATMOSPHERIC TURBULENCE}

In the following survey we briefly review the significant contributions and trace some of the developments pertinent to this Thesis.

Goedecke (3) reported measurements of atmospheric turbulence in 1936, at the beginning of a new wave of increasing activity in this field. One year later Rytov (4), often quoted for his controversial single-scattering approach, worked on the propagation of ultrasound, and proposed a method for solving the wave equation in the presence of large perturbations. In 1939, Loitsyanski (5) published his Basic Laws of Isotropic Turbulence.

An extensive series of publications on the theory of turbulent flow and related topics was begun in 1941 by Obukhov (6) and Kolmogorov (7), to whom the concept of a structure function, a statistical quantity measuring turbulence, is due (Chapter II-B). Batchelor's (8) Theory of Homogeneous Turbulence appeared in 1953. Up to that time, when Tatarski (9) postulated the normal distribution of the logarithmic amplitude of optical scintillation, a number of papers were concerned with sound propagation.

In 1960, Zwang (10) measured temperature pulse frequency spectra with a sensitive, automatically balanced microthermometer covering frequencies from 0.05 to $1160 \mathrm{Hertz}$. His results show the dependence of 
the spectra on meteorological conditions, and agreement with the "twothirds 1aw" (Chapter II-B) of the Obukhov-Kolmogorov theory.

Hufnagel (11) and Stanley surveyed the existing theory and developed a modulation transfer function for image transmission through a turbulent atmosphere in 1963. A dependence of the results on angular divergence of the light source was noted. Two years later, in 1965, Davis (12) experimented with three wavelengths, ranging from 0.7 to 10 micrometers, and applied the results to the calculation of the apertures and beam divergence to be used for a communications link with a specified modulation index and signal-to-noise ratio. He found Tatarski's formulae applicable for beam cross sections in excess of the product of the wavelength with the distance from the transmitter.

In 1967, Fried (13) further developed the complex structure function uniting the phase- and log-amplitude structure functions, and calculated the distortion of the equiphase surface, from which he derived the largest possible detector aperture for optical heterodyne reception. (It is interesting to note the analogy with electrical signals, which are often plotted on log-frequency abscissa and log-amplitude ordinate; and their phase is plotted on a linear ordinate with the same abscissa as above.) DeWolf (14) proposed the use of a Rayleigh or Rice-Nakagami distribution instead of the log-normal distribution of the scintillation amplitude measured on a sampled laser beam.

Ochs (15) used two highly sensitive cold-wire thermometers with the two probes arranged at variable vertical separations from each other to demonstrate on a cathode ray tube-XY display the correlation of the temperature fluctuations and its dependence on spacing. In a 
more recent letter, results of further experiments are reported (16), which include the propagation of a $6328 \AA$ laser beam over distances from 5 to 145 kilometers. The log-amplitude distribution was found to be normal, as predicted by theory. Earlier results concerning the refractive index structure functions are confirmed by these more extensive tests.

Wright (17) and Schutz measured thermal fluctuations with horizontally spaced cold-wire probes, in order to derive the refractive index structure function. They found it impossible to express it directly in the form of Tatarski's results $(9, \mathrm{p} .188)$ and pointed out the need for the collection of data on additional meteorological parameters to explain the discrepancies.

In a proposal to the Office of Naval Research, submitted in December 1967, Kerr (18) reviewed the large body of scientific publications dealing with atmospheric propagation and turbulence, and mapped out a research plan. The fallacies of an incorrect turbulence theory were pointed out and an extensive list of references on the subject was compiled.

After spending time in Russia with the Institute of Atmospheric Physics, Strohbehn (19) published a review of the recent developments in theory and experimentation in this field. The Rytov approach (Chapter II-B) was found to be valid in a wide range of lower frequencies, but not in the visible spectrum. Like Kerr (20), he also pointed out the necessity of a multiple wavelength experiment over a reasonable range of frequencies in order to verify some of the results of the theory. 
Livingston, Dietz and Alcaraz (21) recently measured thermal fluctuations versus probe spacing. The resulting spectra were in agreement with Zwang's findings, although the expected " $2 / 3$ power" dependence of the temperature variance or temperature structure function does not fit the results obtained at spacings differing by a factor of more than 3.6. Photographs of the cross section of a laser beam were taken at a long distance from the source, enabling an analysis for all directions in the transverse plane, and eliminating wind as a parameter. The intensity fluctuations were found to be isotropic when the beam was far enough above ground.

\section{B. RELATIONSHIP BETWEEN TURBULENCE, TEMPERATURE FLUCTUATIONS AND REFRACTIVE INDEX}

Turbulence is generated when wind blows over a mechanical obstacle or when warm air bubbles rise while the cooler surrounding air moves to fill the space left by them. The resulting eddies cause turbulence in turn, handing their energy down to eddy currents which they have generated. The energy is thus passed on to ever smaller eddies until, at last, frictional effects become important to the smallest, and the energy is transformed back into heat.

If we let $(\varepsilon)$ denote the rate at which heat is thus appearing, then since no dissipative effects occur during the cascading process, the energy passes through all other size eddies to the smallest at a rate which is $\varepsilon$. At any point this energy is given by $\left(\mathrm{mv}^{2} / 2\right)$ where $\mathrm{m}$ is the mass of air and $\mathrm{v}$ is the velocity. The energy transferred from larger to smaller perturbations per unit time is then proportional to $\left(v^{2} / t\right)$. Now, for $(r)$ a small distance, we have 


$$
1 / t=v / r
$$

and the energy transferred per unit time is proportional to $\left(v^{3} / r\right)$. Hence,

$$
v^{3} / r \geqslant \varepsilon
$$

An order-of-magnitude agreement is obtained if, instead of velocity, we use its fluctuation from the mean ( $\left.\mathrm{v}^{\prime}\right)$. A commonly used measure for the random fluctuations is the velocity variance, which is obtained by averaging the square of this fluctuation. This variance is related to $(E)$ and $(r)$ by the above argument $(9, p .29)$ :

$$
\left\langle\left(v^{\prime}\right)^{2}\right\rangle=(\varepsilon r)^{2 / 3}
$$

The velocity "structure function" is the variance of the difference of the velocities at two points taken as a function of their separation r. From (2-3) it may be argued that the velocity structure function $\mathrm{D}_{\mathrm{v}}$ is given by:

$$
D_{v}(r)=\left\langle(\Delta v)^{2}\right\rangle \propto r^{2 / 3}
$$

This constitutes the basis of the "2/3 1aw".

Al1 structure functions as introduced by Kolmogorov have in common this relationship with the separation $r$. The temperature structure function and the refractive index structure function are consequently defined as

$$
\begin{aligned}
& D_{T}(r)=\left\langle(\Delta T)^{2}\right\rangle=C_{T}{ }^{2} r^{2 / 3} \\
& D_{n}(r)=\left\langle(\Delta n)^{2}\right\rangle=C_{n}{ }^{2} r^{2 / 3}
\end{aligned}
$$

where the structure constants $C_{T}$ and $C_{n}$ depend on the vertical elevation and the meteorological and diurnal conditions. The ratio of the 
variances $D_{n} / D_{T}$ approaches a constant, when the temperature variations are sma11, and pressure can be taken to be constant. In this case $(\mathrm{dn} / \mathrm{dT})^{2}$, the squared derivative of the refractive index with respect to temperature, can be substituted for $D_{n} / D_{T}$ (15). The derivative $\mathrm{dn} / \mathrm{dT}$ may now be calculated from the formula for the refractive index of air (see Chapter II-C). For greater clarity, the dependence on relative humidity has been dropped:

$$
\mathrm{n}-1=\frac{79 \mathrm{p} 10^{-6}}{\mathrm{~T}}
$$

The derivative with respect to temperature is:

$$
\frac{\mathrm{dn}}{\mathrm{d} T}=\frac{-79 \mathrm{p} 10^{-6}}{\mathrm{~T}^{2}}
$$

Since only the magnitude of the structure functions and the structure constants is relevant, the sign of the differential is dropped. Division of $(2-6)$ by $(2-5)$ gives:

$$
\frac{D_{n}}{D_{T}}=\frac{C_{n}^{2}}{C_{T}^{2}}
$$

from which follows:

$$
\mathrm{C}_{\mathrm{n}}^{2}=\mathrm{C}_{\mathrm{T}}^{2}(\mathrm{dn} / \mathrm{dT})^{2}
$$

Finally, using $(2-5)\left(\mathrm{C}_{\mathrm{T}}{ }^{2}=\left\langle(\Delta \mathrm{T})^{2}\right\rangle \mathrm{r}^{-2 / 3}\right)$, the refractive index structure constant is obtained:

$$
C_{n}^{2}=\left\langle(\Delta T)^{2}\right\rangle \frac{\left(79 p 10^{-6}\right)^{2}}{T^{4}} r^{-2 / 3}
$$

The variance $\left\langle(\Delta \mathrm{T})^{2}\right\rangle$ can be measured by means of a differential 
thermometer. Such an instrument has been constructed by the author and will be described in Chapter IV.

\section{RELATIONSHIP BETWEEN STRUCTURE FUNCTIONS, CORRELATION FUNCTIONS AND SPECTRAL DENSITY FUNCTIONS}

The correlation function $\mathrm{B}_{\mathrm{F}}\left(\mathrm{r}_{1}, \mathrm{r}_{2}\right)$ is defined as follows:

$$
B_{F}\left(r_{1}, r_{2}\right) \equiv \overline{F_{r}\left(r_{1}\right) F_{r}\left(r_{2}\right)}
$$

where:

$$
F_{r}\left(r_{1}\right) \equiv f\left(r_{1}+r\right)-f\left(r_{1}\right)
$$

and likewise for $\mathrm{F}_{\mathrm{r}}\left(\mathrm{r}_{2}\right)$. By analogy with an expansion in terms of perfect squares, $(2-12)$ can be written:

$$
\begin{aligned}
& \overline{F_{r}\left(r_{1}\right) F_{f}\left(r_{2}\right)}=\frac{1}{2}\left[\overline{\left[f\left(r_{1}+r\right)-f\left(r_{2}\right)\right]^{2}}+\overline{\left[f\left(r_{1}\right)-f\left(r_{2}+r\right)\right]^{2}}\right. \\
& \overline{-\left[f\left(r_{1}+r\right)-f\left(r_{2}+r\right)\right]^{2}}-\overline{\left[f\left(r_{1}\right)-f\left(r_{2}\right)\right]^{2}}
\end{aligned}
$$

The four averaged square terms are called "Structure Functions" (9). In a stationary, isotropic field the correlation function depends only on the separation $r$ and not on the location of the two points, defined by $r_{1,2}$ and we can rewrite the left side of $(2-12)$ simply as $B_{F}(r)$.

The correlation function $\mathrm{B}_{\mathrm{F}}(r)$ can be expanded as a threedimensional Fourier integral $(9, \mathrm{p} .16)$ of the spectral density function $\emptyset(k)$, where $k$ represents spatial frequency. The pair of transforms for the isotropic case is listed below (9, p.17): 


$$
\begin{aligned}
& \emptyset(k)=\frac{1}{2 \pi^{2} k} \int_{0}^{\infty} r B_{f}(r) \sin (k r) d r \\
& B_{f}(r)=\frac{4 \pi}{r} \int_{0}^{\infty} k \emptyset(k) \sin (k r) d k
\end{aligned}
$$

Next, we consider the dielectric permittivity of the atmosphere. The information-transmitting beam finds its communication channel constituted by randomly sized and shaped blobs, in which density (molecules $/ \mathrm{m}^{3}=\mathrm{s}$ ) and temperature are related by the ideal gas law $(\mathrm{pV}=\mathrm{NkT})$, and pressure $(\mathrm{p})$ can be taken constant in view of its propagation with the speed of sound.

The relationship between temperature, dielectric constant and refractive index of the atmosphere can be demonstrated by expressing the density as the number of molecules (N) in a given volume (V), realizing that each contributes to the dielectric polarization ( $P$ ) when an electric field (E) is applied, thus giving rise to an electric excitation density (D) (Displacement vector):

$$
\vec{D}=\epsilon_{0} \vec{E}+\vec{P}
$$

where $\epsilon_{0}$ is the permittivity of free space $\left(8.86 \times 10^{-12}\right.$ Farad/meter). The density (s) can be written, using the ideal gas law, as follows:

$$
\mathrm{s}=\mathrm{N} / \mathrm{V}=\mathrm{p} / \mathrm{kT}
$$

If the polarization is related to the density by the molecular polarizability $(\propto)$ so that:

$$
\overrightarrow{\mathrm{P}}=\overrightarrow{\alpha s}
$$


then the polarizability $\left(X=P / E_{0} E\right)$ can be used to express the dielectric constant (K):

$$
\mathrm{K}=1+\alpha \mathrm{s} / \varepsilon_{\mathrm{O}} \mathrm{E}=1+\alpha \mathrm{p} / \mathrm{kT} \epsilon_{\mathrm{o}} \mathrm{E}
$$

In this form its dependence on temperature is evident.

The contribution of humidity present in the air to the dielectric constant can be accounted for by adding another term to equation (2-8). Debye (24) gives the polarizability of the water as:

$$
\alpha_{w}=\frac{a}{3}+\frac{\mu^{2}}{3 k T}
$$

where the constant $a$ is due to the distortion of the water molecule when an electric field is applied; $\mu$ is the dipole moment of the water molecule, $k$ is Boltzmann's constant and $T$ is the temperature. From thermodynamics we know that the total pressure of a gas mixture equals the sum of the partial pressures (p)

$$
P=\sum P_{i} \quad i=1,2 \ldots
$$

and the ratios of the partial pressure of two components ( $i$ and $j$ ) and of their corresponding numbers ( $n$ ) of molecules in a given volume are equa 1:

$$
P_{i} / P_{j}=n_{i} / n_{j} \quad \begin{aligned}
& i \\
& j=1,2 \ldots
\end{aligned}
$$

Therefore, equation (1-6) also holds for the partial density:

$$
s_{i}=n_{i} / V=P_{i} / k T \quad i=1,2 \ldots
$$

so that the dielectric constant for the atmosphere containing water vapor can be written, with the "partial" molecular polarizabilities $\alpha_{a}$ 
and $\alpha_{w}, p_{a}$ and $p_{w}$ the partial pressures of dry air and water vapor respectively:

$$
\begin{aligned}
& \mathrm{K}=1+\frac{1}{\mathrm{kT} \varepsilon_{\mathrm{O}} \mathrm{E}}\left(\alpha_{\mathrm{a}_{\mathrm{a}} \mathrm{P}}+\alpha_{w} \mathrm{P}_{\mathrm{w}}\right) \\
& \mathrm{K}=1+\frac{1}{\mathrm{kT} \varepsilon_{\mathrm{O}} \mathrm{E}}\left[\alpha_{\mathrm{a}} \mathrm{P}_{\mathrm{a}}+\left(\frac{\mathrm{a}}{3}+\frac{\mu^{2}}{3 \mathrm{kT}}\right) \mathrm{P}_{\mathrm{w}}\right]
\end{aligned}
$$

The above derivation has been made in order to indicate the origin of the formula which is used for the dielectric constant of air $(9, \mathrm{p} .55):$

$$
K=1+10^{-6}\left[\frac{152}{T}\left(p+\frac{4800 \mathrm{e}}{\mathrm{T}}\right)\right]
$$

in which $\mathrm{p}$ is the atmospheric pressure and $\mathrm{e}$ is the vapor pressure of water, 1133 millibar and 18 millibar, respectively, and $\mathrm{T}$ equals $300^{\circ} \mathrm{C}$. Equation (2-24) implies a refractive index of

$$
\mathrm{n}=1+10^{-6}\left[\frac{76}{\mathrm{~T}}\left(\mathrm{p}+\frac{4800 \mathrm{e}}{\mathrm{T}}\right)\right]
$$

\section{D。 PROPAGATION THEORY RESULTS}

The results of equations $(2-5)$ and $(2-6)$ have their origin in the energy considerations discussed before. When $r$ is taken smaller or equal to the diameter or the dimension of the smallest eddies beyond which no new eddies are generated because viscosity in these consumes the energy, then the velocity difference can be expanded as a power series in $r$, of which only the first term needs to be retained. The structure function is the averaged square of that term with a proportionality constant $\left(D_{n}(r)=a_{n} r^{2} ; D_{T}(r)=a_{T} r^{2}\right.$.

The structure function (9), which is plotted in Figure 1, shows a 


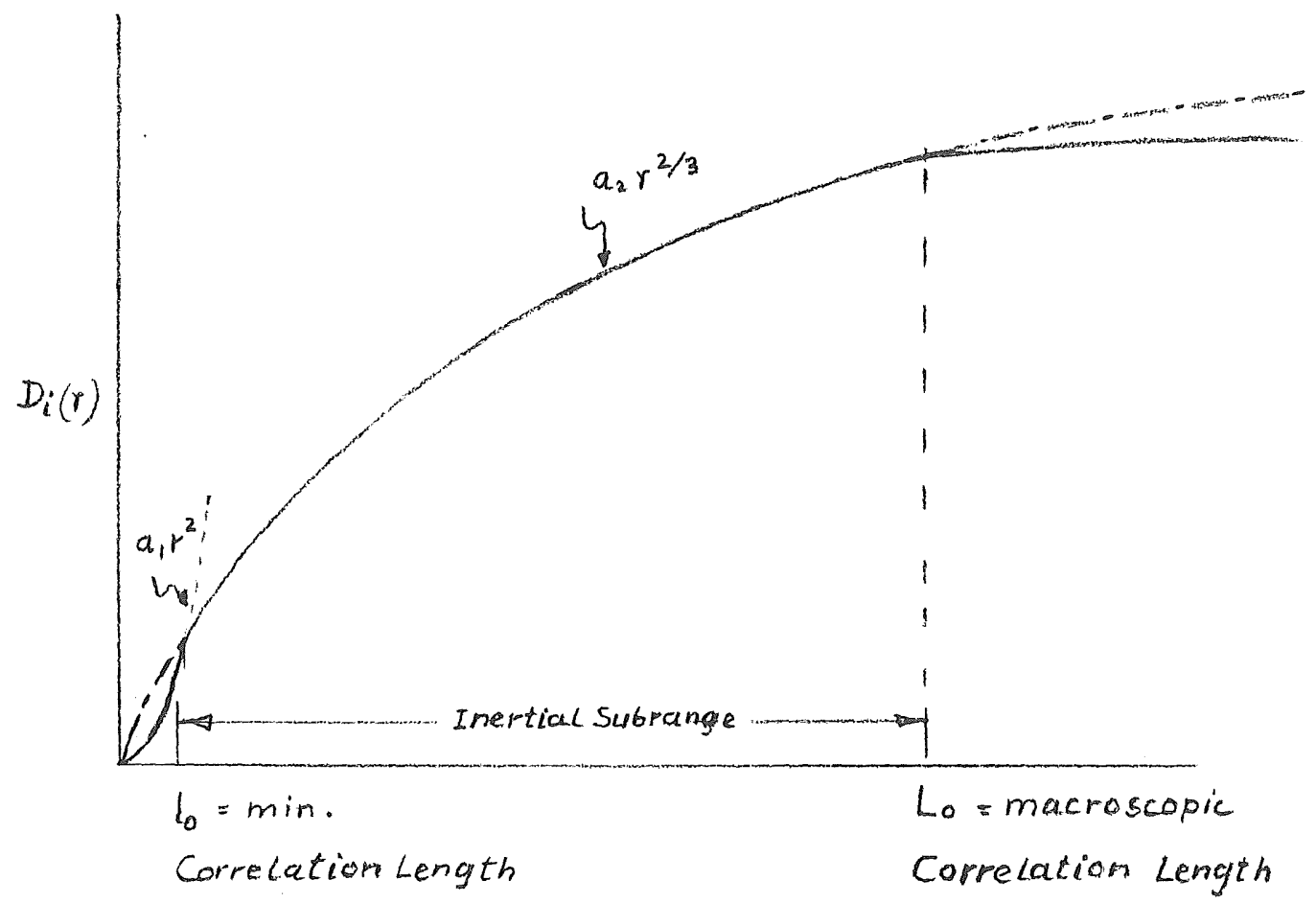

Eigure 1. Structure functions versus sedaration. 
parabolic increase in the region of $r$ near zero. The curve then continues increasing according to the $2 / 3$ power of $r$. The intersection of the two curves (extrapolated) defines the minimum correlation length $1_{0}$. As $r$ increases into the region where turbulence can no longer be considered homogeneous and isotropic, further anomalies of the curve are expected.

From the perturbation solution of the wave equation for a random medium, assuming smooth perturbations (Rytov method), the variance of scintillation log-amplitude is

$$
(\log A-\overline{\log A})^{2}=\sigma^{2}=0.124 \mathrm{C}_{n}^{2} \mathrm{k}^{7 / 6} \mathrm{~L}^{11 / 6}
$$

Equation $(2-26)$ shows that the variance is a function of wavelength $\left(k=\frac{2 \pi}{\lambda}\right)$ and of path length $(L)$ besides the refractive index structure function $\mathrm{C}_{\mathrm{n}}{ }^{2}$. To test the equation, corresponding measured values of $\sigma^{2}$ and $C_{n}{ }^{2}$ are substituted into it. In our experiments this is done at four different values of $\lambda$, namely $10.6,1.15,0.6238$ and $0.488 \mathrm{mi}-$ crons and at two values of L, 500 feet for the portable laser at 0.6238 microns and one mile at the other wavelengths.

In agreement with other researchers, our results show a saturation for values of the variance exceeding 0.6 to 0.8 (see further discussion Chapter III-H). This phenomenon is not well understood and is subject to intensive investigation. Strohbehn (19) quotes Tatarski for a modified geometric optics approach in calculating the variance of the log-amplitude fluctuations of scintillation. A relationship between the results of the modified and the classical approaches is given:

$$
\sigma_{\chi}^{2}=1-\frac{1}{\left(1+6 \sigma_{o}^{2}\right)^{1 / 6}}
$$


where $\sigma_{X}=-\langle X(L)\rangle$ (assuming that $X$ is a Gaussian random variable) is the new variance and $\sigma_{0}$ the classical. A plot of $\sigma_{\chi}$ versus $\sigma_{0}$ (Figure 2) shows that $\sigma_{\chi}$ saturates at an asymptotic limit of one. The covariance is defined as $(\log A-\overline{\log A})(\log B-\overline{\log B})$, and is predicted to fall to zero when the distance between samples $A$ and $B$ is on the order of $(\lambda L)^{1 / 2}$ (the Fresnel distance). This has been confirmed by our measurements. The spectral bandwidth of scintillation equals

$$
B_{S}=(\lambda L)^{1 / 2} / v_{t r}
$$

where $v_{t r}$ is the transverse component of the wind speed. This relates to the concept of the "frozen-in turbulence".

\section{E. RESEARCH INTO TURBULENCE PROPAGATION PHENOMENA AT THE OREGON GRADUATE CENTER}

Dr. J. Richard Kerr began research in optical propagation at the Oregon Graduate Center with a small team in 1966/67. A transmitting station was built on the roof of the West annex, which had an ideal position for propagation in different directions and lengths of up to 20 miles. A mobile receiving station, equipped with dual receivers and a two-channel processing apparatus for the computation and compilation of two-point statistical data, was temporarily located at about a mile from the transmitter.

The slightly divergent beam from a Helium-Neon Laser emerging from an expanding telescope was sampled at the receiver by two small apertures. The variance and covariance (Chapter II-D) of scintillations, spectrum, and log-amplitude probability distribution were 

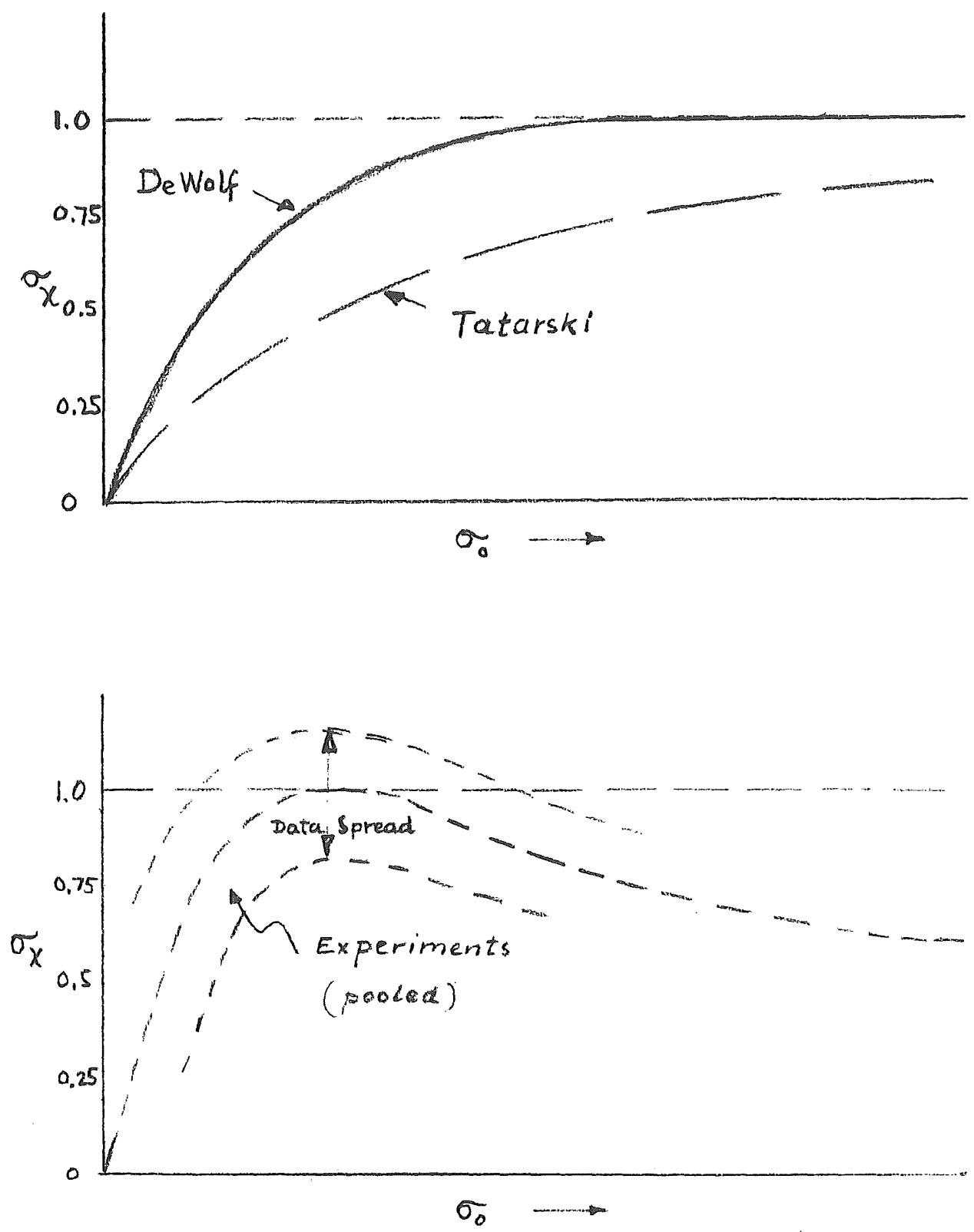

Flgure 2. Scintiliation variances. top: theoretical, bottom:pooled experimental data. 
computed in real time $(20,23)$. Transit-time dispersion and phase structure were also investigated.

At the same time, an extensive survey of the pertinent literature was made, resulting in an appraisal of the various contributions. Areas of disagreement between theory and experiment or between rival theories and areas in which little work had been reported were pointed out $(20)$.

The results of the experiments and of the survey helped in the planning of a more sophisticated experimental facility for the simultaneous investigation and real-time data processing of scintillations at three wavelengths over a long path, as well as independent correlation with turbulence data from a thermal microprobe and a portable laser at a fourth wavelength located at selected, smaller distances from the receiver. These aspects are discussed in the next Chapter. 
CHAPTER III

\section{EXPERIMENTAL FACILITY FOR THE INVESTIGATION OF TURBULENCE PROPAGATION PHENOMENA ELECTRO-OPTICAL SYSTEMS}

In this Chapter, the experimental electro-optical facility is discussed in detail, and the author's contributions described.

\section{A. THREE-WAVELENGTH PROPAGATION LINK}

The fundamental experiments on this program involve the statistics of scintillations for three coincident laser beams propagated over a long path (Figure 3). In order to obtain meaningful results in this multiwavelength experiment, which is designed to test the range of validity of statistical propagation solutions and the theoretical model of atmospheric turbulence, it is essential that the compared beams occupy the same space and be monitored simultaneously. The beams have the shape of a cone, beginning with a diameter of four centimeters at the transmitter aperture and ending 1500 meters downrange with a diameter of about one meter.

1. A stable 500 milliwatt argon ion laser operating at 488 nanometers

2. A 20 milliwatt helium-neon laser operating at 1.15 micrometers

3. A low-noise, 5-watt carbon dioxide laser operating at 10.6 micrometers.

These beams are first given identical divergences by means of 


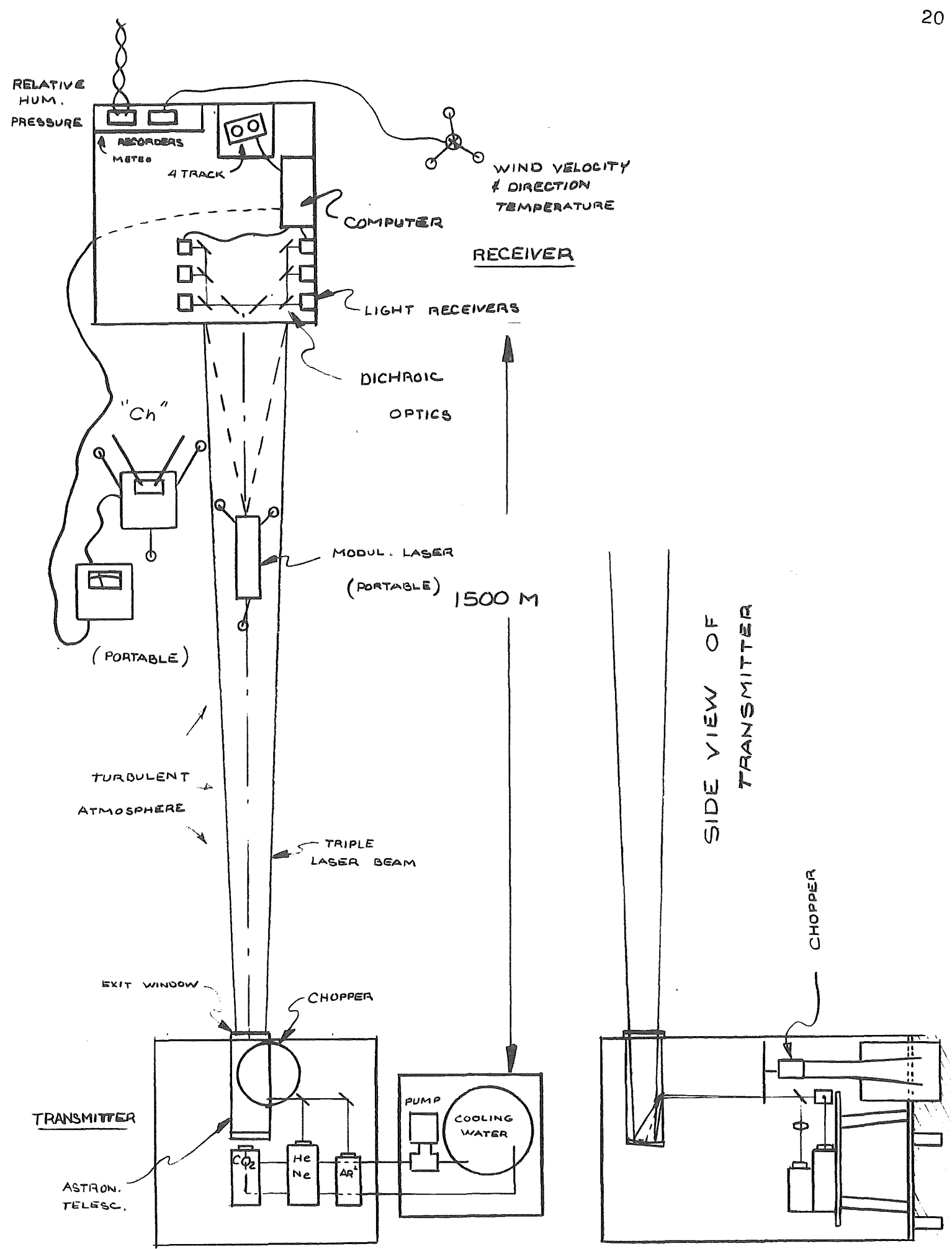

Figure 3. Oregon Graduate Center's Laser Range 
appropriate lenses; and then combined by dichroic mirrors, so that the triple beam may be chopper-modulated (at a frequency of 9 kilohertz) and finally expanded and collimated by a diffraction-limited parabolic mirror so that the described cone is obtained.

To assure that the cones are completely coincident on their milelong path, they are individually aimed. For this purpose, the system is used in reverse. A spotlight is placed at the receiver and its image at the transmitter chopping plane is observed on asbestos $(10.6$ micrometers) or phosphor paper (1.15 micrometers), while the appropriate laser beam burns a fine spot from the other side. The beams are adjusted by gymbal-mounted mirrors so that the burns coincide with the image of the spotlight. The visible laser can also be adjusted by observation at the receiver shack, which has VHF communication with the transmitter station.

The three-wavelength beam is sampled at the receiver by two apertures which can be scanned horizontally from the center of the beam towards the edges in opposite directions for variable spacing (Figure 4). Two front-surface mirrors behind the apertures deflect the beams to a system of dichroic mirrors, which separate the wavelengths and direct each into the appropriate receivers, and these transduce the light intensity into electrical intensity and preamplify the latter.

\section{B. GENERAL DESCRIPTION OF SIGNAL PROCESSING}

The electrical signals are further amplified in variable voltage gain stages, demodulated and low pass filtered before their dynamic 


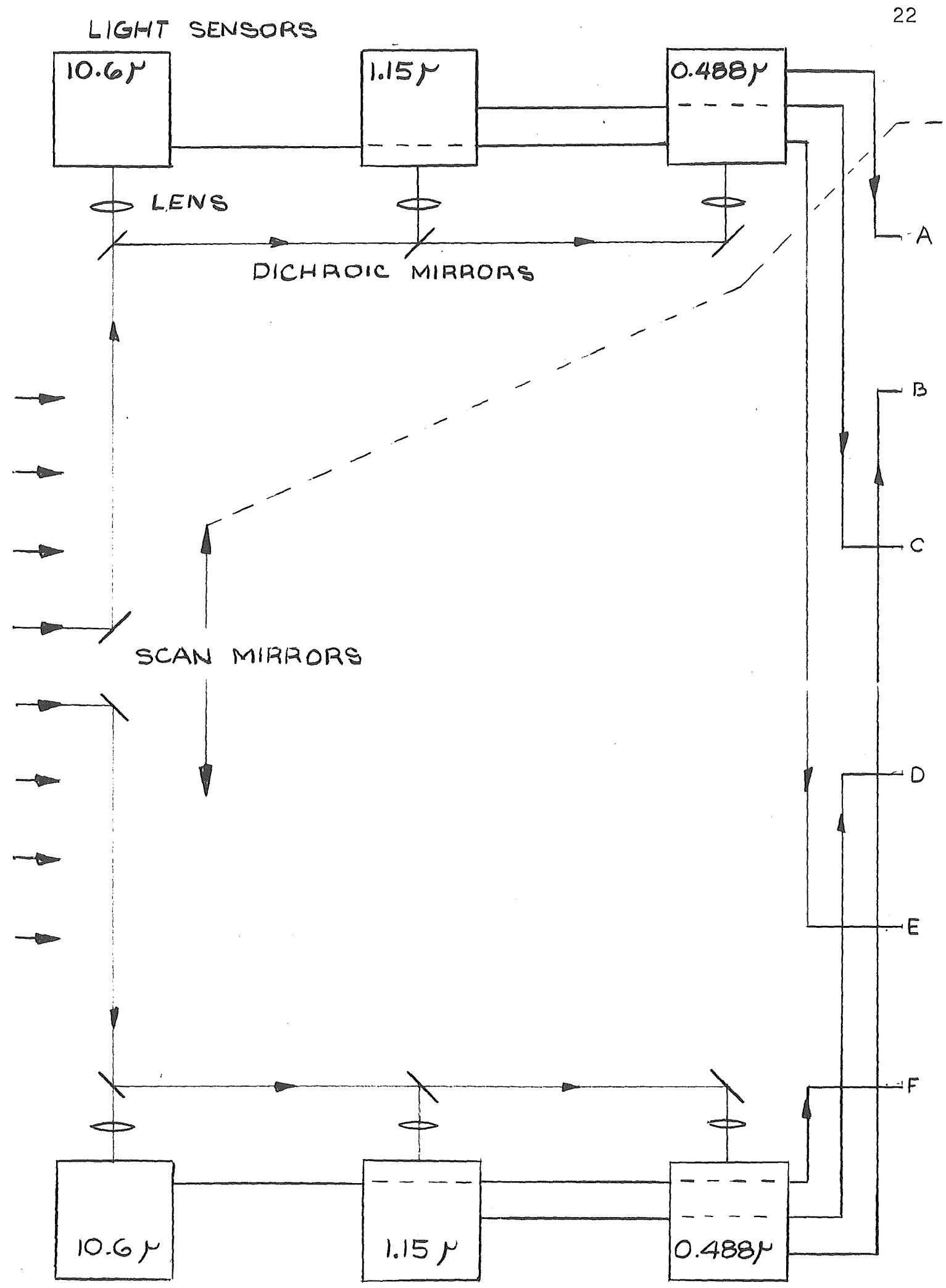

Figure 4. Optical receivers and Preamplifier 


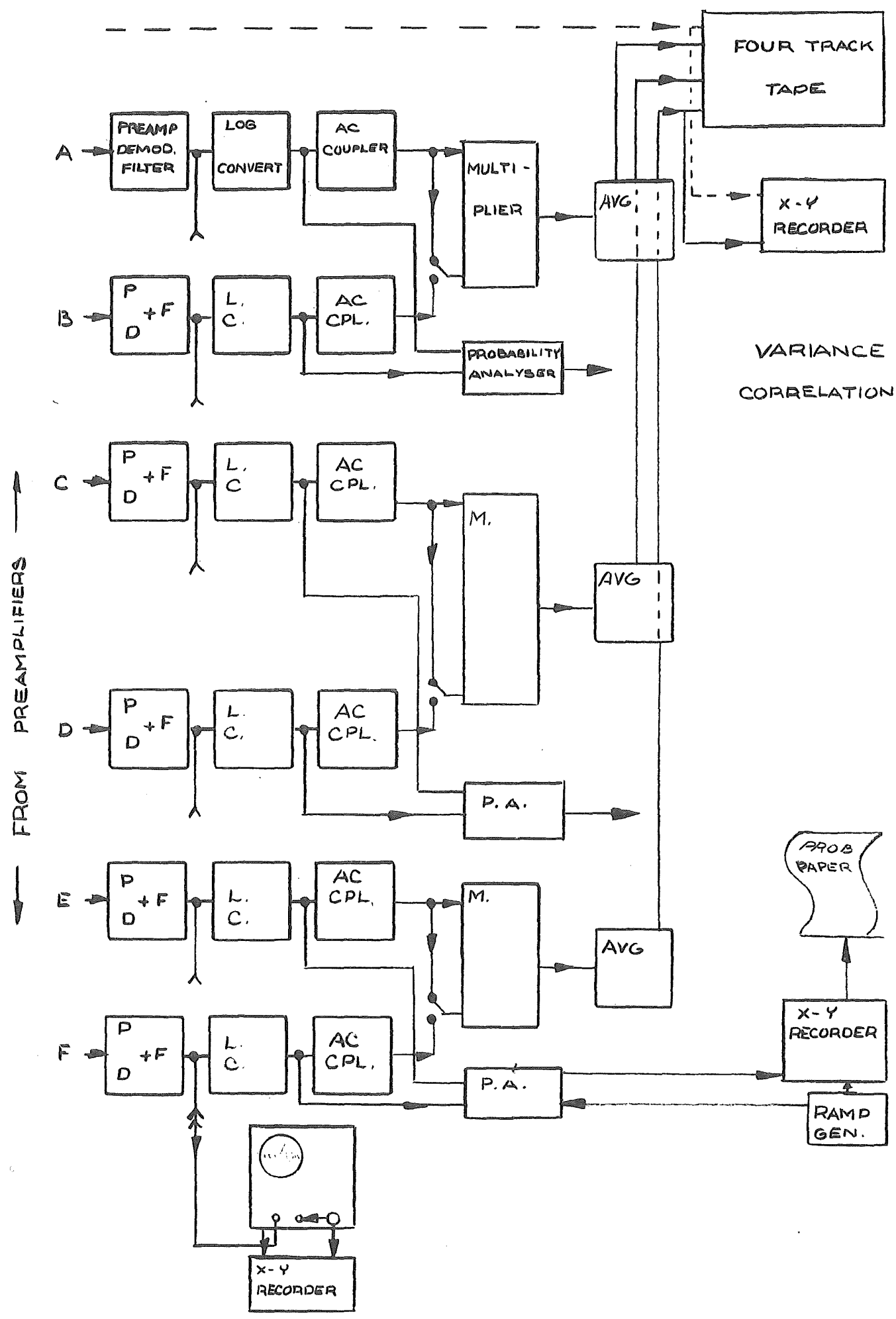

Figure 5. Computer Block Diagram 
range is logarithmically reduced. At this stage each of the six channels may be connected to a spectrum analyzer. The spectra are $\mathrm{X}-\mathrm{Y}$ recorded. The logarithmic potentials yield the log-amplitude probability distribution to three permanently connected probability analyzers. AC coupling the logarithmic fluctuations removes the average of each logarithm. The resulting fluctuations $(\log A-\overline{\log A})$ of the $\log$ amp 1 itudes from their averages are multiplied in corresponding pairs and the resulting products are averaged, yielding the crosscorrelations of scintillation as a function of wavelength and aperture separation. A four-track magnetic tape machine records the separation or comments on one track and the three simultaneous correlations for later analysis. In the laboratory the correlations are $\mathrm{X}-\mathrm{Y}$ recorded. Likewise, tapes with simultaneous probability distributions (the reference ramp on the fourth track) are transcribed in the laboratory, first from the tape onto an $X-Y$ plot, and then by hand onto Gaussian probability paper, where a log-normal scintillation amplitude results in a straight line. The described operations are shown in Figure 5.

\section{DETECTORS AND PREAMPLIFIERS}

The detectors for the visible and the near-infrared light are operated at room temperature, while those for the far-infrared radiation (mercury-doped germanium photoconductors) must be operated at liquid-helium temperature. In order to obtain linearity over a very large dynamic range, special techniques are required. The detectors are AC coupled into a virtual short circuit (summing junction of operationa1 amplifier) so that all of the incremental photo-current 
develops a voltage across the feedback resistance, regardless of the light-sensitive internal impedance of the detectors. The low-noise preamplifiers are given a wideband response, centered at the 9 kilohertz chopping frequency, and flat between 8 and 10 kilohertz.

Figure 6 shows the low-noise preamplifier used for the two infrared transducers. A voltage drop, varying according to the impinging radiation intensity, is generated by the bias current of about 2 microamperes. The low time constant of the mercury-doped germanium photoconductor, in the order of nanoseconds, assures that the 9 kilohertz modulation suffers no attenuation and excellent operation is obtained for received power ranging from 5 nanowatts to 50 microwatts, thus achieving an $80 \mathrm{~dB}$ dynamic range.

The near-infrared photodetectors are reverse-biased germanium diodes. Photons striking these units generate electron hole pairs, thus causing an increase from the dark-current level $I_{S}$. The sensitivity of the near-infrared detectors is 2 nanowatts. However, the smallest "point" apertures used are 3 millimeters in diameter, so that the power entering into the detector units is $(0.003)^{2}$ times the total transmitted power (assuming uniform intensity across the entire beam). For the near-infrared, this is 20 milliwatts, of which on $1 \mathrm{y} 180$ nanowatts pass the apertures, so that at this wavelength, the dynamic range is laser power limited to approximately $60 \mathrm{~dB}$.

The light receivers for the visible wavelength, 488 nanometers, are equipped with state-of-the-art silicon photodiodes. Their much higher dark-resistance enables the use of higher voltage gain in the first stage of the preamplifier, shown in Figure 7. A sensitivity of 


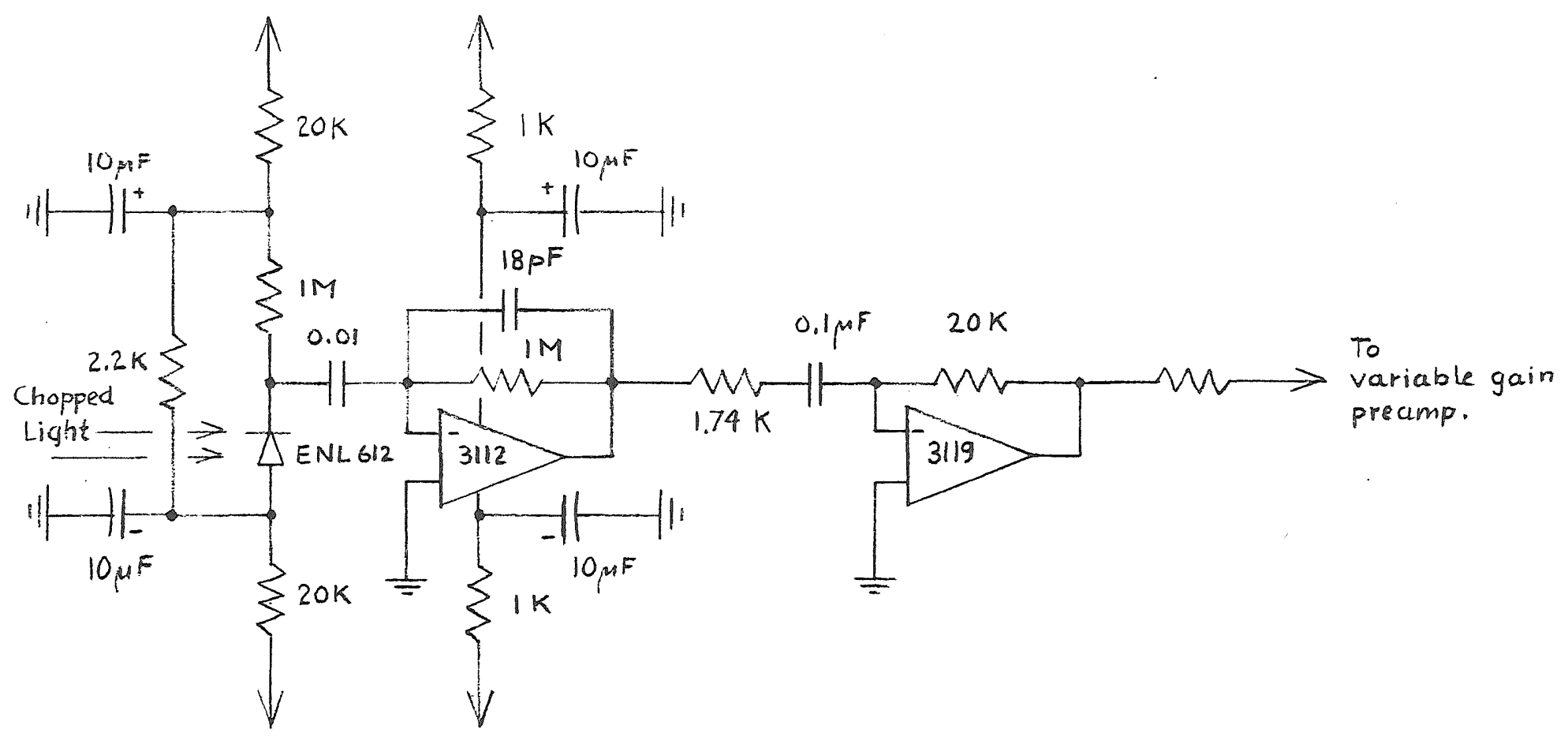

Figure 6. Light receiver and preamplifier, IR 

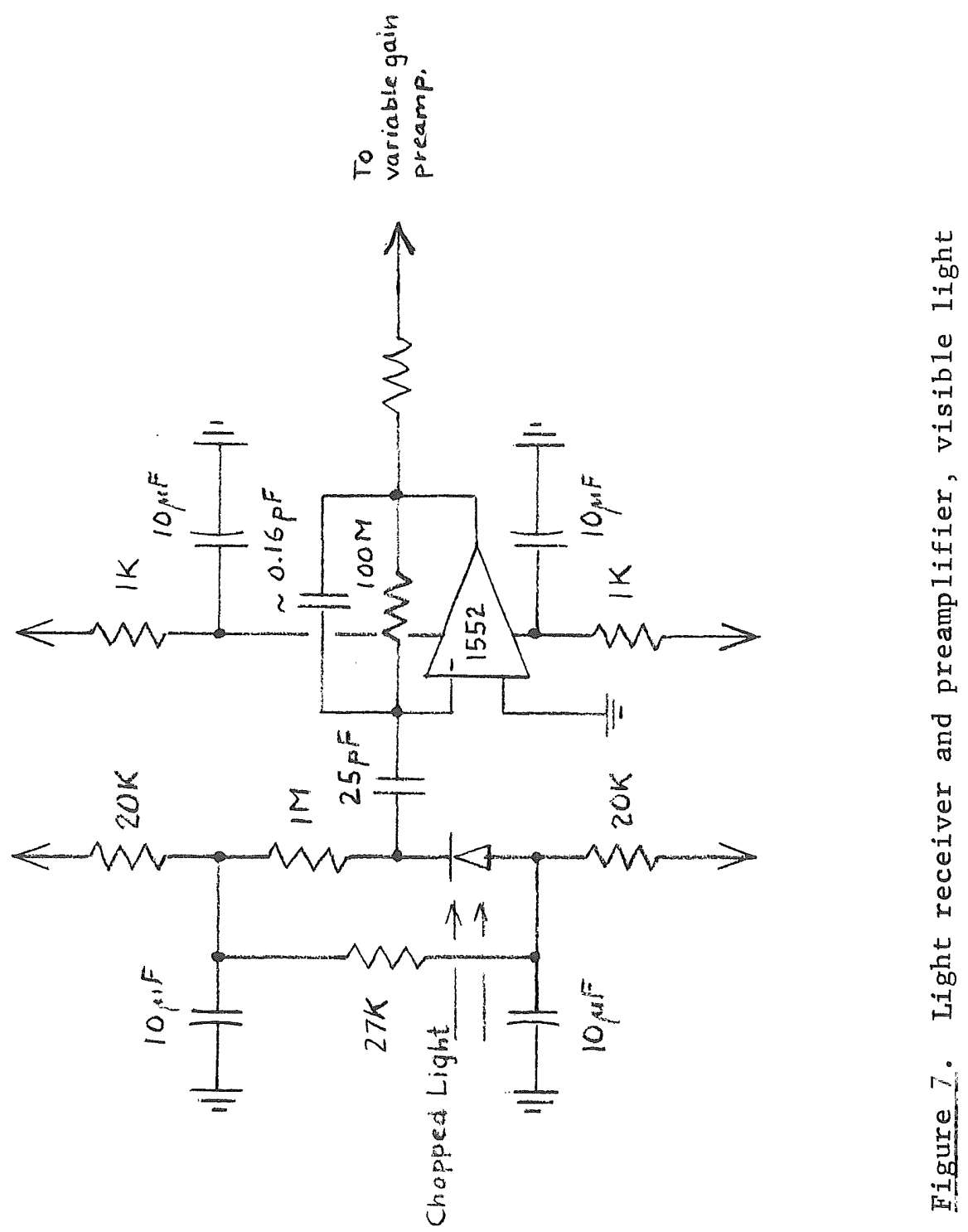
6 picowatts was achieved with these units in the laboratory, and the received laser output is more than adequate for an $80 \mathrm{~dB}$ dynamic range.

\section{PRECISION DEMODULATOR}

The three pairs of light or radiation receivers supply their output signals to six identical units providing manual level control, carrier envelope demodulation, and filtering (see Figure 8). Since turbulence is very much dependent upon the wind, weather, season and even time of day, a $40 \mathrm{~dB}$ gain adjustability assures optimal use of the available signals.

The rectification of modulated carriers by means of conventional diode circuits imposes serious limitations on the dynamic range of the signal amplitude. However, if the diode is used only to distinguish between the two directions of current flow, while a feedback amplifier circuit assumes responsibility for a linear relationship between input current and output voltage, much wider dynamic ranges can be handled $(24,25,26)$. The results which can be achieved depend on the circuit, its elements and the stability with time and temperature of the operational amplifier.

In its simplest form the circuit comprises an operational amplifier with an input resistor $R$, which determines the input current delivered by a source of $\mathrm{E}$ volts; a pair of diodes, providing distinct feedback paths for the two current directions; and an output resistor in series with one of the diodes, across which the output voltage is developed. The circuit of a demodulator with a dynamic range of more than $80 \mathrm{~dB}$, which has been realized by the author, is shown in Figure 8 . 


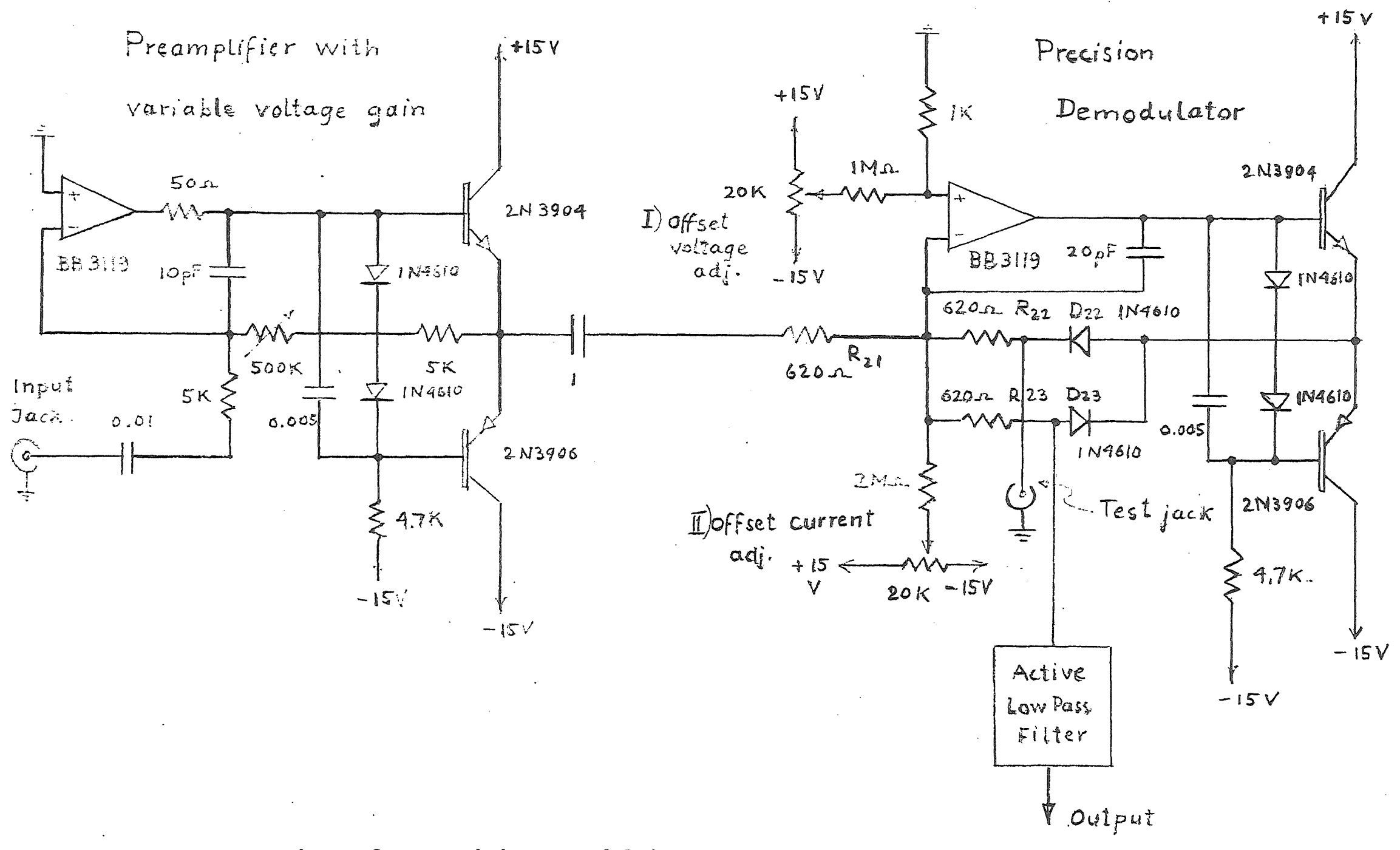

Figure 8. Precision Demodulator 
The operation is as follows: when current enters the input terminal of the amplifier through the input resistor $R_{21}$, the potential of the terminal is raised a small amount (like that of a leaky capacitor upon current influx). Consequently, the output voltage of the amplifier increases $(-K)$ times that amount, forward biases one of the diodes $\left(D_{23}\right)$, and produces a current out of the input terminal which is almost equal to the input current. (The less current the amplifier input requires to maintain the small voltage change at the input terminal, the better the equality between output current and input current.) This current traverses the output resistor $R_{23}$ in series with the diode, and an output voltage is produced.

The magnitude of this voltage with respect to ground equals the sum of the input terminal voltage $(\varepsilon)$ plus the voltage drop produced in the resistor by the output current:

$$
V_{0}=-E+\frac{(1+K) \varepsilon R}{R_{d}+R}=\frac{e_{0}}{K}\left[1-\frac{(1+K) R}{R_{d}+R}\right]
$$

where $R_{d}$ is the diode resistance, which depends on the output current $\left(I_{d}\right)$ through the diode:

$$
I_{d}=I_{s} \quad[\exp (q V / k T)-1]
$$

where

$$
\begin{aligned}
& \mathrm{k}=\text { Boltzmann's constant } \\
& \mathrm{T}=\text { temperature } \\
& \mathrm{q}=\text { electronic charge } \\
& \mathrm{I}_{\mathrm{S}}=\text { diode saturation current }
\end{aligned}
$$


Figures 9 and 10 show families of $V / I_{d}$ curves for two diodes $\left(I_{s}=2.8 \mathrm{nA}_{,} I_{s}=1.5 \mathrm{pA}\right)$. The static diode resistance is found by solving equation (3-2) for $V$ and dividing by $I_{d}$. If the diode current is represented as a multiple of $I_{s}$, such that $\left(I_{d}-p I_{s}\right)$, we have

$$
R_{d}=\frac{k T}{q} \frac{\ln (p+1)}{p I_{s}}
$$

From equation (3-1) it can be seen that for very large values of $R_{d}$, i.e., for small values of $I_{s}$ and $p$, the output voltage can become zero and even approach $-\varepsilon$, the input terminal voltage. In this case, the output voltage is grossly in error and no longer useful. The end of the useful range is defined by an output voltage error no greater than, e.g., 30 percent. This percentage is made up of leakage current, a drop in the system gain, and capacitive bypass.

When the input current further decreases and finally reverses its direction, the static resistance of the previously conducting diode $\left(D_{23}\right)$ exceeds that of the diode which has just been forward biased $\left(\mathrm{D}_{22}\right)$. This diode limits the potential across the demodulating diode $\left(D_{23}\right)$ and, thus, minimizes reverse leakage current through it. Even at moderate carrier frequencies $(10,000 \mathrm{~Hz})$ the diode capacity must be taken into account, because of the high value of dynamic (average) resistance which the diodes can assume at low current. A calculation of the maximum admissible saturation current for the diodes is made in Appendix C. The calculation assumes a system optimized by balancing the causes of error against each other. Each cause is allowed to contribute the same percentage of error. Since it is not 

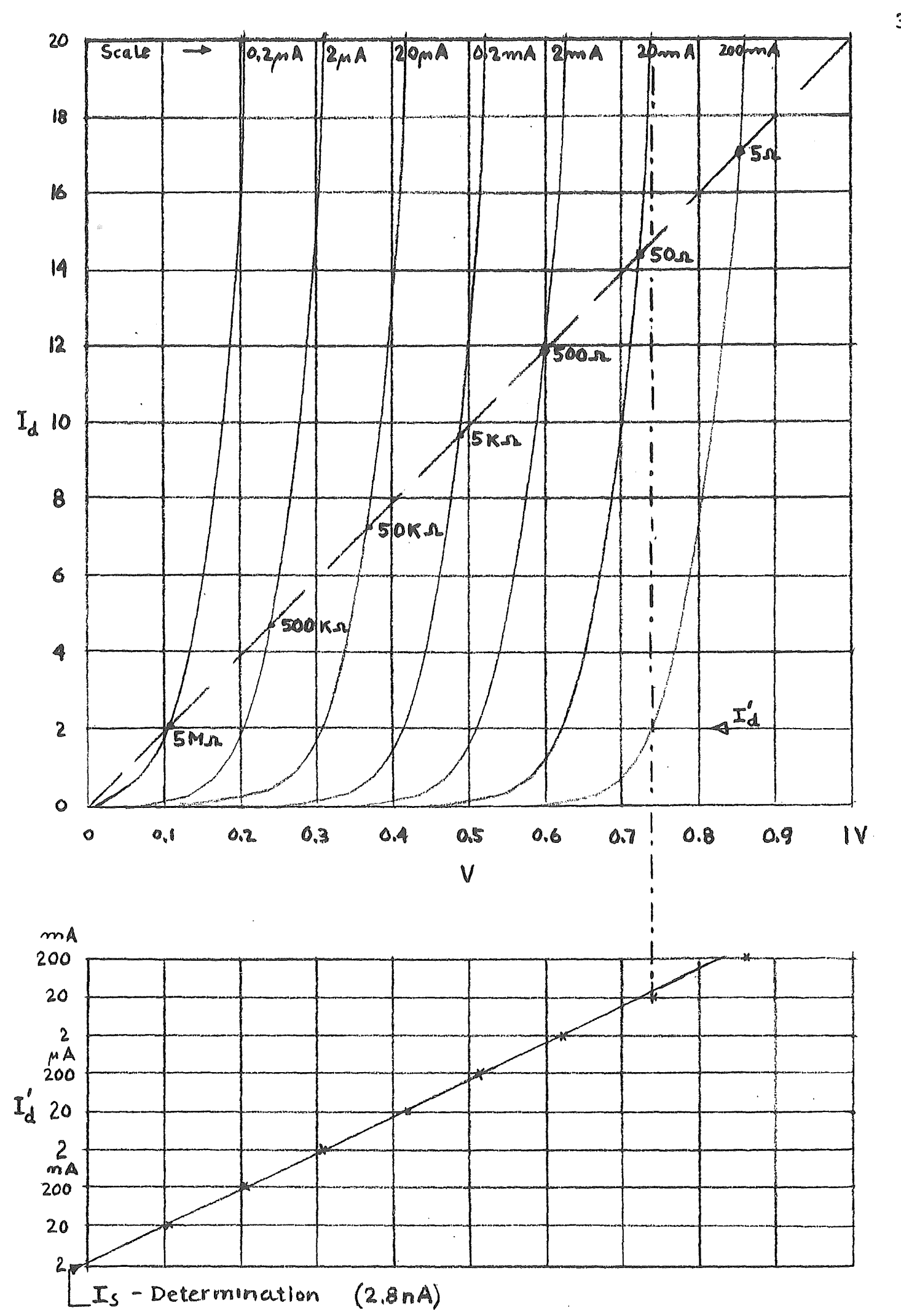

Figure 9: Diode current vs voltage characteristics, medium saturation current $I_{s}$. 

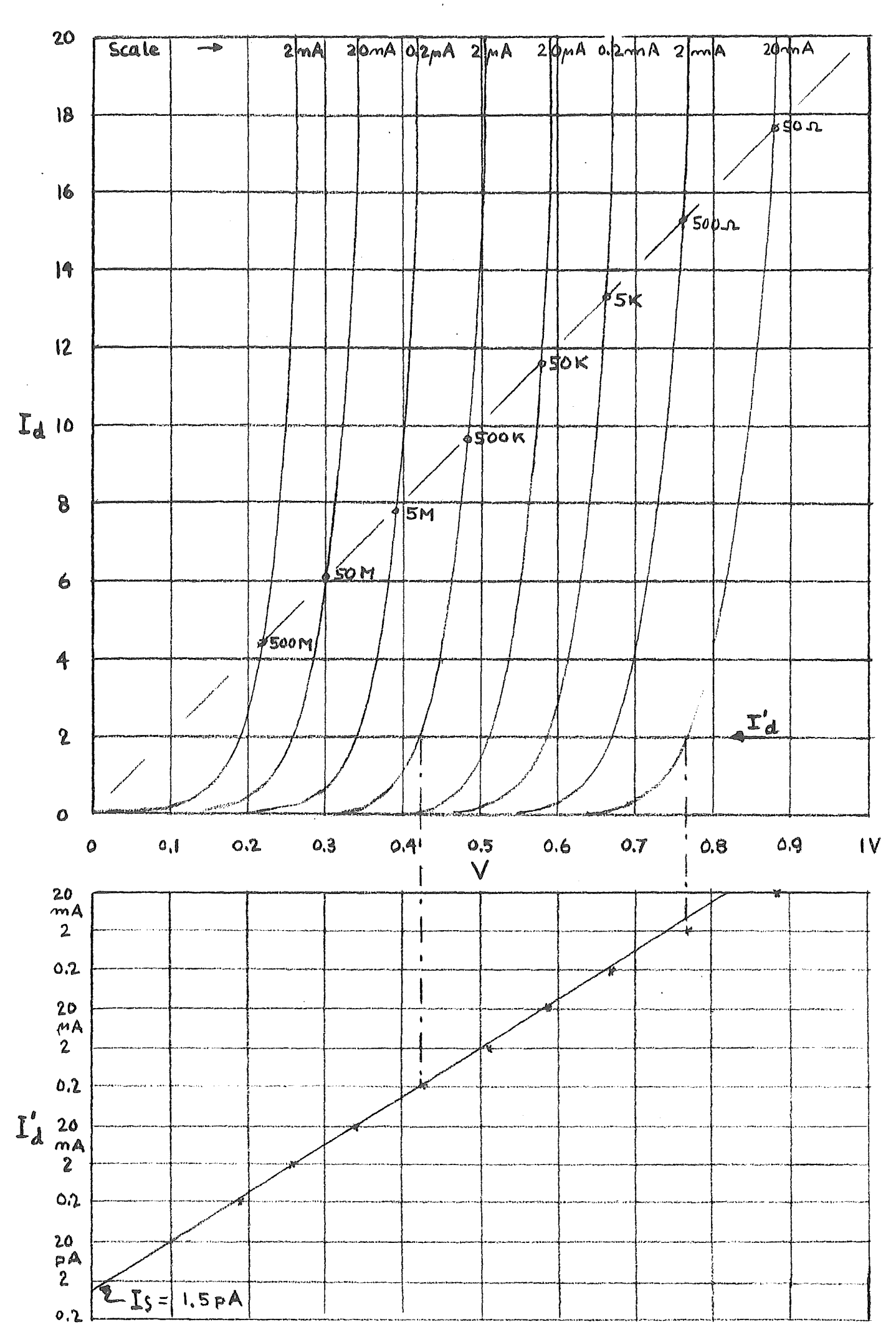

Figure 10. Diode current vs voltage characteristics, 10w satu= ration current Is 
easy to produce operational amplifiers which have negligible offset voltage, a further assumption is that the amplifier offset voltage has been cancelled. This is achieved by biasing the non-inverting or reference input terminal, so that when the inverting terminal is at ground potential, the output terminal is also at ground potential. This condition must hold when the input current is zero. Since the voltage difference between the amplifier input terminals requires a small offset current to maintain this condition, the offset is supplied from an adjustable current source into the inverting terminal.

In the endeavor to make precise adjustments, it has been found extremely helpful to introduce a resistor $\left(R_{22}\right)$ equal in value to the output resistor $\left(R_{23}\right)$, in series with the second diode $\left(D_{22}\right)$. The electrical symmetry now merely demands that the output signals from both resistors be equal and opposite in polarity, to guarantee that the adjustment is optimal (Figure 11). Therefore, it is advantageous to limit the bandwidth of the oscilloscope vertical amplifier (Tektronix Type 1A7). Demodulator output voltage oscillograms are shown in Figure 12; (a) shows the demodulator output due to a signal obtained by mixing two sine waves of neighboring frequencies; (b) and (c) show demodulated sine wave signals of intermediate and minimum amplitude.

The current booster stages following the preamplifier and the demodulator amplifier provide an extension of the dynamic range by a factor of ten to a total of more than 80-90 dB. Figure 13 shows three pairs of input and output voltages of the demodulator, at high, medium and low amplitude. The sequence of (negative) half-waves from the demodulator is smoothed in a five-pole, low-pass Bessel filter with a 

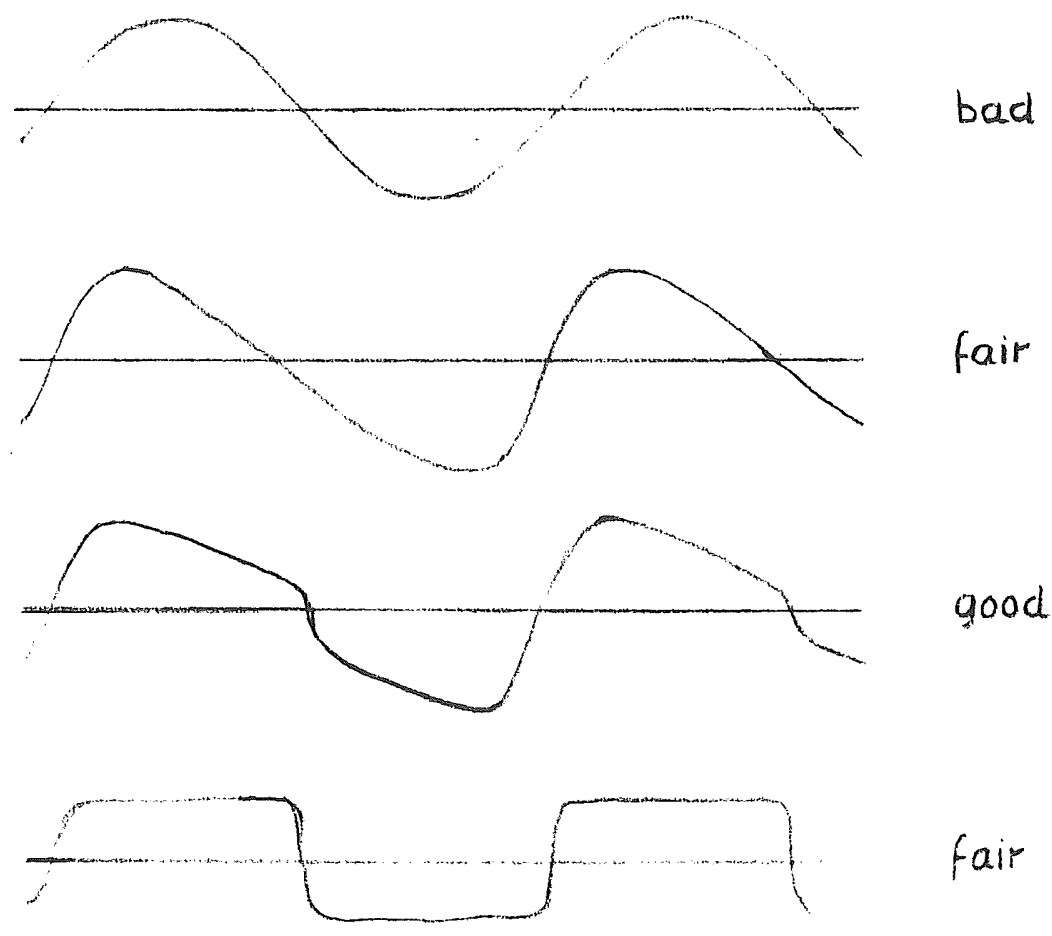

bad

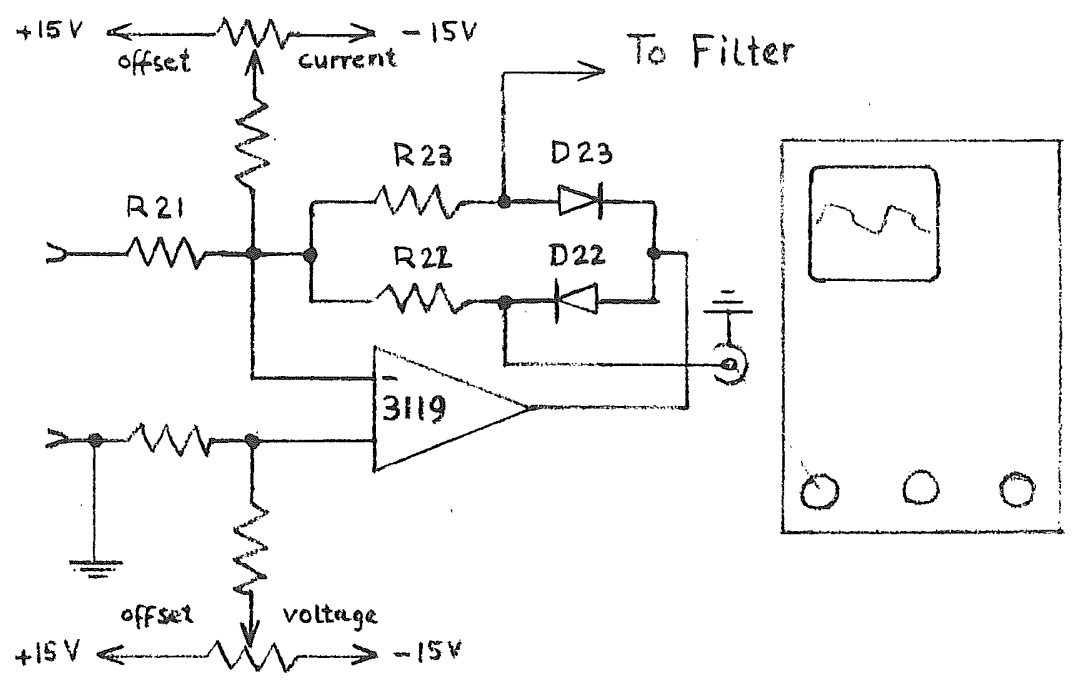

Figure 11. Precision Demodulator symmetry adjustment. 


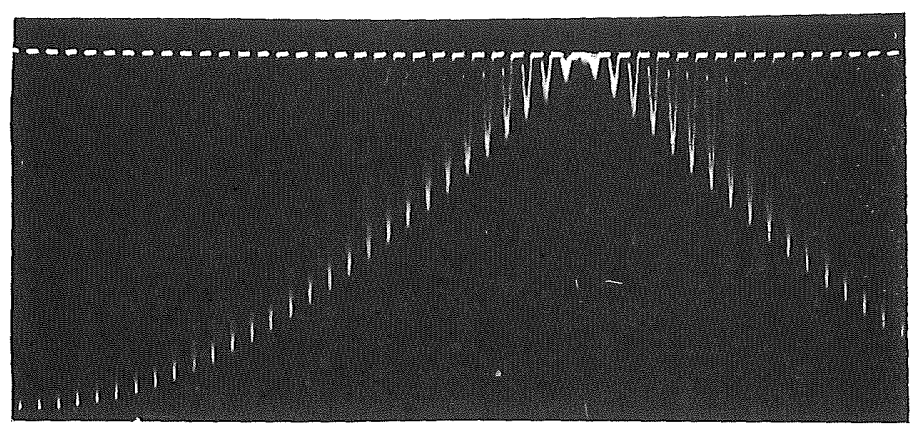

Sinewave modulated; vertical: $1 \mathrm{~V} / \mathrm{cm}$

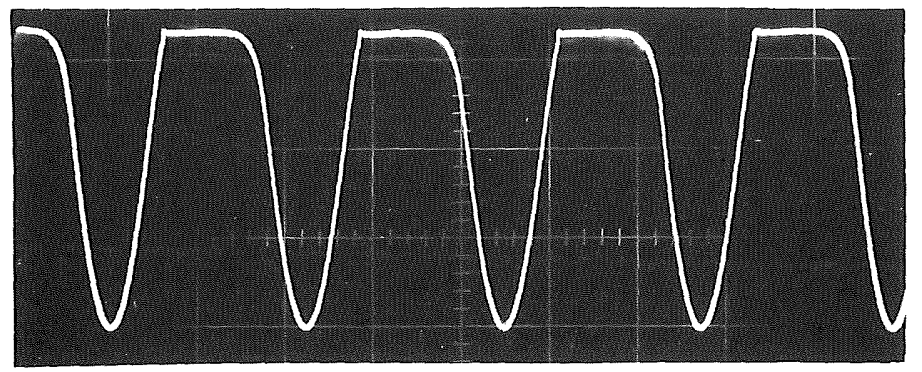

Input: $0.3 \mathrm{Volts}$; vertical: $0.2 \mathrm{~V} / \mathrm{cm}$ :

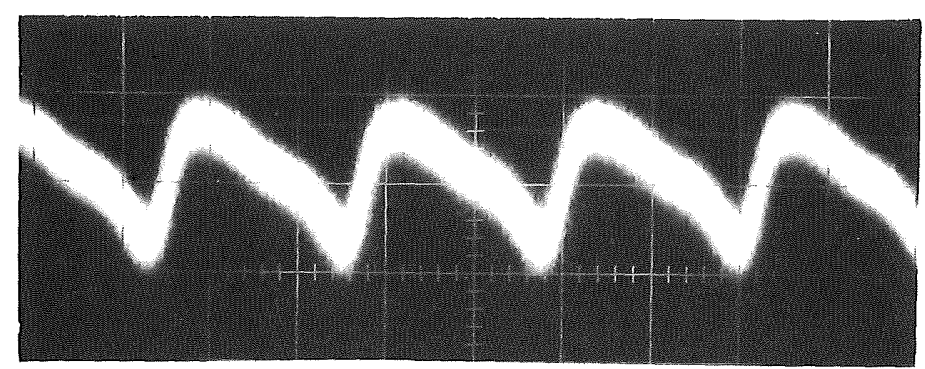

Input: $30 \mathrm{microvolts}$; vert॰: $0.2 \mathrm{mv} / \mathrm{cm}$;

Flgure 12. Demodulator, Test jack output voltage. Vertical bandw deh: 100-30 000Hz. Slgnal frequency: $9 \mathrm{kHz}$. 

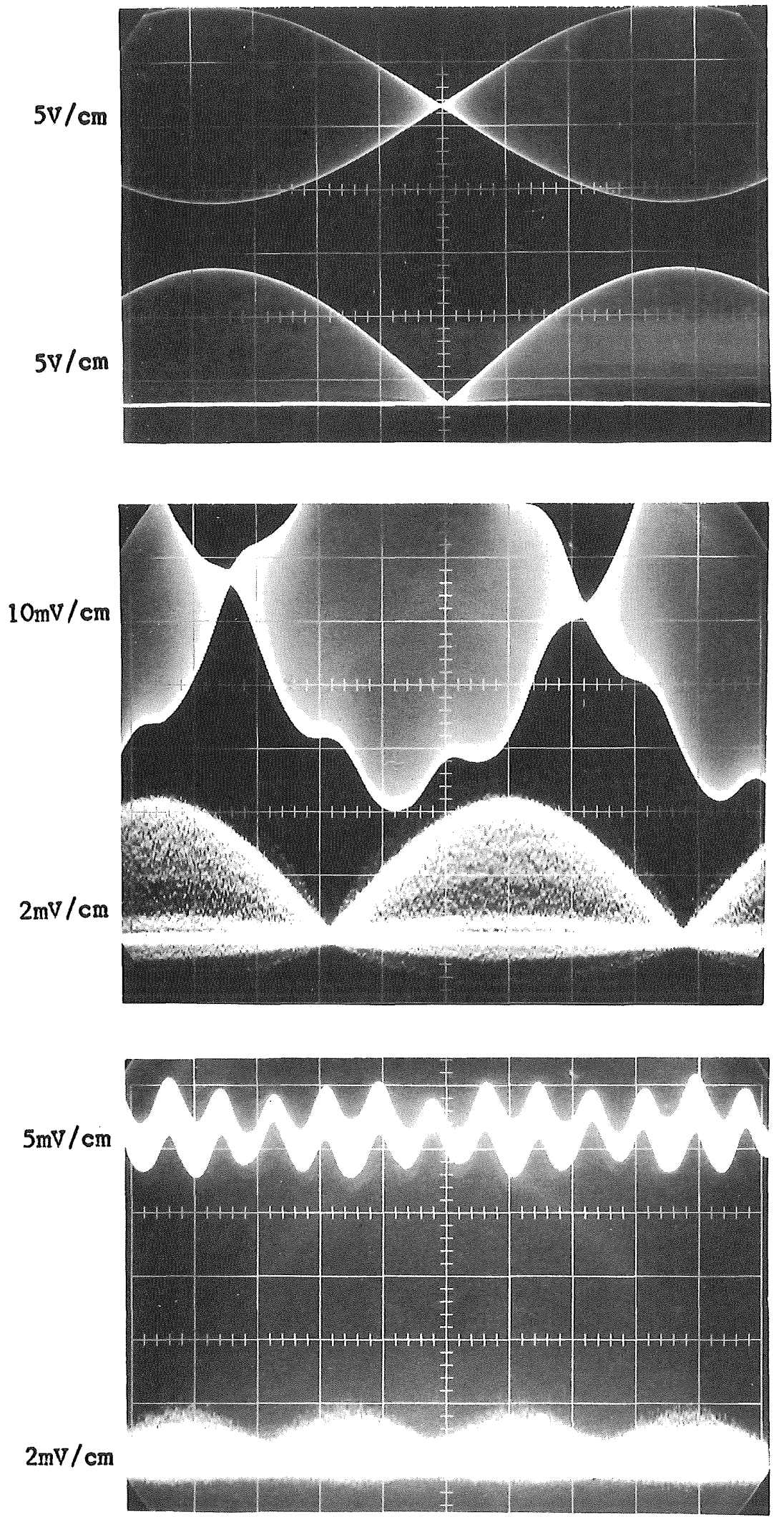

Figure 13. Input and output voltages of precision demodulator. Horl zontal sensitivities: $10 \mathrm{~ms} / \mathrm{cm}$, bottom $20 \mathrm{~ms} / \mathrm{cm}$. 
cutoff at $2 \mathrm{kHz}$. This filter has high input impedance and low output impedance, and thus, only slightly loads the demodulator, while presenting a voltage source to the following stage. Since this unit is often a logarithmic converter, as in our case, for instance, the filter's linear phase response and attendant freedom from overshoot is necessary, if large errors in the logarithmic output cannot be tolerated.

\section{E. ANALOG COMPUTER}

The demodulated and filtered signals now enter the analog computer. These identical parts, one for each wavelength, are housed in a closed aluminum cabinet. The inside temperature is held at $35^{\circ}$ Centigrade by feedback control of the current through a heater element. The air is blown through the heater and circulated in the cabinet in order to reduce thermal gradients. In this way, the non-1inear computing elements are maintained at their carefully trimmed operating points .

Figure 5 shows within the dash-dotted line the electronic units contained in the cabinet. A logarithmic conversion is the first step in all six channels. This conversion compresses the wide signal range and gives equal range to equal ratios at low and high levels. It conveniently linearizes an exponential phenomenon of nature: the logarithm of the scintillation amplitudes is predicted to have a Gaussian probability density function. The log units are described in the following section.

In Figure 5, the. signals from the $\log$ units are seen to be $\mathrm{AC}$ coupled to voltage followers. Thereafter, either one channel can be 
connected by a switch to both inputs of a multiplier, resulting in the log amplitude variance; or the two channels supply the factors of the product, resulting in the covariance. The probability distribution of the logarithm is obtained in commercial units which have been modified for near-base-band to $1 \mathrm{kHz}$ response. The simultaneous threewavelength variances, covariances and probability distributions are recorded on a four-track FM tape recorder, for later laboratory processing. The fourth track is used in each case for comments; to record the sampling mirror separation for the covariance or the reference ramp voltage (probability). Because of vibrations in the translating mirror drive, discrete sampling aperture distances are used.

The linear amplitude spectra are obtained by playing a tape recording of the demodulator-filter outputs into a Tektronix 1L5 Audio Frequency Spectrum Analyzer. In order to obtain better averaging, the slowest sawtooth from the time base of a Tektronix Type 547 Main Frame was further reduced by a factor of ten. The sawtooth and vertical output are also connected to a Hewlett-Packard X-Y Recorder to produce accurate, permanent records of the spectrum.

In the case of the probability distribution, an $X-Y$ recording is made directly from the probability signal and the sawtooth on the tape. This $\mathrm{X}-\mathrm{Y}$ plot is then hand-smoothed and transferred to probability paper, where a Gaussian distribution results in a straight line whose slope gives the variance of scintillation.

\section{F。 PRECISION LOGARITHMIC AMPLIFIERS}

The commercial logarithmic units obtained for this project were 
found to be insufficiently stable and not accurate enough for the purpose. Hence, the author designed alternate circuits for this function (Figure 14)。

The logarithmic conversion relies on the following facts:

An operational amplifier with a negative feedback path forces all currents. entering the summing node from the input out through the feedback path so as to minimize the current and the voltage $(\varepsilon$ ) between the summing node and the reference node (the input terminals of the operational amplifier). The current into the inverting terminal node of an operational amplifier is:

$$
\frac{\varepsilon-\mathrm{e}_{\mathrm{o}}}{\mathrm{Z}_{\mathrm{f}}}+\frac{\varepsilon-E}{\mathrm{R}}+\frac{\varepsilon}{\mathrm{R}_{\text {in }}}=0 \quad \text { (Kirchoff's law) }
$$

where

$$
\begin{aligned}
& \varepsilon=\text { potential difference between input terminals } \\
& e_{0}=\text { operational amplifier output voltage } \\
& \mathrm{K}=\text { operational amplifier voltage gain } \\
& \mathrm{z}_{\mathrm{f}}=\mathrm{y}_{\mathrm{f}}^{-1} \text { feedback impedance } \\
& \mathrm{R}_{\text {in }}=\mathrm{y}_{\text {in }}^{-1} \text { resistance between input terminals }
\end{aligned}
$$

Substituting $(-\varepsilon K)$ for $e_{o}$ gives:

$$
\varepsilon\left[y_{f}(1+K)+y+y_{i n}\right]=E y
$$

The fraction $y /\left[y_{f}(1+K)+y+y_{i n}\right]$ is seen to be very sma11, hence $\varepsilon$ is also very smal1 and may be neglected in rewriting Kirchoff's law $(3-4)$ : 


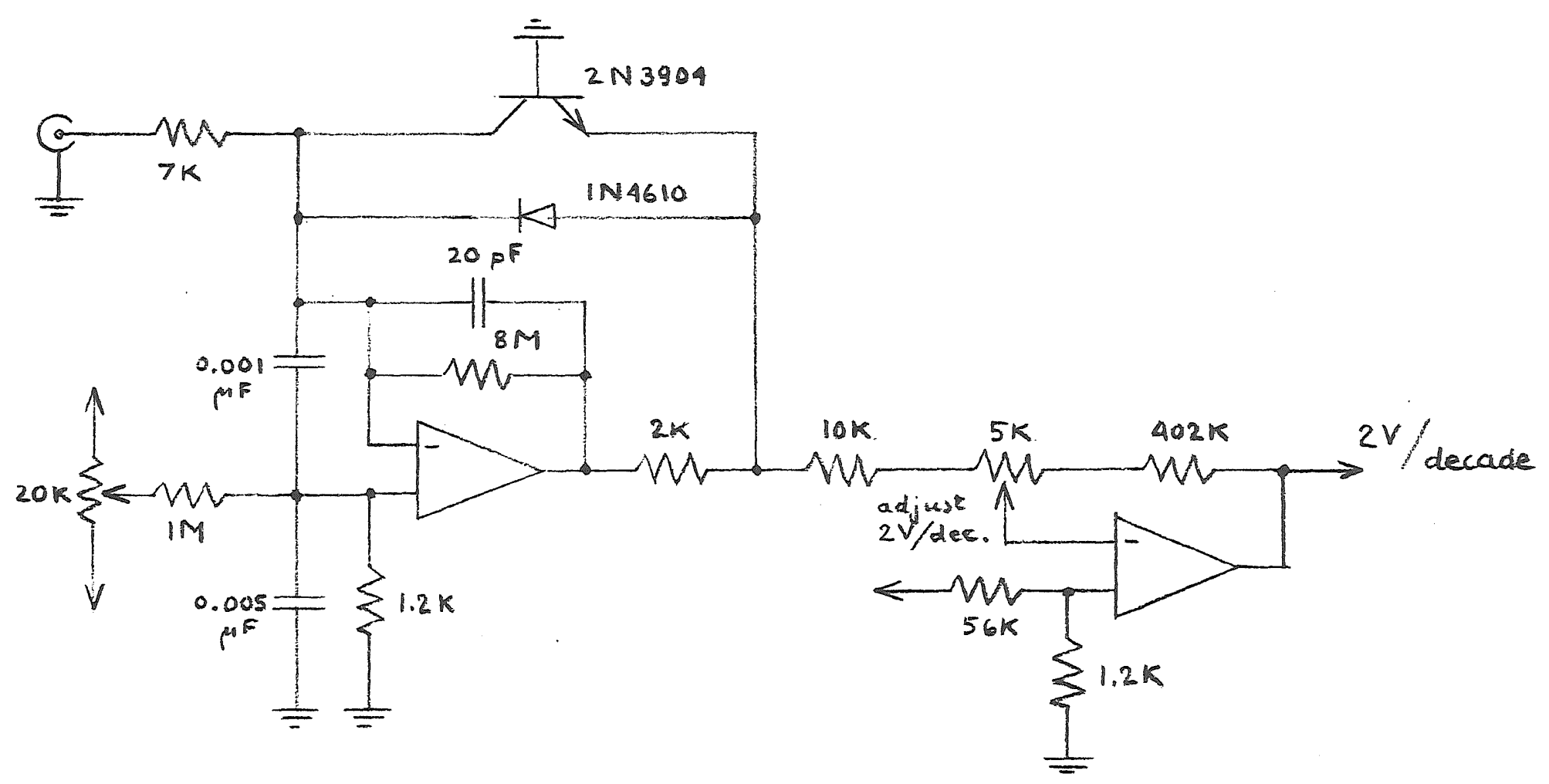

Figure 14. Iogarithmic converter and voltage amplifier 


$$
\frac{e_{O}}{z_{f}}+\frac{E}{R}=0
$$

$\mathrm{E} / \mathrm{R}$ is the input current and $\mathrm{e}_{\mathrm{O}} / \mathrm{Z}_{\mathrm{f}}$ is the feedback current.

The collector current of a transistor, with both its base and its collector terminals at the same potential, varies exponentially with the emitter-base potential $\left(\mathrm{V}_{\mathrm{e}}\right)$. The equations for the emitter and collector currents in a transistor are:

$$
\begin{aligned}
& I_{e}=I_{e s}\left[\exp \left(q V_{e} / k T\right)-1\right]-a_{r} I_{c s}\left[\exp \left(q V_{c} / k T\right)-1\right] \\
& I_{c}=-a_{f} I_{e s}\left[\exp \left(q V_{e} / k T\right)-1\right]+I_{c s}\left[\exp \left(q V_{c} / k T\right)-1\right]
\end{aligned}
$$

where

$$
\begin{aligned}
& I_{e}=\text { emitter current } \\
& I_{e s}=\text { emitter saturation current } \\
& q \quad=\text { electronic charge }=1.6 \times 10^{-19} \text { Coulombs } \\
& V_{e}=\text { emitter-base voltage } \\
& k=\text { Boltzmann's constant } 1.38 \times 10^{-23} \\
& T=\text { absolute Temperature }{ }^{\circ} \text { Kelvin } \\
& a_{r}=\text { reverse current gain } \\
& a_{f}=\text { forward current gain (very nearly unity) } \\
& I_{c s}=\text { collector saturation current } \\
& v_{c}=\text { collector-base potential }
\end{aligned}
$$

These formulae somewhat idealize the true facts by neglecting a number of small contributions from surface currents and other sources, which are, to some extent, under control in the manufacturing process. If 
the collector-base voltage $V_{c}$ is made zero, the corresponding exponential term goes to 1 , and the whole second term on the right side to zero. The factor $a_{f}$ is almost unity, to within a fraction of a percent. Although this fraction increases as the current becomes very sma11, $a_{f}$ may be taken as fairly constant.

For $\mathrm{V} \quad 5 \mathrm{kT} / \mathrm{q}=130$ millivolts, equation (3-8) may be written:

$$
I_{c}=-a_{f} I_{e s} \exp \left(q V_{e} / k T\right)
$$

Using $10=\exp (2.3)$, the potential corresponding to a current increase by a factor of ten is 60 millivolts $\left(V=2.3 \times 26 \times 10^{-3}\right)$.

A circuit which realizes the conditions required by the last equation is shown in Figure 14. The inverting terminal of an operational amplifier - forced to zero potential by negative feedback - is connected to the collector of a transistor, and the base of the transistor is connected to ground. The base current, which includes components due to surface leakage and space charge, is thus disposed of, and, to the extent that offsets of voltage and current have been compensated, the collector and the base are at the same potential as required.

The emitter current is supplied from the operational amplifier output terminal through a resistor which has the dual purpose of protecting the transistor from excessively high currents and of stabilizing the closed loop. The stability analysis given in Appendix B demonstrates the necessity of the capacitor from the output to the inverting terminal. 
A diode bypasses the transistor in the reverse direction in order to prevent its breakdown and keeps the amplifier from going into saturation. Frequency response deteriorates, especially at low currents, when the emitter resistance of the transistor becomes very high. A high value resistor, between amplifier output and collector, defines an upper bound of the time constant, compatible with the desired operation.

Proper frequency response is thus guaranteed to at least $2 \mathrm{kHz}$ and, though normal operation does not require it, the circuit could be used to perform both demodulation and logarithmic conversion.

A voltage amplifier with a gain of 33 brings the sensitivity of the logarithmic converter from $60 \mathrm{mV}$ per decade to 2.0 volts per decade. Tests at sma11 and large sinusoidal input signals (produced by the beat of the outputs from two sine wave generators operating near $9 \mathrm{kHz}$ ) were conducted. Table I and the photographs in Figure 15a and $15 \mathrm{~b}$ represent the performance of the complete system, demodulator and logarithmic converter. More than half a dozen units were built and tested, with consistent1y good results.

\section{G. PORTABLE LASER SYSTEM}

Since the scintillations seen over the one-mile path are subject to the saturation phenomenon described in Chapter II-D, it is desirable to measure the optical strength of turbulence with a short-range laser, such that the theory leading to equation (2-26) may be expected to apply. For this purpose, a $0.5 \mathrm{~mW}$ laser operating at 6328 Angstroms was equipped with a battery supply and an electronic chopper, making it 
TABLE I

LOGARITHMIC CONVERSION;

TYPICAL RESULT

\begin{tabular}{|c|c|}
\hline $\begin{array}{c}\text { Input Voltage (9 kHz) Into Demodulator } \\
\text { From Hewlett-Packard Precision Oscillator }\end{array}$ & $\begin{array}{c}\text { Output Voltage } \\
\text { Digital Voltmeter }\end{array}$ \\
\hline 3.16 Volts RMS & 9.88 Volts DC \\
1.00 & 8.88 \\
0.316 & 7.89 \\
0.100 & 6.88 \\
0.0316 & 5.87 \\
0.0100 & 4.88 \\
0.00316 & 3.87 \\
0.00100 & 2.88 \\
0.000316 & 1.90 \\
0.000100 & 1.44 \\
\hline
\end{tabular}




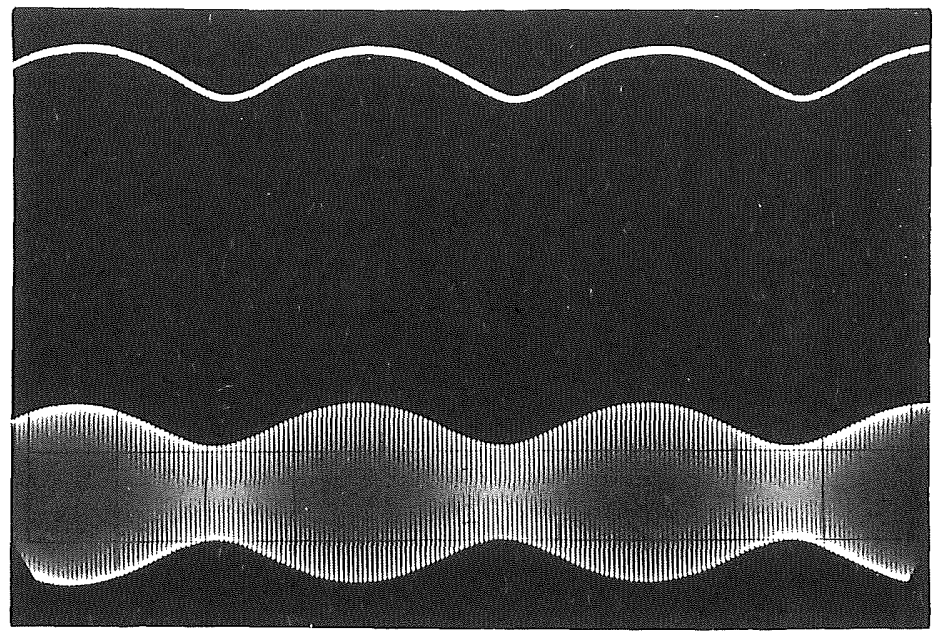

Top: Demodulated and filtered signal; $2 \mathrm{~V} / \mathrm{cm}$ Bottom: Corresponding carrier. $2 \mathrm{~V} / \mathrm{cm}$

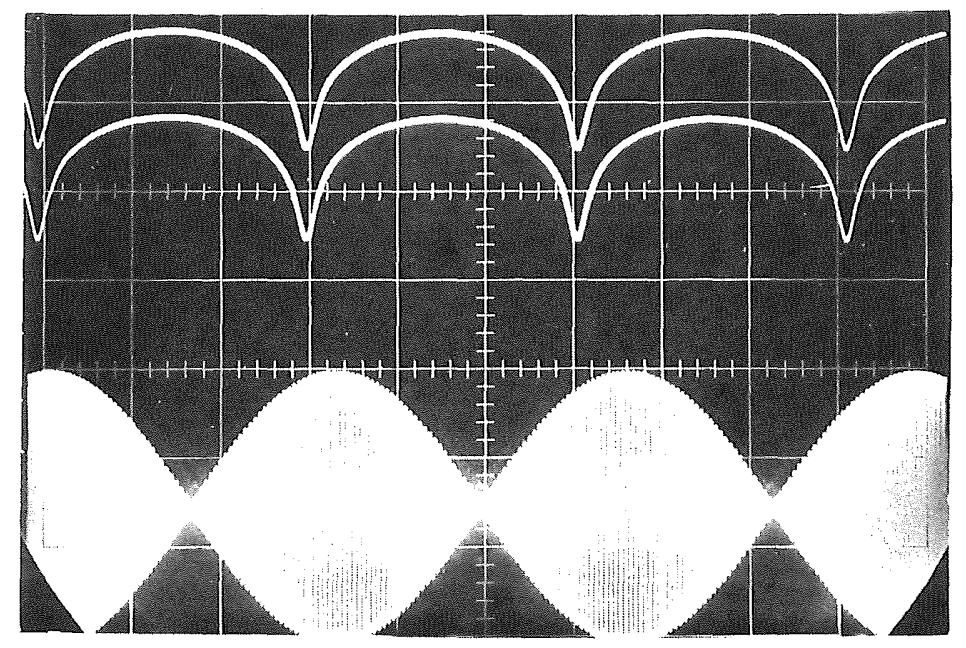

Top: Logarithm of rectified and filtered signal due to carrier shown at bottom. Both $2 \mathrm{~V} / \mathrm{cm}$

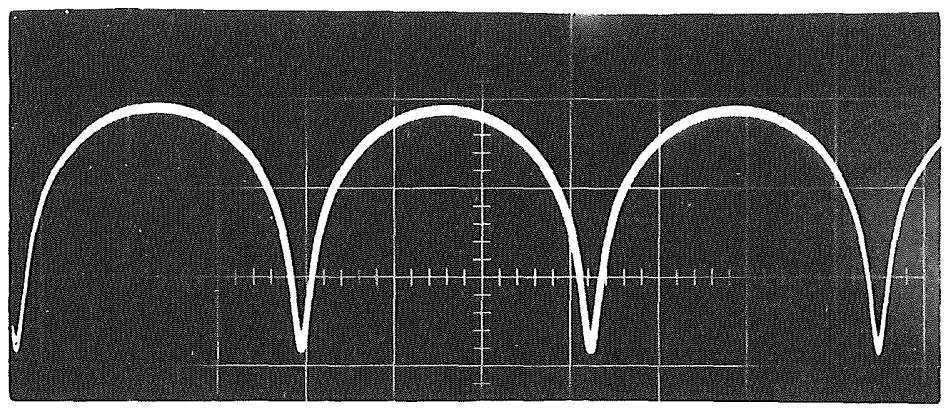

Superimposed output signals of two log-converters responding to the same input signal. $1 \mathrm{~V} / \mathrm{cm}$

Figure 15. Modulated carrier, demodulated and logarithmic signals. 
completely portable. In operation, it is supported by a sturdy tripod. The high sensitivity of the beam intensity to the discharge potential is used for modulating the laser with a 150 volt peak-to-peak square wave generator in series with the supply voltage. The generator circuit (Figure 16) is operated entirely by the current supplied to the laser through it. A 150 volt zener diode protects the circuit from overvoltage, and a 10 volt zener diode serves as reference to the voltage regulator $Q_{1}$, which guarantees a sufficiently stable operating potential for the $9 \mathrm{kHz}$ multivibrator $\mathrm{Q}_{2}, \mathrm{Q}_{3}$. The multivibrator output is DC coupled through an 11 volt zener diode, to the base of the highvoltage transistor $\mathrm{Q}_{4^{\circ}}$. The capacitors shunting the zener diodes reduce the generated diode noise. When $Q_{4}$ is turned on, the potential across the entire modulating circuit is 10.6 volts; when $Q_{4}$ is turned off, this potential jumps to the 150 volt limit. For a proper supply voltage, the 140 volt swing results in a clean 50 percent intensity modulation. Too low or too high a voltage causes spurious oscillations at much higher frequencies, superimposed on the $9 \mathrm{kHz}$ modulation. However, the adjustment is uncritical and remains stable.

\section{H. EXPERIMENTAL RESULTS - OPTICAL}

Although a detailed description of experimental results is beyond the scope of this report, sample results are given in the form of graphs accompanied by brief comments. The components and the overall system are operating as designed and the data are proving to be of high quality, consistent and repeatable under similar conditions. 


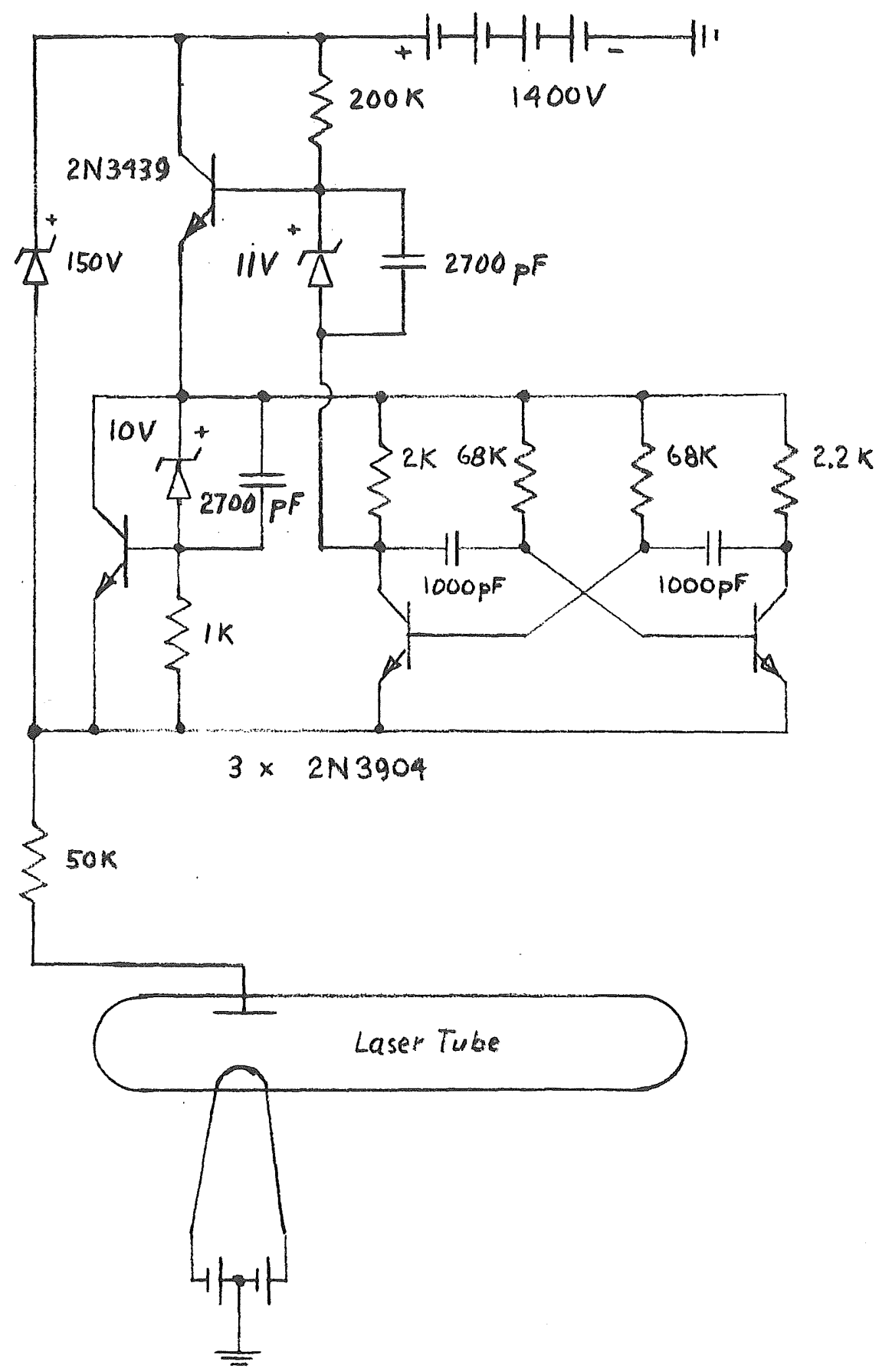

Figure 16. Laser modulator clrcult diagram. 
The experiments are conducted following a standard operating procedure, in order to provide a basis for comparison of results of different days or runs. Simultaneous data at three wavelengths for scintillation probability distribution, variance and covariance of scintillation log amplitude are recorded on the four-track FM tape recorder, to be transcribed into permanent records on the $\mathrm{X}-\mathrm{Y}$ recorder in the laboratory.

The oscillograms in Figure 17 represent three-wavelength linear scintillations. Simultaneous three-wavelength log scintillation is shown in Figure 18. The probability distribution curve shown in Figure 19 has been recorded from tape after a judicious amount of moothing was applied, for one wavelength. These $\mathrm{X}-\mathrm{Y}$ graphs are further handsmoothed and then transferred to Gaussian probability paper. Figure 20 represents the simultaneous three-wavelength probability distribution of log amplitude on such paper. The perfect straightness of the lines confirms the predicted log normality of the distribution of scintillation amplitude at a11 three wavelengths. This is the first time log normality has been proven experimental1y for the far-infrared (10.6 micron wavelength). From these curves it is easy to read directly, besides the distribution, the standard deviation of the distribution (its square is the variance). The deviation from straight lines at the low percentage level is due to the low resolution of the original $X-Y$ plots. (It seems that a sma11 DC shift of the $Y$ axis would straighten out the upper end. A DC shift of the Probability Analyzer output might improve the lower end for two wavelengths.)

Figure 21 shows the simultaneous scintillation spectra of three 


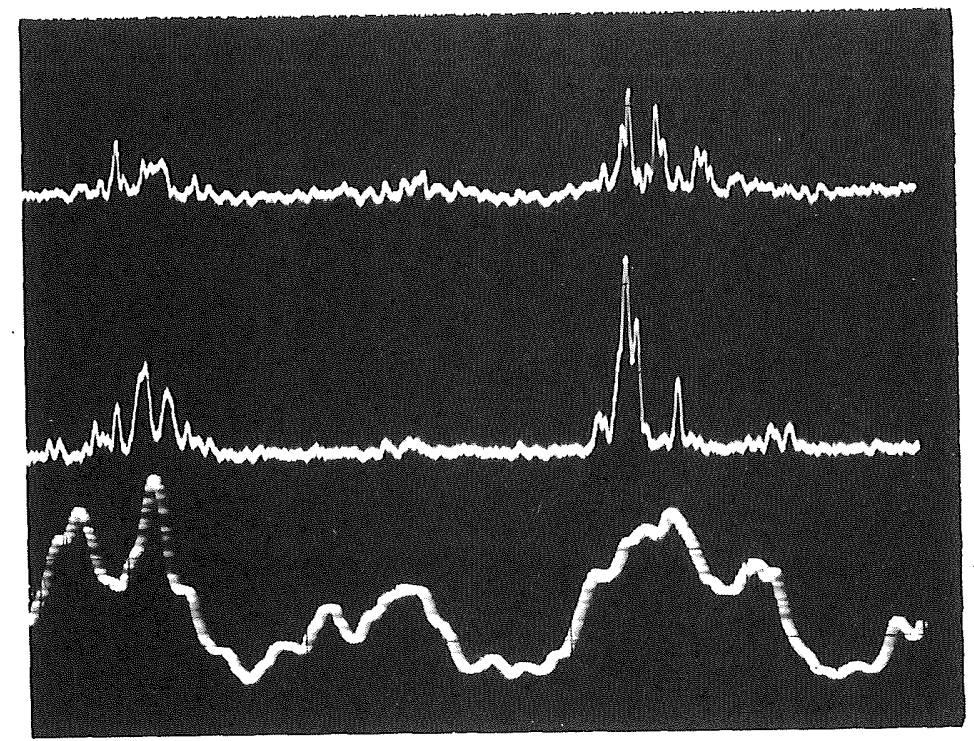

1gure 17. Linear three wavelength scintillations Top: $488 \mathrm{~nm}$, center: $1.15 \mathrm{um}$, bottom: $10.6 \mathrm{um}$.

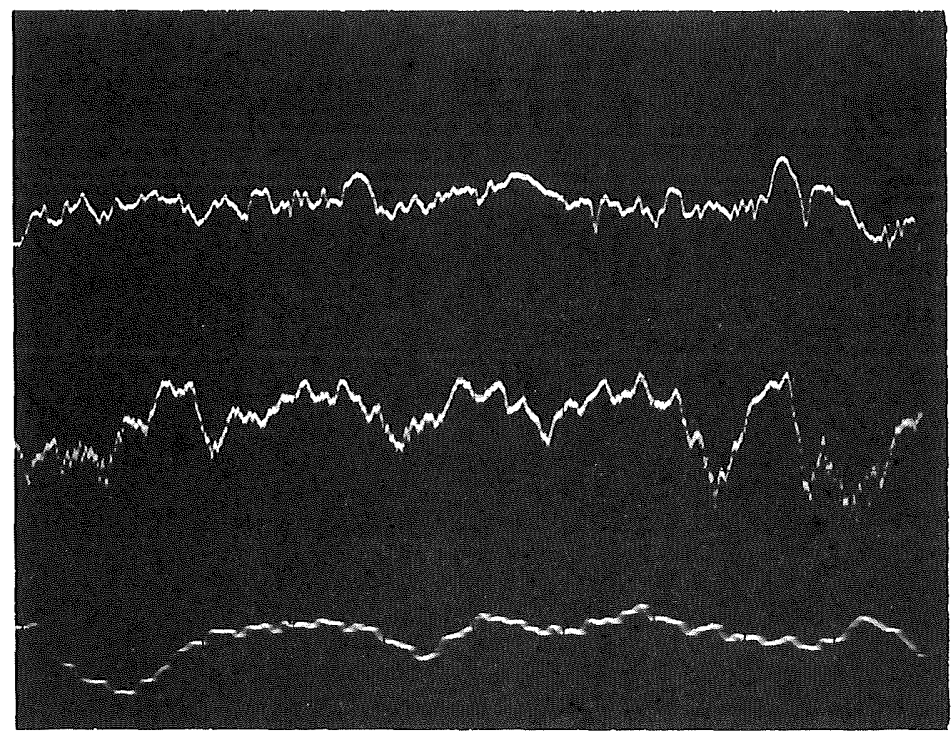

Figure 18. Logarlthmic three wavelength scintillations. TOD: $488 \mathrm{~nm}$, center: $1.15 \mathrm{um}$, bottom: 10.6 um. 
51
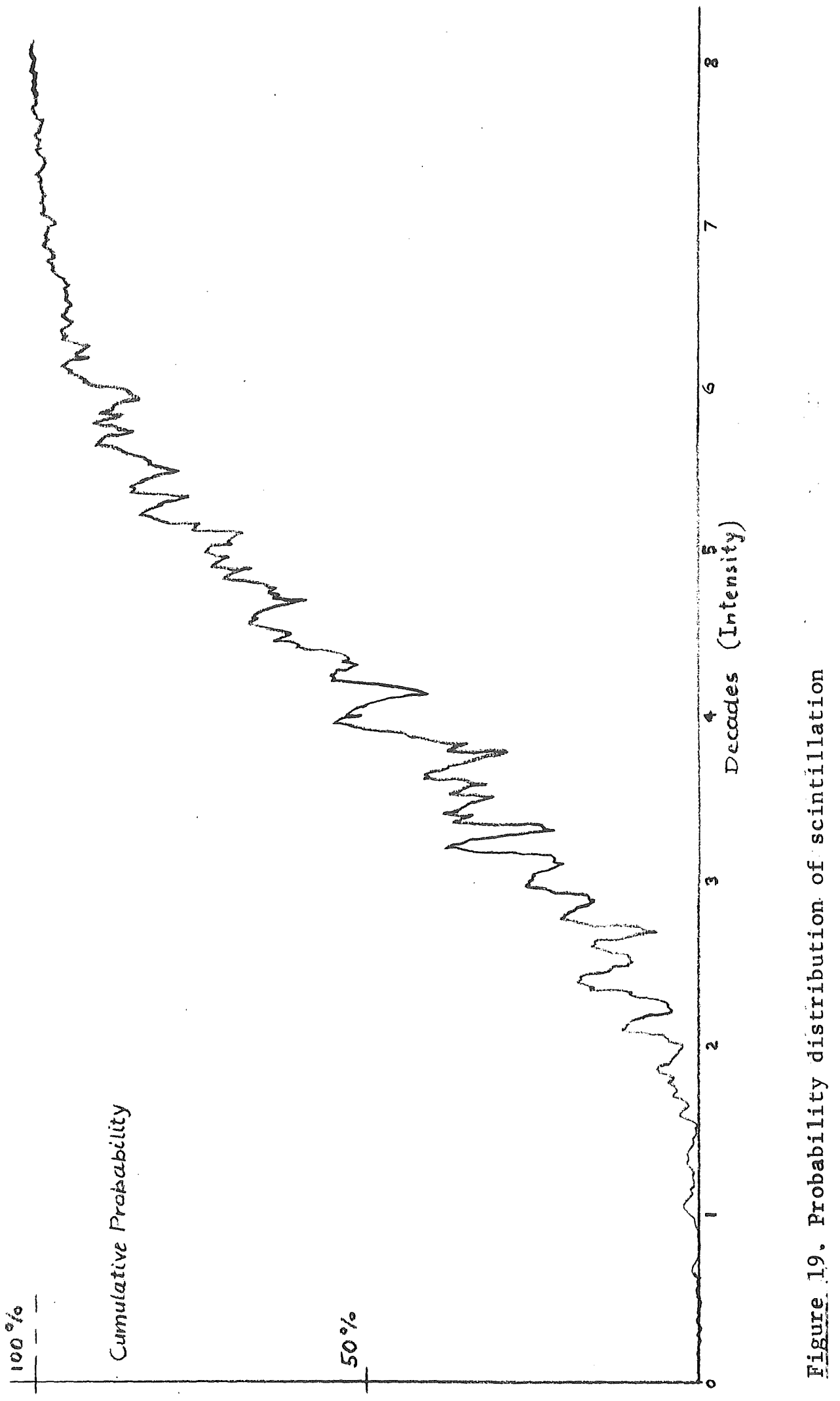

$\therefore$ 


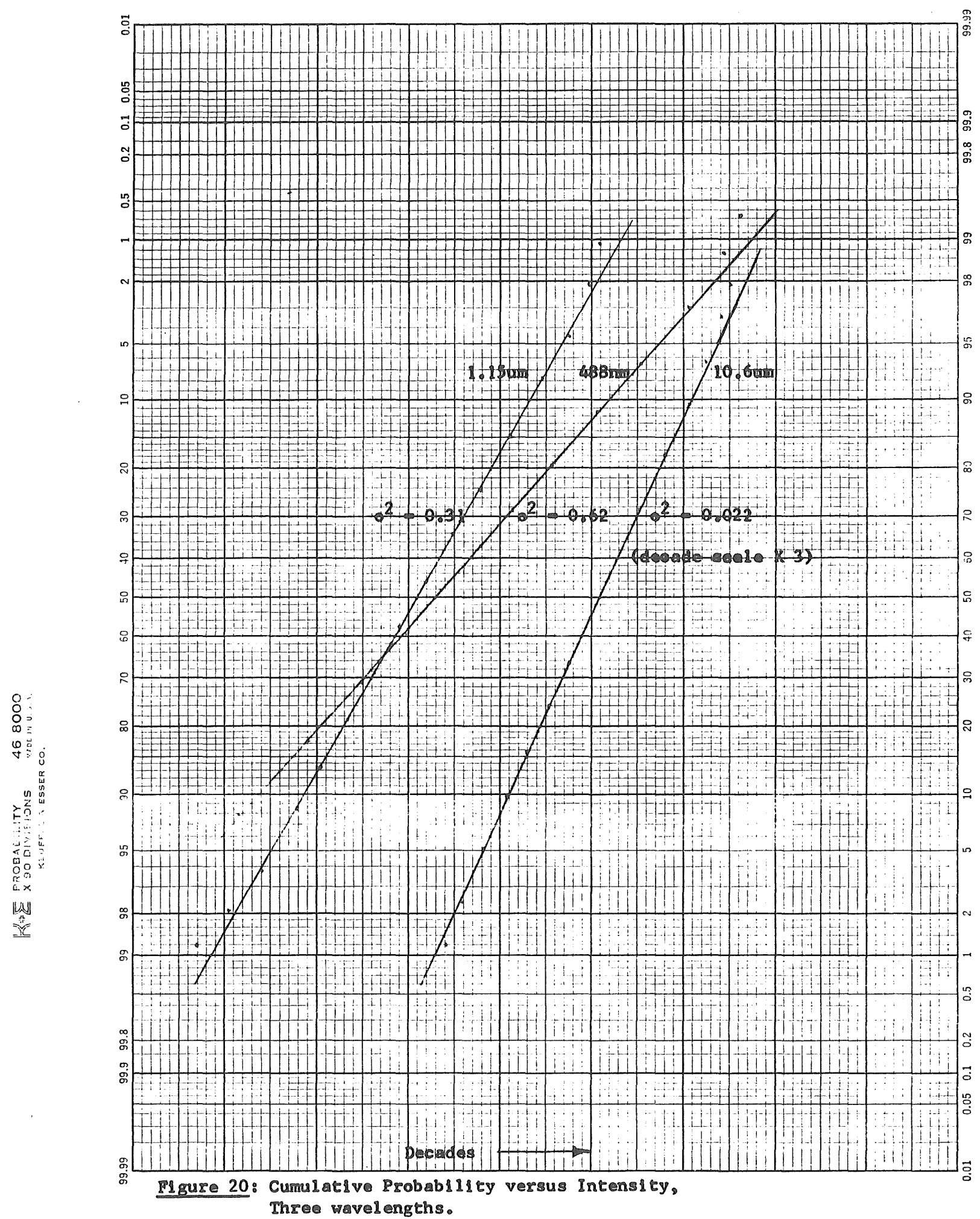




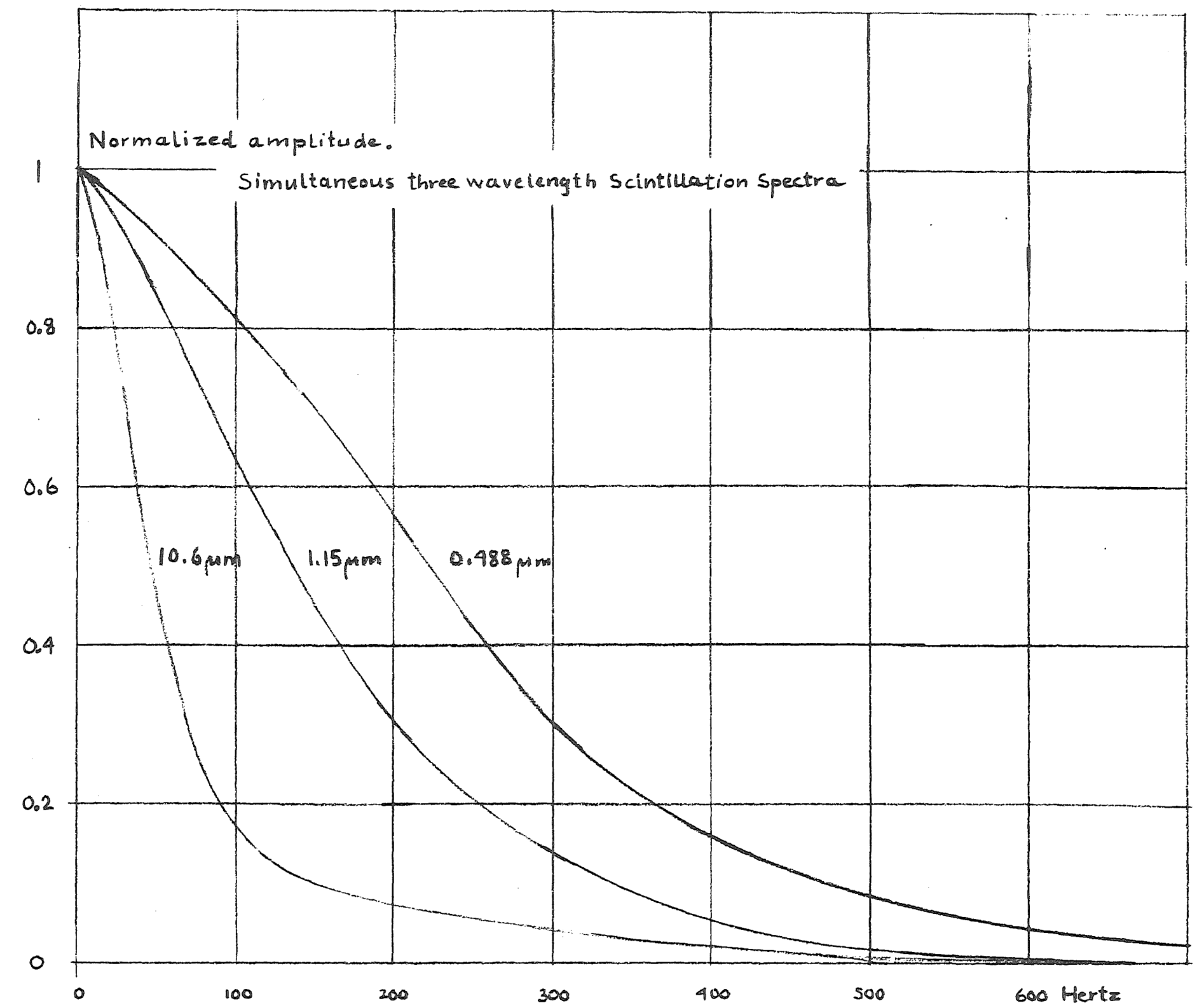

Figure 21. Simultaneous three wavelength scintillation spectra 
wavelengths. The amplitudes have been normalized at frequency $=0$. If the amplitudes were plotted versus modified frequencies $\left(f_{m}\right)$ given by:

$$
f_{m}=f_{0}\left(\lambda / \lambda_{0}\right)^{1 / 2}
$$

where $f_{0}$ is the original frequency, $\lambda_{0}$ a reference wavelength and $\lambda$ the wavelength to which the data applies, the curves for the different wavelengths would cluster closely together.

A similar situation is encountered with the covariances, which are plotted for three wavelengths, versus the sampling aperture separation ( $r$ ) in Figure 22 .

The influence of the light source configuration as characterized by the Fresne1 number $(\mathrm{N})$ is demonstrated in Figure 23. The Fresne1 number indicates how truly a transmitter approximates a point source. Theory predicts a dip near the value of $\mathrm{N}=1$, while experiments in which $\mathrm{N}$ was decreased (by changing the transmitter aperture) from an initial value of 1 , give decreasing instead of increasing values of variance. The two experimental curves shown were obtained at a high and a low refractive index structure constant $\mathrm{C}_{\mathrm{n}}{ }^{2}$. It is hoped that further experimentation will point up the reasons for the apparent discrepancy. 


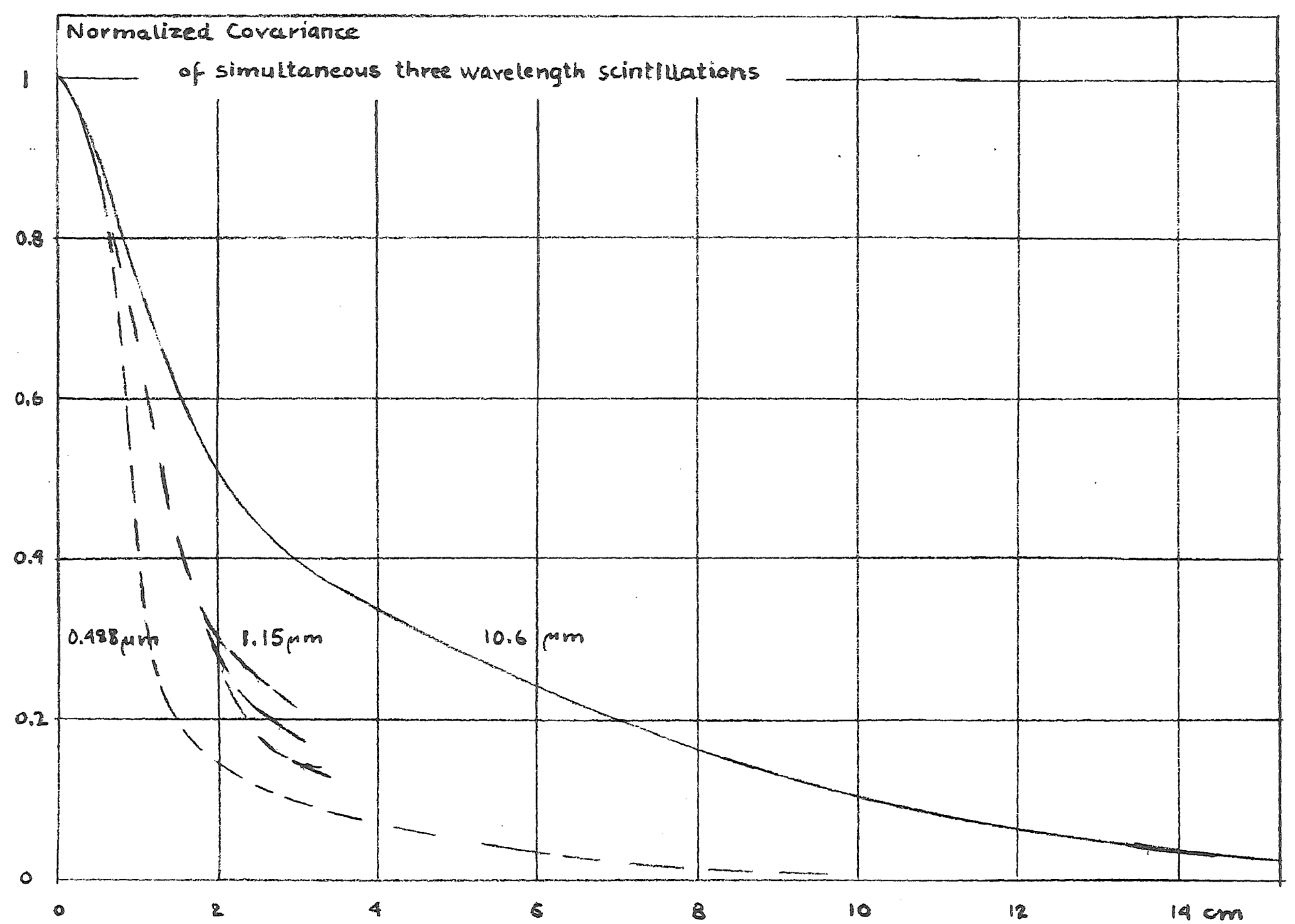

Figure 22. Simultaneous three wavelength covariance of scintillation 


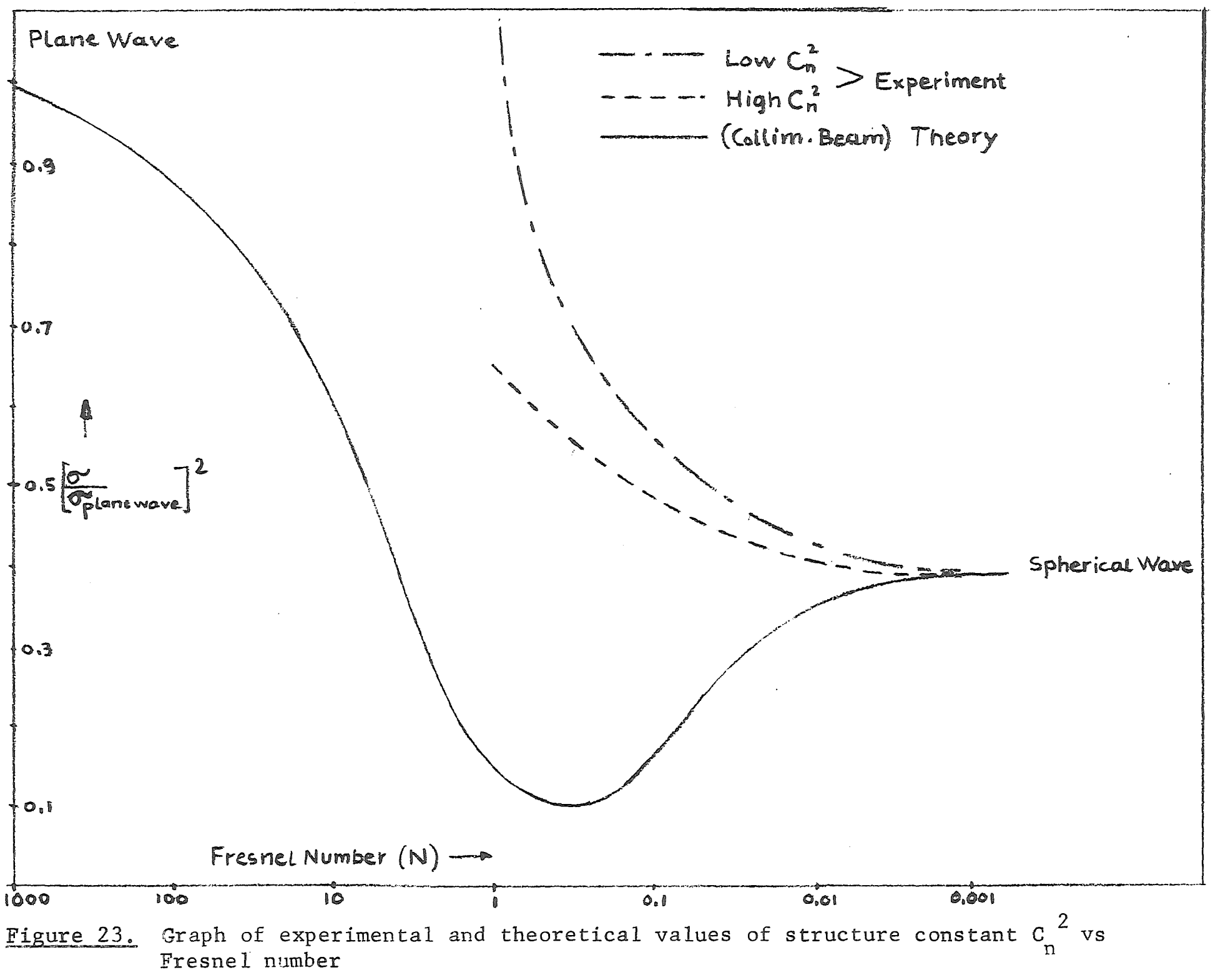




\section{EXPERIMENTAL FACILITY FOR INVESTIGATION OF TURBULENCE PROPAGATION PHENOMENA METEOROLOGICAL SYSTEMS}

In order to correlate properly the optical scintillation results with turbulence theory, it is necessary to determine independently the degree of turbulence, e.g., through direct measurement of the temperature structure function (Chapter II-B). Also, general meteorology parameters must be monitored and recorded. The instruments required to obtain these data were, in part, developed by the author and are described in this Chapter.

\section{A DIFFERENTIAL THERMOMETER}

\section{Genera1 Description}

The differential thermometer shown in Figure 24 uses the close relationship between the local temperature of turbulent air bubbles and the local value of the refractive index as an independent method for the determination of the refractive index structure function and associated statistical data. In Chapter II-B, the connection which links the refractive index structure function to the variance of the instantaneous temperature difference fluctuations between two points separated by a distance $r$ is shown. Here the physical realization is described.

The key element of the differential high-speed thermometer is an extremely thin (and fragile) platinum wire which is able to transduce 


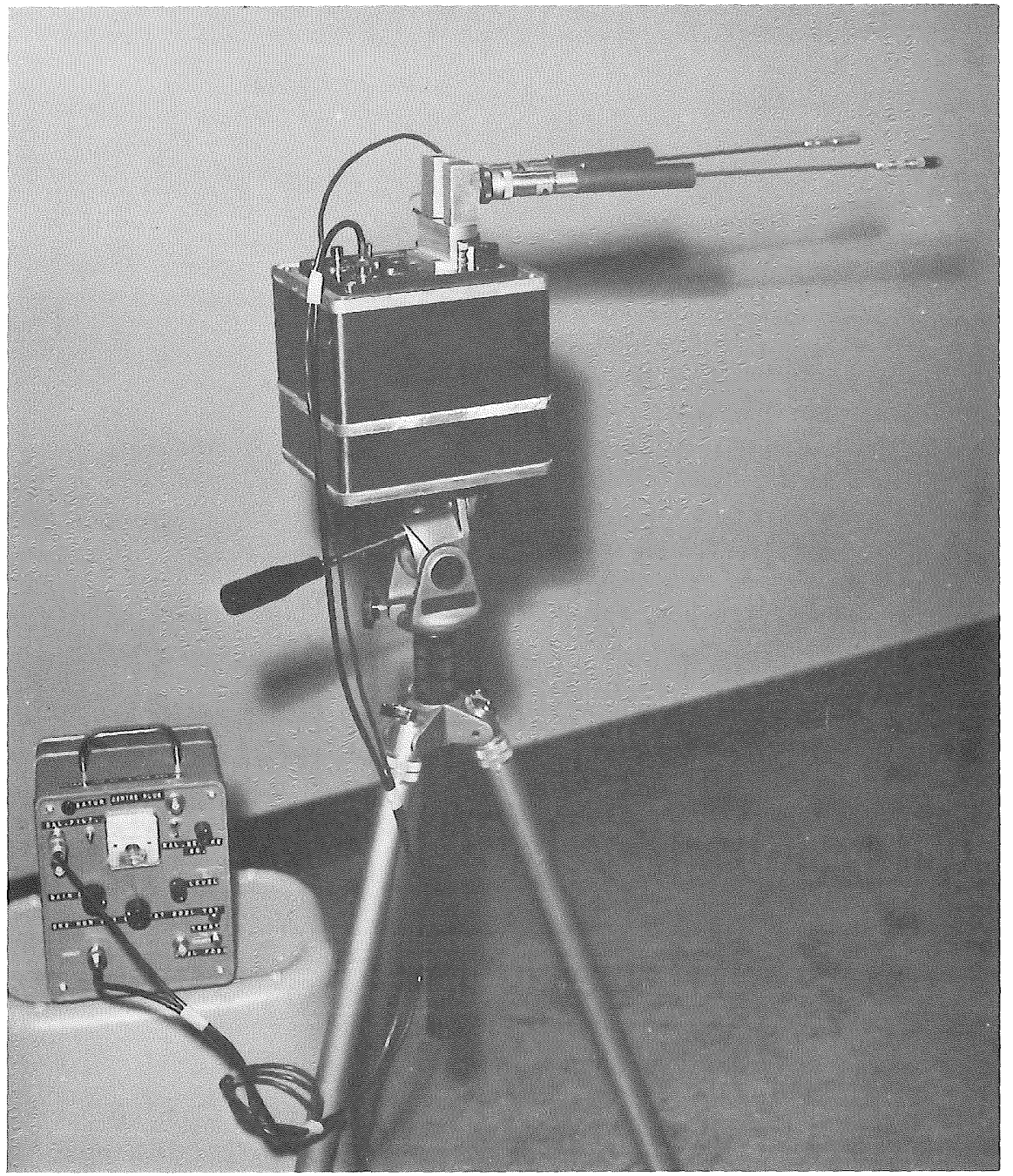

Flgure 24. Refractive Inder structure constant meter 
the temperature fluctuation into an electrical signal (27). The high surface-to-volume ratio of the $3 \mathrm{~mm}$ long and 2 micrometers thick wire facilitates temperature exchange with the environment. In order to disturb minimally the normal air movement the wire is suspended between two short supporting needles, which are mounted at the tip of a 30 centimeter long, thin steel tube, fitted with an electrical plug. A pair of these tubes with probe wires is plugged and fastened into rotatable sockets, permitting the separation of the probe tips to be adjusted like the rods of a rabbit ear TV antenna, from 1 to $50 \mathrm{~cm}$ for direct verification of the previously discussed "two-thirds law" $(6,7)$

The measurement of local air temperature requires that the wire resistance be sensed with little enough power to prevent self-heating, in which case wind velocity would enter the measurement. This effect is so strong that probes of this kind are, in fact, used as anemometers (so-called hot-wire probes). The low sensing power means that the electrical signals collected from the sensing wires are very small and that care must be taken to keep noise contributions small.

Since the temperature fluctuations in which we are interested are in a band between less than $1 \mathrm{~Hz}$ and $1 \mathrm{kHz}$, a carrier modulation technique is used in order to shift the signal out of the region of low frequency noise, which is contributed by the amplifiers.

Figure 25 shows a Wheatstone bridge as a balanced modulator of a constant-voltage audio frequency carrier. The modulated signal is amplified, demodulated and low-pass filtered. The DC component of the filter output is maintained at a prescribed adjustable level (28) by 


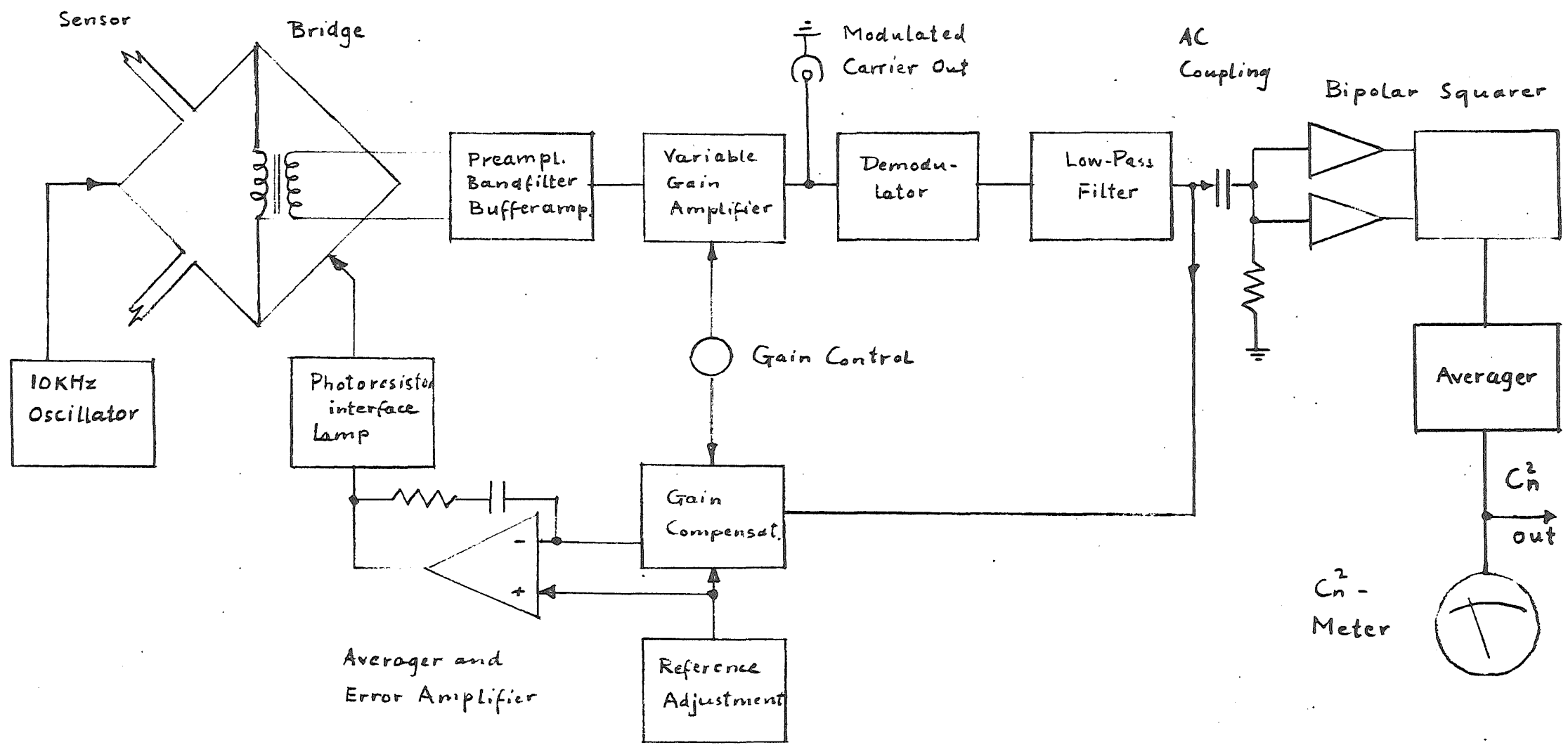

Figure 25. Block diagram of $\mathrm{C}_{\mathrm{n}}^{2}$ instrument. 
feedback control, while the $\mathrm{AC}$ component is squared and the result is averaged and indicated as the variance of the temperature difference fluctuations or the temperature structure function.

The present system incorporates an optimum combination of features which were employed by the other workers cited earlier, in their instruments. One used a single probe, while others incorporated two probes at a fixed separation. Automatic bridge balancing was employed in one case, and a provision for quick calibration was incorporated in a11. Besides the ability of operating without frequent interruptions for readjustment, another advantage derived from the automatic contro1 of the bridge to a state of imbalance (which results in the above mentioned constant average of the filter output DC component) is that demodulation is accomplished with a simple circuit, instead of a synchronous demodulator which requires a phase reference signal.

In the following paragraphs expressions for the optimal noise input resistance to the preamplifier, the signa1-to-noise power ratio for a critical temperature sensitivity, and minimum and maximum probe driving power result from a more detailed discussion of the circuitry.

\section{Bridge Circuit}

The bridge circuit is shown in Figure 26. The two temperature probes and two fixed resistors of 82 ohms (the published resistance of new probes) form the four branches of a Wheatstone bridge. The bridge can be coarsely balanced by a potentiometer constituting two oppositeIy variable shunts across the two sensing branches. The probes, whose resistance was determined at low current as over 100 ohms, are shunted by photo cells. Automatic balancing is done by varying the resistance 


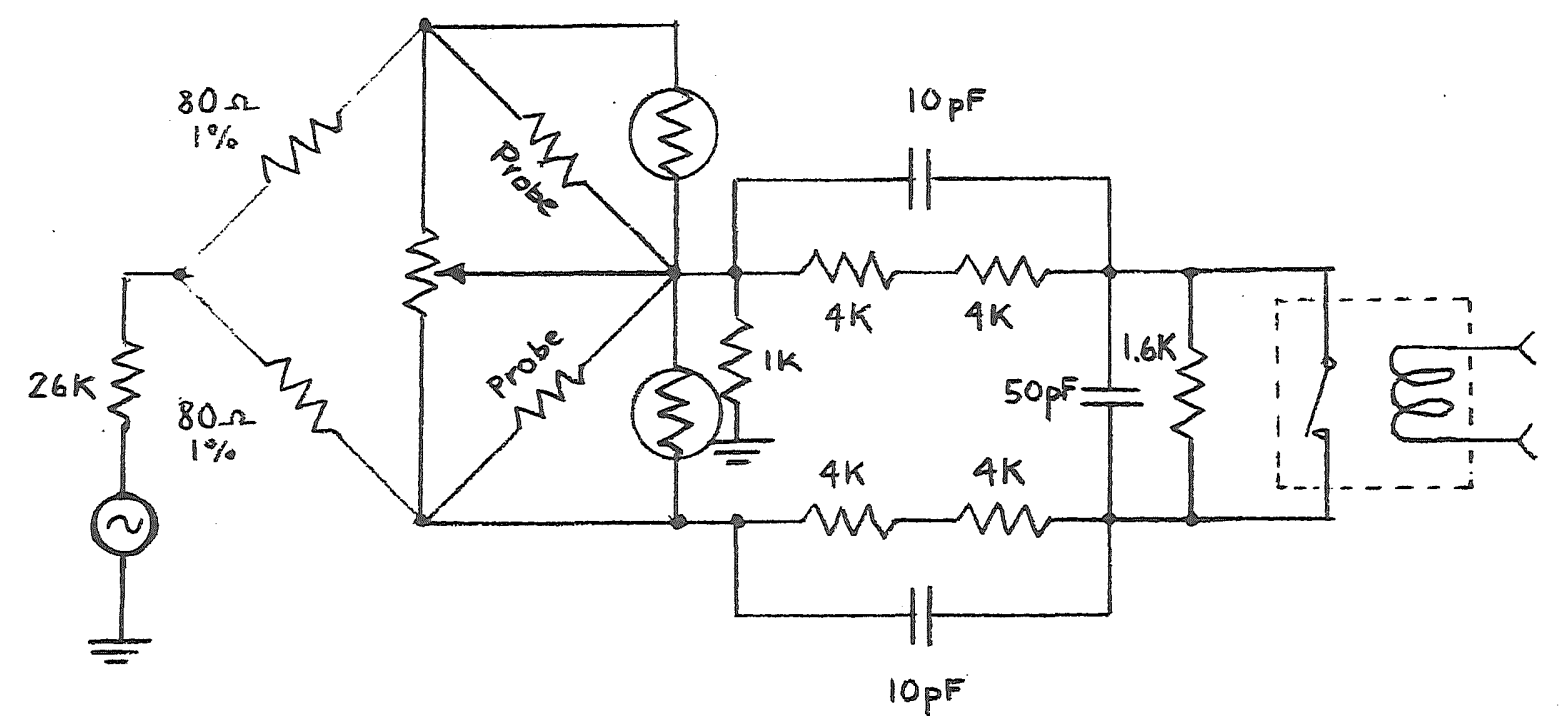

static test

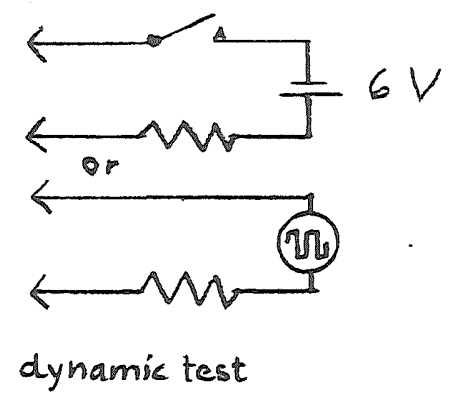

Figure 26. $\mathrm{C}_{\mathrm{n}}^{2}$ detector bridge with sensitivity test circuit 
of the two photocells by the feedback-controlled intensity of the associated incandescent lamps. Reactive imbalance is compensated by the fixed and the variable capacitor from the bridge output terminals to ground. In the following derivations, the capacitors are not taken into account since their reactance is about 200 times that of the branches and the sensitivity of the bridge is only 0.5 percent in error in the results。

A $10 \mathrm{kHz}$, constant-amplitude, precision generator supplies the bridge with a small current $I_{s s}$ determined mainly by a large series resistance $\mathrm{KR}$ :

$$
I_{s s}=\frac{2 V s s}{R(2 K+a+1)}
$$

where $V_{s s}$ is the generator supply voltage (6 volts rms). All resistances are referred to $R$, the constant branch resistance of 82 ohms. With the probe resistances averaging 100 ohms, the factor a is taken to be 1.2. It is convenient to list the equations of a few pertinent parameters for easy reference: For instance, the magnitude of the unloaded bridge output voltage for a small change of sensor resistance $\Delta R$ is:

$$
\Delta V=\frac{I_{s S} \Delta R}{2(1+a)}
$$

This is the open circuit Thevenin equivalent generator voltage. The internal resistance of this generator $\left(R_{g}\right)$ is given by:

$$
R_{g}=\frac{2 a R}{a+1}
$$


Preamplifier and Noise Performance

The preamplifier which simplifies the weak bridge output signals is selected for ultralow noise performance. But this is only attained if its input terminals are connected to a generator of a specified internal impedance equal to the equivalent noise resistance of the amplifier $\left(R_{n}\right)$, which is given as the quotient of the amplifier noise voltage $e_{n}$ and its noise current $i_{n}$ referred to its input. On the other hand, signal power transfer from the bridge is optimized if the load resistance equals the bridge equivalent generator resistance $R_{g}$. For DC stability reasons it is preferable to insert the needed resistance between the secondary of the matching transformer and the amplifier input terminals 。

For a transformer ratio $1: N$ between primary and secondary winding and a series resistance $\left(R_{S}\right)$ in the secondary circuit, the above mentioned requirements of optimum signal power and low noise are satisfied if the following two equations hold:

a)

$$
N^{2} R_{g}+R_{s}=R_{n}
$$

b)

$$
N^{2} R_{g}=R_{s}
$$

from which:

$$
R_{s}=R_{n} / 2
$$

$$
3
$$

Symmetry requires that the resistance $R_{S} / 2$ be inserted in series with each of the transformer secondary terminals. 
With the bridge so connected to the preamplifier the signal power dissipated in the input circuit can be compared to the corresponding equivalent noise power. The signal power $\left(P_{S}\right)$ is given by:

$$
P_{s}=\frac{(\Delta V)^{2}}{2 R_{g}}=\frac{(\Delta V N)^{2}}{R_{n}}
$$

The equivalent Johnson noise power $\left(P_{j}\right)$ is

$$
P_{j}=\frac{V_{j}^{2}}{R_{n}}=4 \mathrm{kTB}
$$

where $k$ is Boltzmann's constant, $T$ is the temperature and $B$ is the bandwidth. The equivalent amplifier noise power $\left(\mathrm{P}_{\mathrm{a}}\right)$ (Table II) is:

$$
P_{a}=i_{n}^{2} R_{n}+\frac{e_{n}^{2}}{R_{n}}=2 i_{n}^{2} R_{n}
$$

where $e_{n}$ and $i_{n}$ also correspond to the bandwidth (B). The deterioration of noise performance by the amplifier can be expressed by the amplifier noise figure (NF):

$$
N F=\frac{P_{s} / P_{j}}{P_{s} /\left(P_{j}+P_{a}\right)}=\frac{P_{j}+P_{a}}{P_{j}}=1+\frac{P_{a}}{P_{j}}
$$

Substituting the above values for $P_{j}$ and $P_{a}$, we obtain:

$$
N E=1+\frac{2 e n^{i} n}{4 k T B}
$$

The signal-to-noise power ratio (without the amplifier noise 
TABLE II

NOISE MEASUREMENTS ON OPERATIONAL AMPLIFIERS

\begin{tabular}{|c|c|c|c|c|}
\hline \multicolumn{2}{|c|}{ Type } & $i_{n}$ & $e_{n}$ & $\mathrm{R}_{\mathrm{n}}$ \\
\hline $\begin{aligned} \text { Burr }- \text { Brown } \\
$\[ (6-1000 \]$\end{aligned}$ & $\begin{array}{l}3019 / 15 \\
\mathrm{~Hz})\end{array}$ & $50 \mathrm{pA}$ & $400 \mathrm{nV}$ & $8.0 \mathrm{k} \Omega$ \\
\hline Burr-Brown & $3119 / 12 \mathrm{C}$ & $100 \mathrm{pA} /(100 \mathrm{~Hz})^{1 / 2}$ & $240 \mathrm{nV} /(100 \mathrm{~Hz})^{1 / 2}$ & $2.4 \mathrm{k} \Omega$ \\
\hline Fairchild & $741-\mathrm{C}$ & $350 \mathrm{pA} /(100 \mathrm{~Hz})^{1 / 2}$ & $50 \mathrm{nV} /(100 \mathrm{~Hz})^{1 / 2}$ & $140 \Omega$ \\
\hline $\begin{array}{l}\text { ( } 1 / f \text { noise } \\
\text { the indicat }\end{array}$ & extends be & $\begin{array}{l}\text { ond } 5 \mathrm{kHz} \text {. At } 5 \mathrm{k} \\
\text { rresponding to } 8 \mathrm{k}\end{array}$ & $\begin{array}{l}\text {, noise levels are } \\
\text { and above.) }\end{array}$ & twice \\
\hline
\end{tabular}


contribution, which is easily added to the end results by use of the above noise figure) is expressed below:

$$
\frac{P_{s}}{P_{j}}=\frac{(\Delta V N)^{2}}{R_{n}} / 4 \mathrm{kTB}
$$

This ratio can be rewritten in terms of oscillator supply current $\left(I_{\text {ss }}\right):$

$$
P_{s}=\frac{I_{s s \Delta R N}{ }^{2}}{2(1+a)} / 4 \mathrm{kTBR}_{n}
$$

To ease further manipulations the following relationships are recalled:

a)

$$
\begin{aligned}
R_{g} & =R_{s s} \text { or, respectively, } \\
\frac{2 a R}{1+a} & =\frac{(1+a) R}{2}
\end{aligned}
$$

b)

$$
R_{n}=2 R_{S}=2 N^{2} R_{g}=2 N^{2} R_{S S}
$$

Multiplying numerator and demodulator in equation (4-11) by $a R$, the value of the probe resistance, yields:

$$
\frac{P_{S}}{P_{j}}=\frac{I_{S S}{ }^{2} a R}{4} \frac{(\Delta R N)^{2}}{(1+a)^{2}} / 4 k^{2} \operatorname{TBR}_{n} a R
$$

where the probe drive power $\left(P_{h}\right)$ is recognized in the first term on the right hand side. Further simplified, equation (4-13) takes the form: 


$$
\frac{P_{s}}{P_{j}}=\frac{P_{h}}{(1+a) 16 k T B}\left(\frac{\Delta R}{a R}\right)^{2}
$$

If the left side of this equation is equal to one, the right side defines a critical temperature fluctuation $(\Delta T \alpha)=(\Delta R / \alpha a R)$ associated with the probe drive power (30) and the temperature coefficient of the probe wire $\alpha$ :

$$
\Delta T_{c r}=4\left[\frac{\mathrm{kTB}(1+a)}{\alpha^{2} P_{h}}\right]^{1 / 2}
$$

For instance, with a probe heating power of 1.3 microwatts, $a=1.2$, $\alpha=0.003$ and a bandwidth of $2500 \mathrm{~Hz}$, the critical temperature fluctuation is $0.005^{\circ}$ Centigrade. A comparis on of signal and noise power in the probe resistance alone would have yielded a somewhat lower figure $(0.0032)$.

\section{Probe Drive Power and Influence of Air Velocity}

Conversely, a minimum probe drive power is defined if the minimum temperature fluctuation to be detected $\left(\Delta \mathrm{T}_{\min }\right)$ is given(28). For

$$
P_{h m i n}=\frac{16 \mathrm{kTB}(1+a)}{\alpha^{2} \Delta T^{2}}
$$

the signal-to-noise power ratio equals unity. As the probe drive power is increased, this ratio also increases. But the probe temperature increases likewise and eventually the probes become sensitive to air velocity fluctuations. Under worst case conditions a pulsating wind can cause the filament temperature to change between the environmental 
(air) temperature $\left(T_{a}\right)$ and $\left(T_{a}+\delta T\right)$, where $\delta T$ is the temperature rise of the probe in still air. The unloaded-bridge output voltage correspondingly changes by an amount $V$ given by analogy with equation $(4-2)$ :

$$
V=\frac{I_{S S} \delta R}{2(1+a)}=\frac{I_{S S} a \alpha R \delta T}{2(1+a)}
$$

The corresponding rms value is $1 /(2)^{3 / 2}$ of the above peak-to-peak value. The relationship between temperature rise $(\delta T)$ and probe drive power has been derived from a graph given by Ochs (15):

$$
\delta T=\frac{P_{h}}{U C}
$$

where $U$ is the volume of the considered temperature probe $\left(9.42 \times 10^{-15}\right)$, $\left(\mathrm{m}^{3}\right)$ and $\mathrm{C}$ is a constant $\left(\mathrm{C}=345 \times 10^{9}\right)$ (Watts $\left./ \mathrm{m}^{3}{ }^{\circ} \mathrm{C}\right)$. In order to compare the desired signal power to the combined power of noise and undesired (wind velocity related) signal, the expressions for each are first brought into a convenient form:

$$
\begin{aligned}
& \text { Signal power: } \frac{(\Delta V N)^{2}}{R_{n}}=\frac{\left(I_{s s} \Delta R N\right)^{2}}{4(1+a)^{2} R_{n}}=\frac{P_{h}}{4(1+a)}\left(\frac{\Delta R}{a R}\right)^{2}=P_{s} \\
& \text { Wind ve1ocity } \frac{(\delta V N)^{2}}{8 R_{n}}
\end{aligned}
$$

The ratio of useful to undesirable power is:

$$
\frac{P_{s}}{P_{j}+P_{w}}=\frac{8(\Delta R)^{2}}{(1+a)(a R)^{2}} \times \frac{1}{\frac{128 k T B}{P_{h}}+\frac{P_{h}^{2} \alpha^{2}}{(1+a) U^{2} c^{2}}}
$$


It is maximum when the terms of the sum in the denominator of the last fraction are equal. If $\mathrm{P}_{h}$ increases beyond the value which realizes this condition, the ratio $(4-20)$ decreases more rapidly than it increased before. Equation (4-20) thus defines a maximum probe drive power $\left(P_{\text {hmass }}\right)$, given by:

$$
P_{h \max }=128 \mathrm{kTB}(1+a) U^{2} c^{2} \alpha^{-2}
$$

The values of the parameters indicated above give a maximum probe drive power of 14.15 microwatts. This value is believed to be conservative, because the resistance fluctuations caused by the air velocity changes are not always at the assumed peak-to-peak value $\delta T$, but are probably less than $1 / 4$ of that. Applying (4-20) to this result yields a critical minimum temperature fluctuation $\left(\Delta \mathrm{T}_{\min }\right)$ of $0.00214^{\circ}$ Centigrade. The preamplifier noise changes this figure to $0.0029^{\circ}$ Centigrade.

The frequency response of the probe wires is determined mainly by the thermal resistance of the environing air, compared to which their own thermal resistance is negligible; and on the thermal capacities of both. A theoretical determination is given in the literature (15), which has been tested in two experimental ways, showing a reasonably smal1 disagreement. Therefore, we used one of these methods to find the frequency response of our probe wires. The chopped beam of a smal1 He-Ne laser was directed onto one of the wires, and the amplitude of the filtered demodulator output was observed. Figures 27 and 28 show the obtained waveforms at different chopping rates. A larger number of 
3.3 Hertz

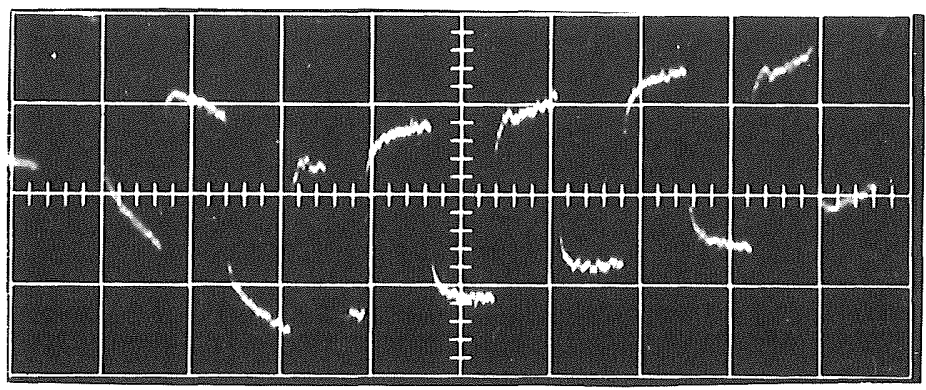

1.5 Hertz

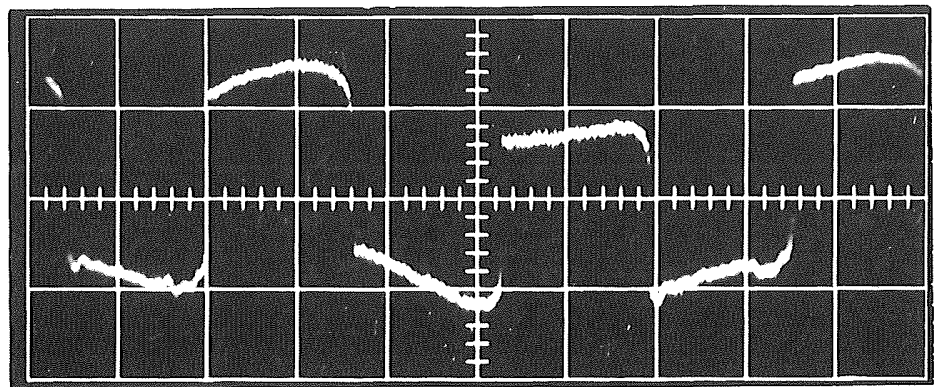

0.6 Hertz

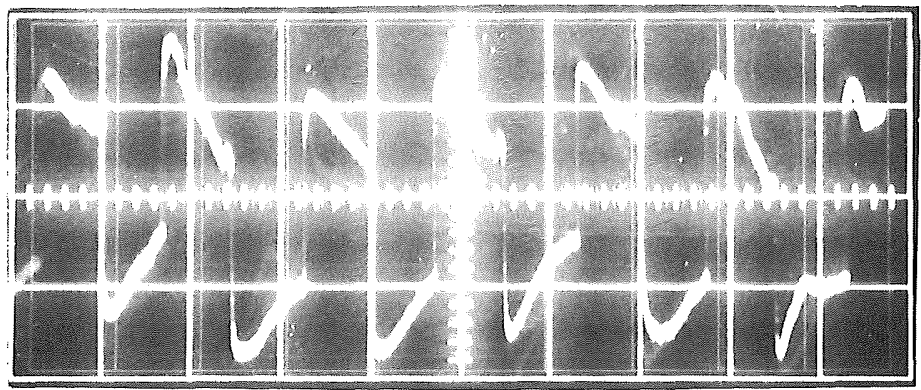

A chopped, unfocussed $5 \mathrm{~mW}$ He-Ne Laser beam illuminates one of the temperature sensing wires. Action of the bridge balance control is evident in the last oscillogram.

Figure 27. Frequency response test waveforms, 1 ow end. 


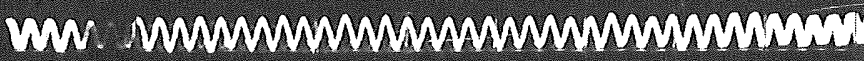

1000 Hertz

\section{munnerve}

333 Hertz

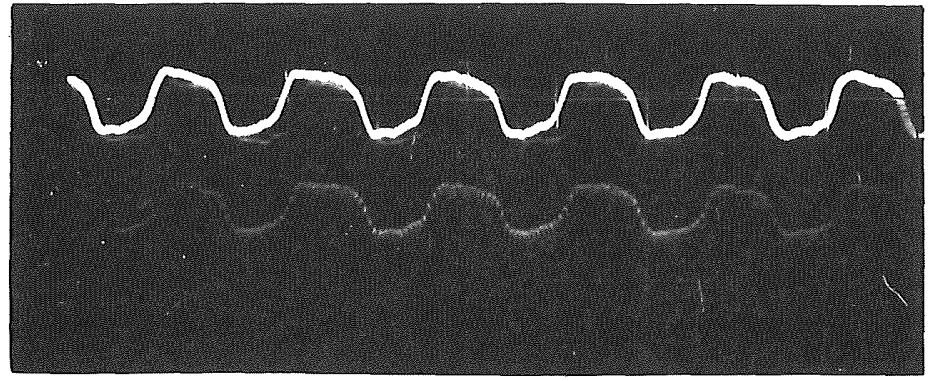

A chopped, unfocussed $5 \mathrm{~mW}$ He-Ne Laser beam 11luminates one of the sensing wires. The faint trace is the modulated carrier. The solid trace is the rectlfied and filtered output.

Figure 28. Frequency response test waveforms, high end. 
readings was taken with a General Radio Tuned Voltmeter in establishing the response curve of Figure 29, which shows a $3 \mathrm{~dB}$ drop of amplitude at $600 \mathrm{~Hz}$. The fast rol1-off is due, in part, to the $2 \mathrm{kHz}$ active filter.

\section{Buffer and Variable Gain Amplifier}

The preamplifier and a buffer amplifier provide enough gain to reach saturation for the strongest input signals, produced by passing a hot iron under the probes. It has been found that even high turbulence does not quite reach this level, and thus the dynamic range is maximized.

The current amplifier preceding the variable gain stage is necessary due to the low input impedance of the latter, which allows the increase in seven steps of the feedback impedance without limitation by circuit and stray capacities. On the contrary, some capacity was purposely added in order to stabilize the phase shift at the highest gain. A11 other feedback resistors have appropriate reactive bypass to equalize the phase shifts for all seven gain positions. The highest resistor is $500 \mathrm{k} \Omega$, which makes the smalles $500 \Omega$. The gain of each switch position has been careful1y trimmed to within 1 percent of its nominal value, since in the later squaring operation the error is doubled.

\section{Demodulator and Filter}

The requirements for the demodulator are much less stringent than was the case in Chapter III-D, because before the signals become too small for accurate observation, the low indication of the meter has forced the operator to switch to the next higher gain position. Since 


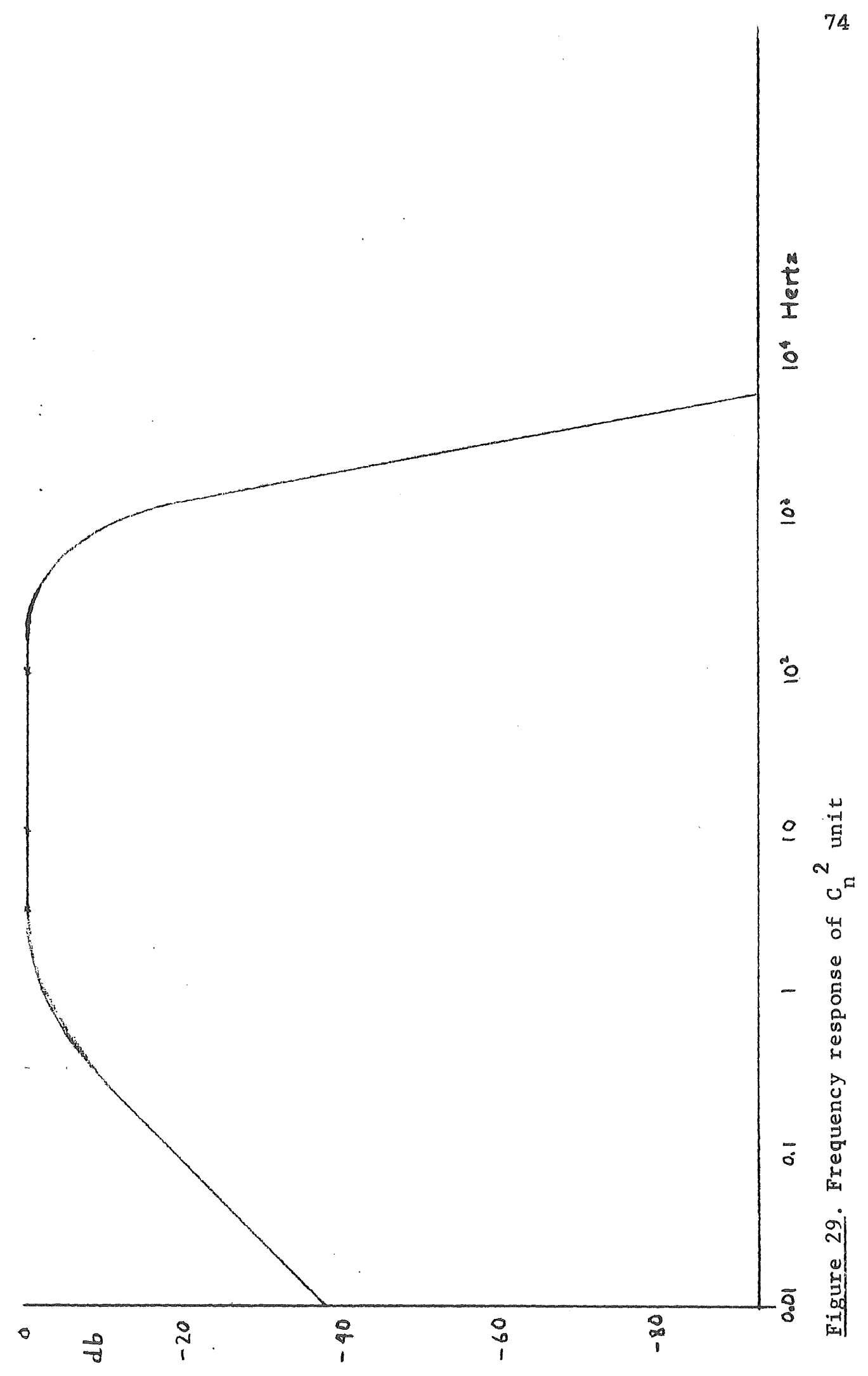


this meter is driven by the square version of the demodulator voltage, it can be seen that the signal range which must be handled by the demodulator is on the order of a decade. A saturation indicator warns the operator when instantaneous signa1 excursions exceed 10 volts, so that the gain may be reduced by one or more steps.

The demodulated signal is smoothed by means of a five-pole, active, low-pass filter. Its output consists of a DC component, representing the average bridge unbalance (28), and a superimposed AC component, which represents the instantaneous fluctuations from this average bridge unbalance. The DC component is held constant by closed-1oop control, while the AC component is capacitor-coupled to the output portion of the system.

\section{Squaring and Averaging}

Negative and positive versions of the AC component are produced by an inverting stage and a voltage follower stage, respectively, with an appropriate voltage gain in order to optimize the accuracy of the squarer. A signal amplitude just reaching the saturation level in previous stages should produce output voltages here which nearly saturate the amplifiers. These output voltages $\left(\mathrm{V}_{\mathrm{sq}}\right)$ are supplied to the unipolar squarer which then produces an output current, given by the equation:

$$
I_{s q}=V_{s q}^{2} / 10^{5} \text { amperes }
$$

Full wave squaring is desirable when dealing with random signals (see Table III)。 
TABLE III

MICROTHERMOMETER SQUARING MODULE PERFORMANCE

\begin{tabular}{|c|c|c|c|}
\hline Input Vo1ts & Expected Output & $\begin{array}{c}\text { Actual Output } \\
\text { Before Bias } \\
\text { Correction }\end{array}$ & $\begin{array}{c}\text { Actual Output } \\
\text { After Bias } \\
\text { Correction }\end{array}$ \\
\hline \pm 1.8 & 0.324 & 0.275 & 0.32 \\
\pm 0.45 & 0.081 & 0.1 & 0.08 \\
& 0.020 & 0.037 & 0.02 \\
\hline & & & \\
Note: & This bias adjustment requires on $1 \mathrm{y}$ occasional check- \\
& ing.
\end{tabular}


The squarer output current is converted into its voltage equivalent so that a current of smaller magnitude can be fed into the averager, making it possible to use a larger value for the feedback resistance, which, together with the integrating capacitor, determines the time constant of the exponentially weighted average (150 seconds). A momentary pushbutton allows the time constant to be changed to 1.8 seconds for quick estimates of the temperature structure function. The size (in $\mu \mathrm{F}$ ) of the capacitors is limited in smallness by the highest value of feedback resistor, which still accurately determines the voltage gain (Appendix $H$ ). The ratio of feedback to input impedance should not exceed $4 \%$ of the open-1oop amplifier gain (K).

\section{Feedback Stabilization}

Since the bridge basically represents a balanced modulator whose output envelope is $|\Delta T|$, rather than $\Delta T$, it is desirable to maintain the bridge in a state of unbalance large enough to assure that in norma1 operation it will never be driven to instantaneous balance (28). There are two advantages to this scheme. First, an ordinary envelope detector can recover the true signal. Second, the carrier average represented by a DC signal out of the demodulator can be used to control the bridge unbalance by merely forcing the low-pass filter output voltage to a fixed value through a closed-loop control. Since there is variable gain in the forward portion of the 1oop, it is cancelled in the feedback path through a coupled switch (Figure 30). This conserves loop stability and response. The following stage then forms an exponentially weightedtime average of the filter output voltage, while the 
To brielge control lamps

From Low Pass Filter

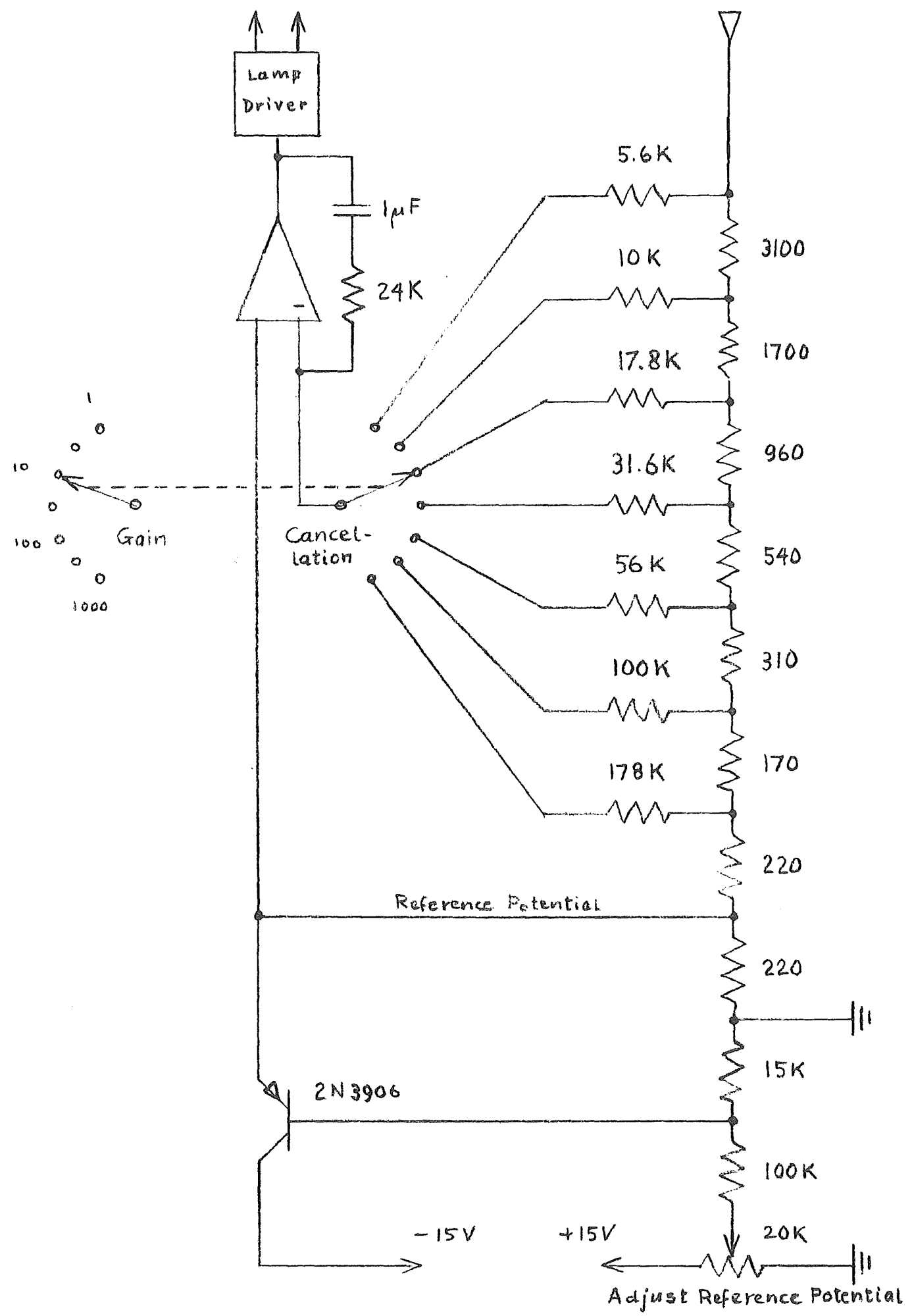

Elgure 30. Gain Cancellation in control loop of $\mathrm{C}_{n}{ }^{2}$ unit. 
non-inverting terminal is supplied with an adjustable reference potential to which the average DC filter output voltage is compared and regulated by means of the feedback mechanism.

The integrator error amplifier controls the current flow in the two incandescent lamps, thus adjusting the resistance of the photosensitive semiconductors across two of the bridge elements. Figure 31 shows graphs relating the control lamp voltage to the photoresistance, the resistance of the shunted bridge branch, and the relative branch resistance in percent of its value at zero lamp voltage. Another curve shows that the bridge sensitivity only begins to be affected $(-2.5 \%)$ when the lamp drive voltage has reached 10 volts.

Figure 32 shows the frequency response of the amplitude and phase of the lamp-photoresistor combination. Note that the magnitude curve is steeper than normal for a single-pole roll-off, because of the nonlinear relationship between the lamp voltage and the photoresistance. The lag between the application of voltage to the lamp and the change in its brightness is due to thermal energy storage in the filament and can be expressed as an RC combination. The amplitude of resistance change varies as a function of frequency, as follows:

$$
R(s)=\frac{R(0)}{1+s^{T}}
$$

where

$$
1 / \mathrm{s}=\tau=0.02 \text { seconds (or } \mathrm{s}=50 \mathrm{radians} / \mathrm{sec} \text { ) }
$$

In order to produce a stable, closed-loop system, the open-loop transfer function must have single-pole (or integrator) behavior, at least up to the frequency where the open-1oop gain is unity. The 

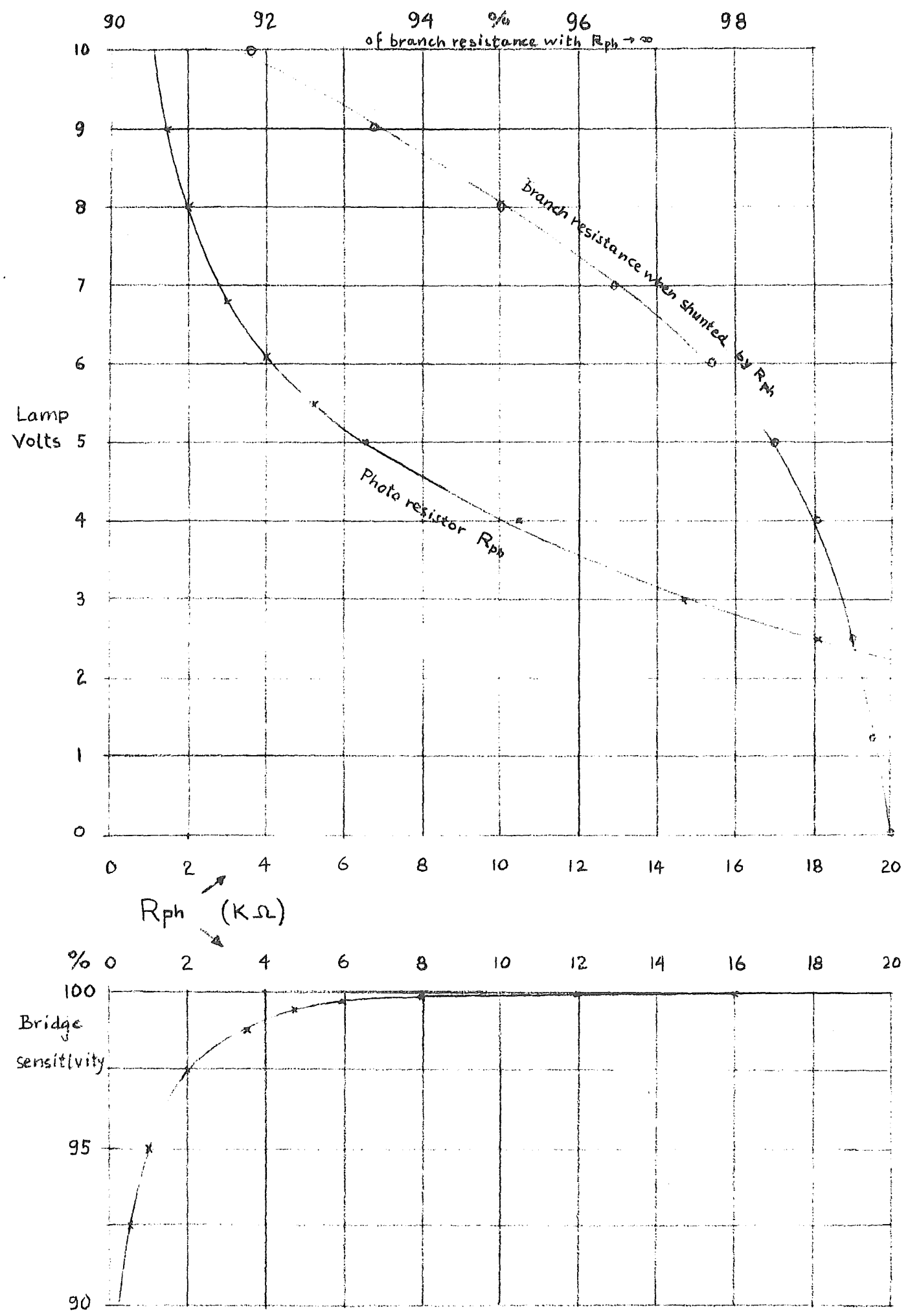

Figure 31. Photoresistance and branch resistance vs lamp voltage and bridge sensitivity vs photoresistance. 

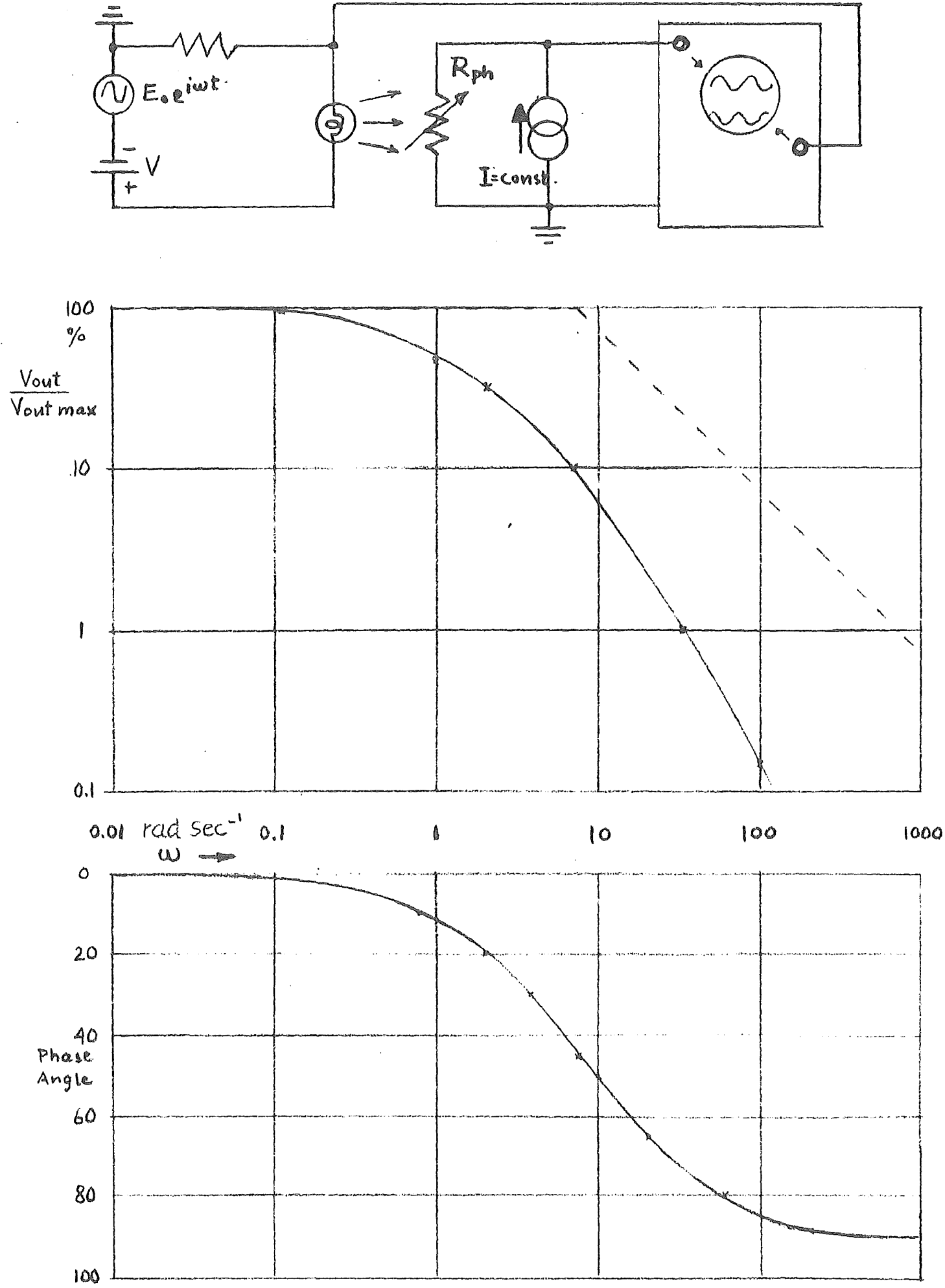

Figure 32. Graphs of magnt tude and phase angle vs frequency (or AC component drIving 1 amp, DC component 1 mplled.) of photoresistor voltage. 
rol1-off must begin at, or below, the lowest frequency at which the temperature fluctuations are to be represented in the measurement. The earlier mentioned integrator and error amplifier (shown in Figure 33) is modified so that the $-6 \mathrm{~dB} /$ octave roll-off of its gain changes into a uniform gain above $50 \mathrm{rad} / \mathrm{sec}$, at which point the lamps begin to roll off. The other blocks in the loop have constant gain at the frequencies of interest to the feedback loop. Thus, loop stability is obtained. The oscillograms in Figure 34 show marginally stable and stable contro1 loop responses to a step input.

\section{Maintenance and Operation}

The instrument is equipped with rechargeable batteries ( $\mathrm{Ni}: \mathrm{Cd}$ ). Charging can be effected overnight or during operation, from a voltageand current-1imited power supply (29 volts and $100 \mathrm{~mA}$ ). Diodes protect the batteries from accidental short and wrong polarity connection of the charging power supply. Newly charged batteries can sustain continuous operation for at least five hours. It is recommended that a trickle charge be used after each prolonged operation, to keep the batteries in good shape.

The following steps are taken in order to properly operate the instrument $\left(\mathrm{C}_{\mathrm{n}}{ }^{2}\right.$ unit): (1) set up the probes in a plane normal to the wind direction; (2) set the tripod at the desired height; (3) disable the automatic bridge balance control; (4) remove the sma11 protective caps from the probes; (5) slide the large diameter tubes over the probes, and plug them, to keep the air around the probes steady; (6) make resistive and capacitive balance adjustments; and (7) engage the feedback. Now the instrument is ready for operation. 


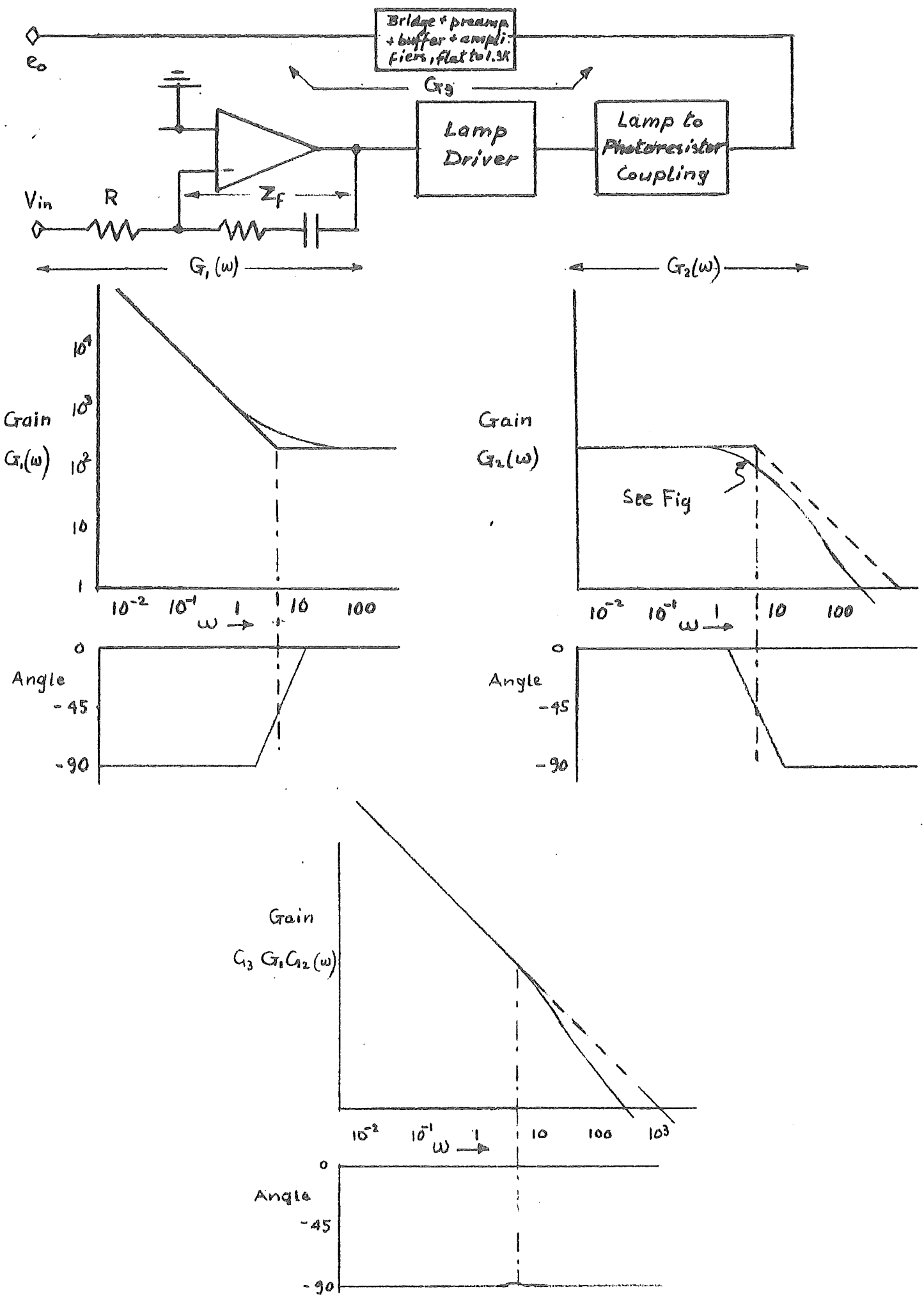

Figure 33. Composition of open loop gain, bridge balance contro1 


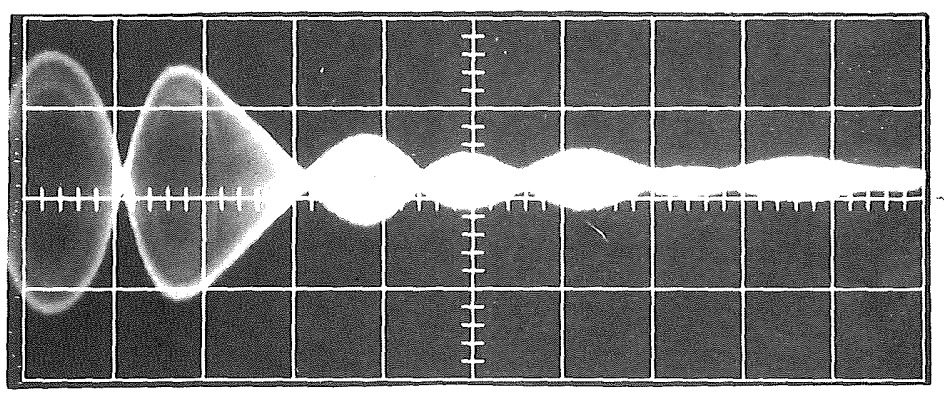

Underdamped

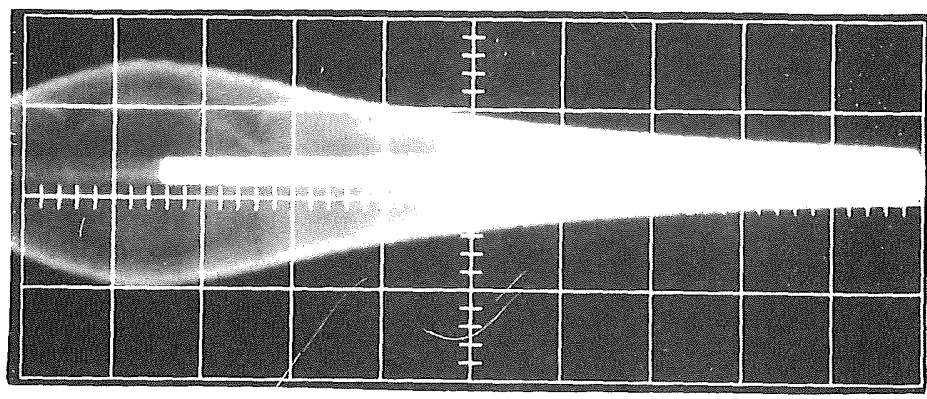

stable

Flgure 34. Stab11ity of bridge balaneing control 1oop. 
When exposing only one probe to the air, the variance of absolute temperature fluctuations is indicated by the meter. With both probes exposed, the meter indicates the temperature structure function, interpretable directly as the refractive structure constant $C_{n}{ }^{2}$ (Chapter II-B, 1-11). The output of the variable gain stage is connected to a jack, from which it can be linked to the scintillation computer. In this way, probability distributions and spectra can be obtained. The probability distributions, smoothed and plotted on probability paper, provide a second value for the temperature structure function and, hence, the refractive index structure constant $\left(C_{n}^{2}\right)$.

\section{Ca1ibration}

Two methods were used to correlate the temperature difference fluctuations between the two probes to the meter reading. One consisted in tracking the gain throughout the instrument, using some fixed hypothetical temperature difference between probes, simulated by a square-wave modulation of the bridge output. This serves mainly to check the proper behavior of each stage.

The modulation was achieved by connecting and disconnecting a precisely tailored shunt across one of the bridge branches, by means of a reed relay operated at a low frequency. In order to get equal timing for open or closed conditions, a square wave was used to drive the relay. The string of shunting resistors is bypassed by capacitors which swamp the influence of relay or stray capacity. The RC constant is the same in each cel1. Periodical1y, $1.6 \mathrm{k} \Omega$ of the total $17.6 \mathrm{k} \Omega$ is shorted, leaving $16 \mathrm{k} \Omega$ across the probe resistance, found to average 
100 ohms rather than 80 , the published value. The resistance change in the branch $(\Delta R)$ is given in terms of the probe resistance $R$, the (short) string resistance $r$ and the (long) string resistance $r(1+b)$ by:

$$
\Delta R=\frac{\operatorname{Rr}(1+b)}{R+r(1+b)}-\frac{R r}{R+r}
$$

With $R=100 \Omega, r / R=160, b=0.1$, the resulting resistance change is $0.561 \times 10^{-3} \mathrm{R}$, which corresponds to a temperature difference between probes of $0.187^{\circ}$ Centigrade. This is about 50 times the minimum detectable value. The output meter reading is $490 \mu \mathrm{A}$ with the gain switched to $100 \mathrm{X}$.

A static test, using the same arrangement and relay to produce a minute resistance change when the relay is energized by pressing a momentary pushbutton, gives a change of demodulator output voltage, which is displayed on the meter when the function switch is in the appropriate position. With the gain set at $100 \mathrm{X}$ and the reading adjusted to mid-scale $(250 \mu \mathrm{A})$, the deflection is $180 \mu \mathrm{A}$. 


\section{B. GENERAL METEOROLOGICAI INSTRUMENTATION}

\section{Relative Humidity Indicator}

The relative humidity indicator makes use of a unique, highly reliable sensing element (30) which is not yet commercially available.

To avoid polarization of the element, it must be driven by alternating current. The frequency is low to keep the capacitive current component sma11. The relative component is most responsive to the relative humidity of the surrounding air.

A simple circuit (Figure 35) uses the sensing element as an input resistor of a feedback amplifier. The generator voltage is a stabilized sinusoid of 80 Hertz. The output voltage which is proportional to the input current is recorded.

To calibrate the indicator, four artificial environments at 20, 40,60 , and 80 percent relative humidity (at $25^{\circ} \mathrm{C}$ ) were produced by putting $100 \mathrm{cc}$ of the appropriate mixture of glycerin and water in tightly sealed plastic quart bottles. The relationship between the specific gravity and the relative humidity produced in the air above it is given in the graph of Figure 36. The sensor to be calibrated is plugged into the socket in one of the four lids, and the lid is then put back on the bottle. The establishment of equilibrium relative humidity is observed on the recorder. Within 10 to 20 minutes the system is stabilized at the new value, which is recorded on the chart, next to the trace. Then the sensor is changed to another bottle and another recording is made. This process is repeated several times with a random variety of room 


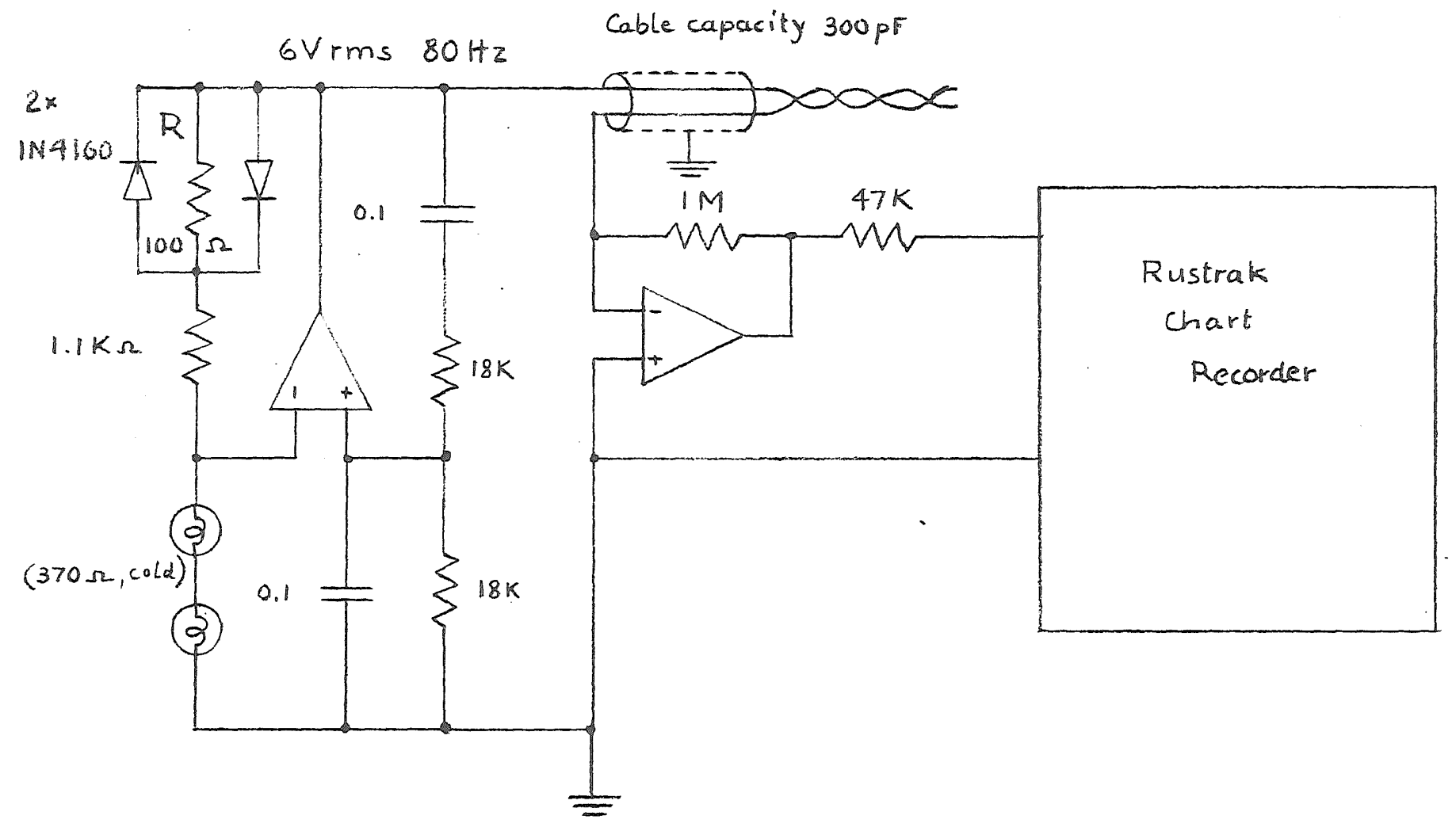

Figure 35. Relative humidity indicator and recorder 


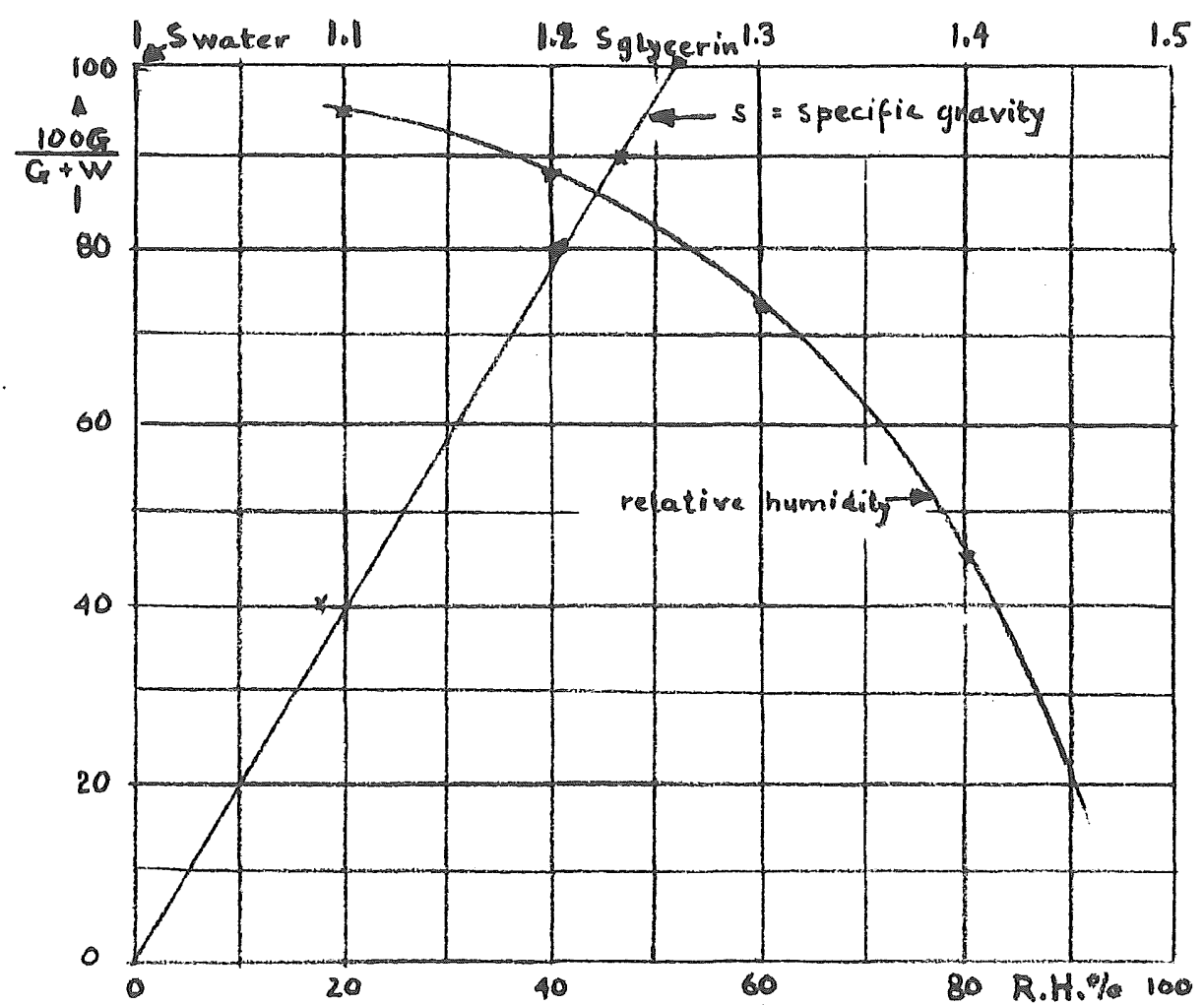

Figure 36. Glycerin content of Glycerin Water mixture vs Relative Humidity and Specific Gravity.

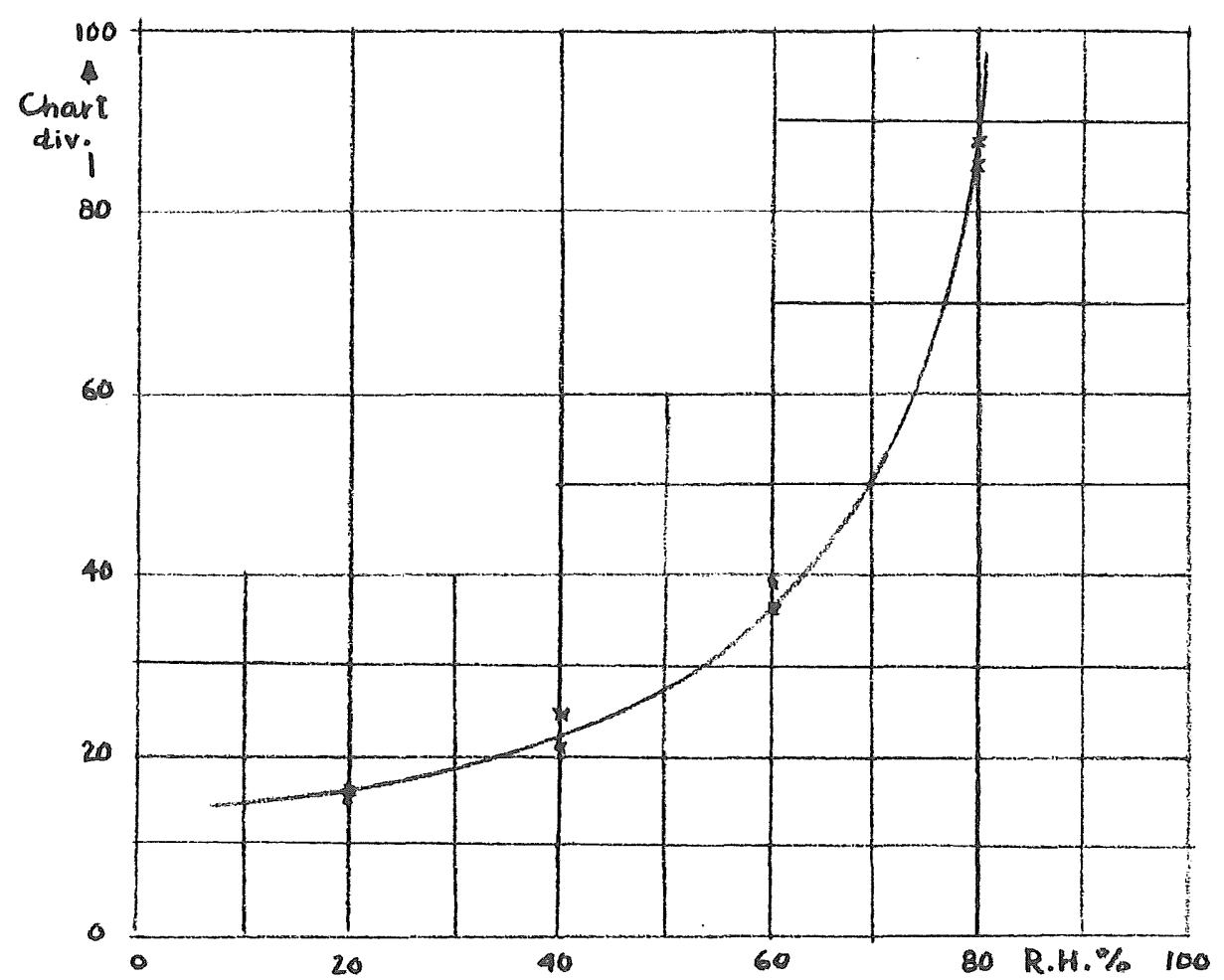

Figure 37. Relative Humidity vs Recorder Chart Divísions. 
temperature. The recordings were repeatable, and quite independent of temperature. The calibration curve shown in Figure 37 reveals a pronounced non-1inearity which makes low readings hard to interpret. The logarithmic circuit shown in Figure 38 remedies this situation.

For use outside a building, it is important that water (raindrops) be kept away from the element.

The we11-known, simple Wien-bridge oscillator is provided with double negative amplitude feedback (Figure 39). First, a higher output voltage produces more calories in the lamp. Their resistance grows ramp-like, producing gradually more negative feedback unti1 a new equilibrium condition is reached. To speed up this process, a small resistor $R_{1}$ in series with the feedback resistor $R_{2}$ is shunted by two diodes which barely begin to conduct current in normal operation, to keep nonlinear distortions low. When the output voltage decreases, they cease to conduct altogether, immediately reducing the feedback, while with increasing output voltage, the diodes instantly conduct more current and the feedback voltage increases. In this way, very low frequency oscillations, frequently observed on the regular circuit, are suppressed. The "integral" control by means of the lamps combined with the proportional action of the diode gives excellent stability of amplitude over a wide range of supply voltages, with very low distortions.

\section{Anemometer}

The metering circuit of an inexpensive commercial rotating-magnet anemometer and direction indicator was modified to permit the continuous recording of wind speed and direction. The instrument delivers 


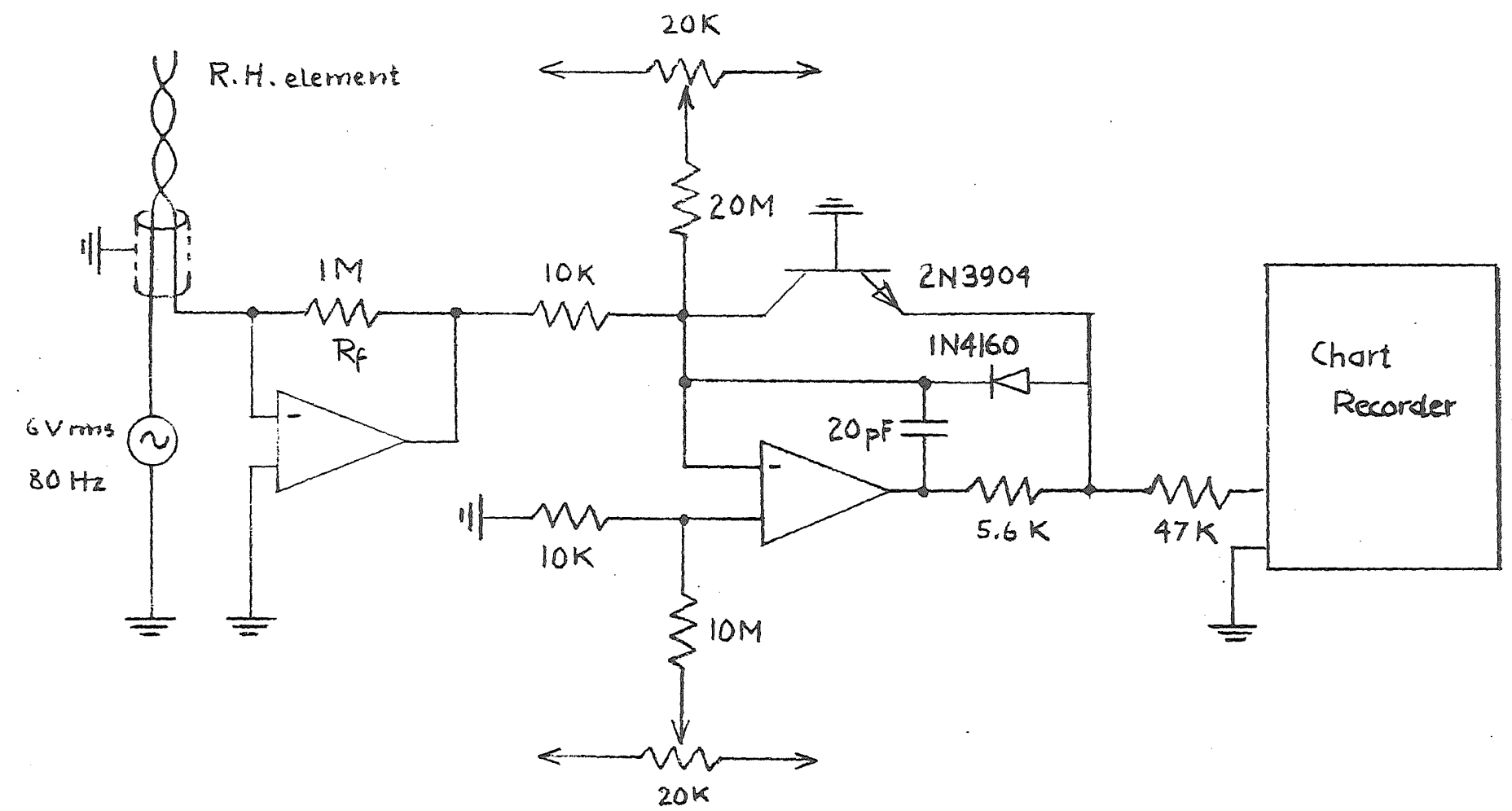

Figure 38. Relative humidity indicator and recorder of improved linearity 


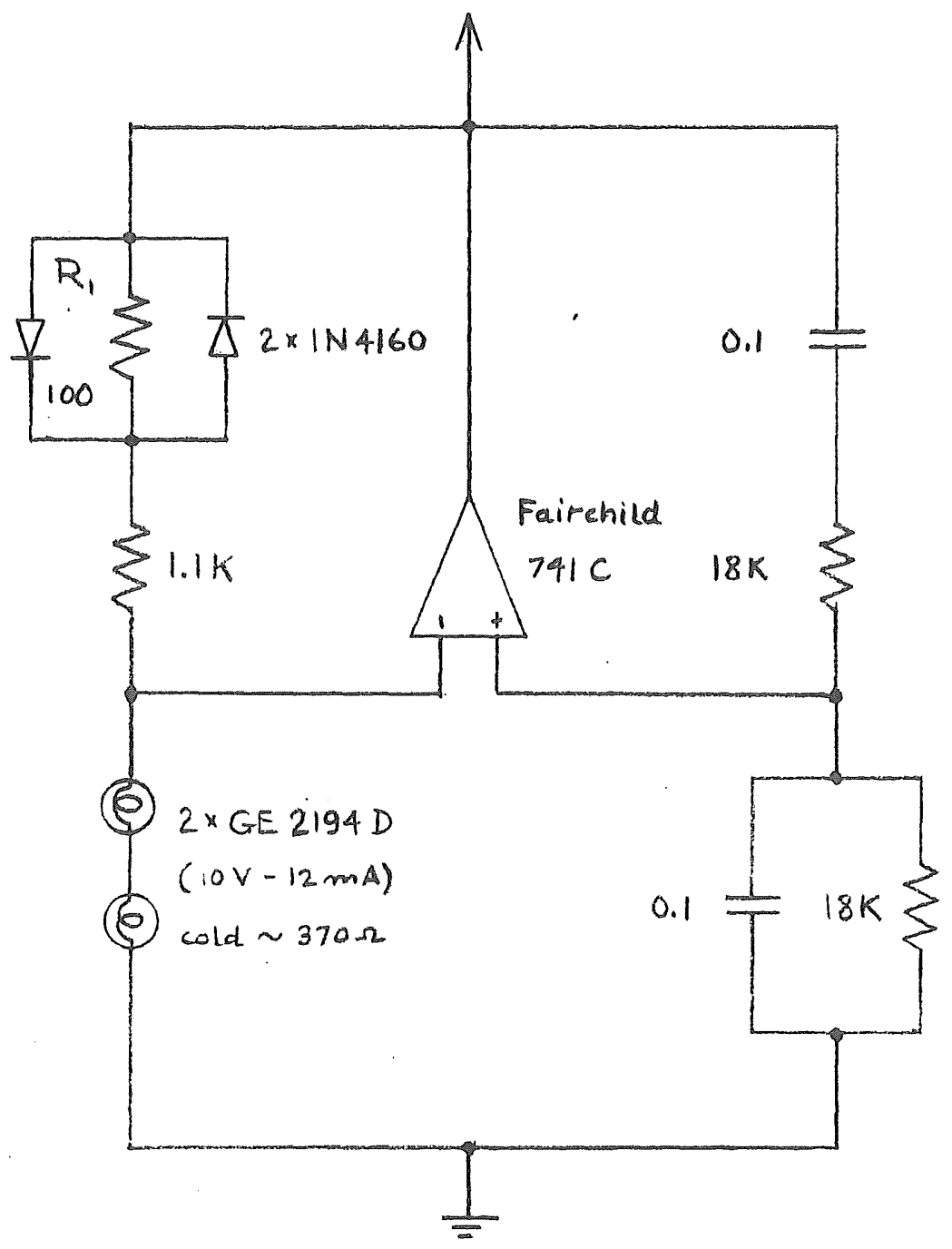

Flgure 39. Stable, low distorslon sinewave generator 
peak electromotive force (and frequency) directly proportional to the wind velocity. The emf sine wave is rectified in a circuit similar to the one described in Chapter III $-D$, with good precision, using a high time constant (Figure 40). The wind direction is indicated potentiometrically.

A wiper fasteneed to the vane makes contact with a resistance track and assumes the potential corresponding to the angle at which it touches. This system showed signs of weakness. Therefore, a combined wind velocity and direction indicator of higher accuracy and with faster response, using strain gauges, is proposed. Such a system has been operated in crude form by a research team at Tektronix, Inc. (31). Pressure and Temperature Indicators

A commercial recording barometer and remote-sensing thermometer complete the meteorological instrumentation.

\section{EXPERIMENTAL RESULTS (32)}

Typical turbulence signals (modulated carrier) are shown in Figure 41. Their dynamic range is very wide. The occasionally nonstationary character of the temperature structure function is demonstrated in the probability distribution shown in Figure 42 . While the data were being recorded, the distribution changed in the middle of the run. The refractive index structure constant was determined from the slopes of such curves for six runs. The results were found to agree within a reasonably steady factor (not exactly constant), with the meter readings for the same variable (Table IV), when the distributions were Gaussian. In other cases, it is difficult to interpret 


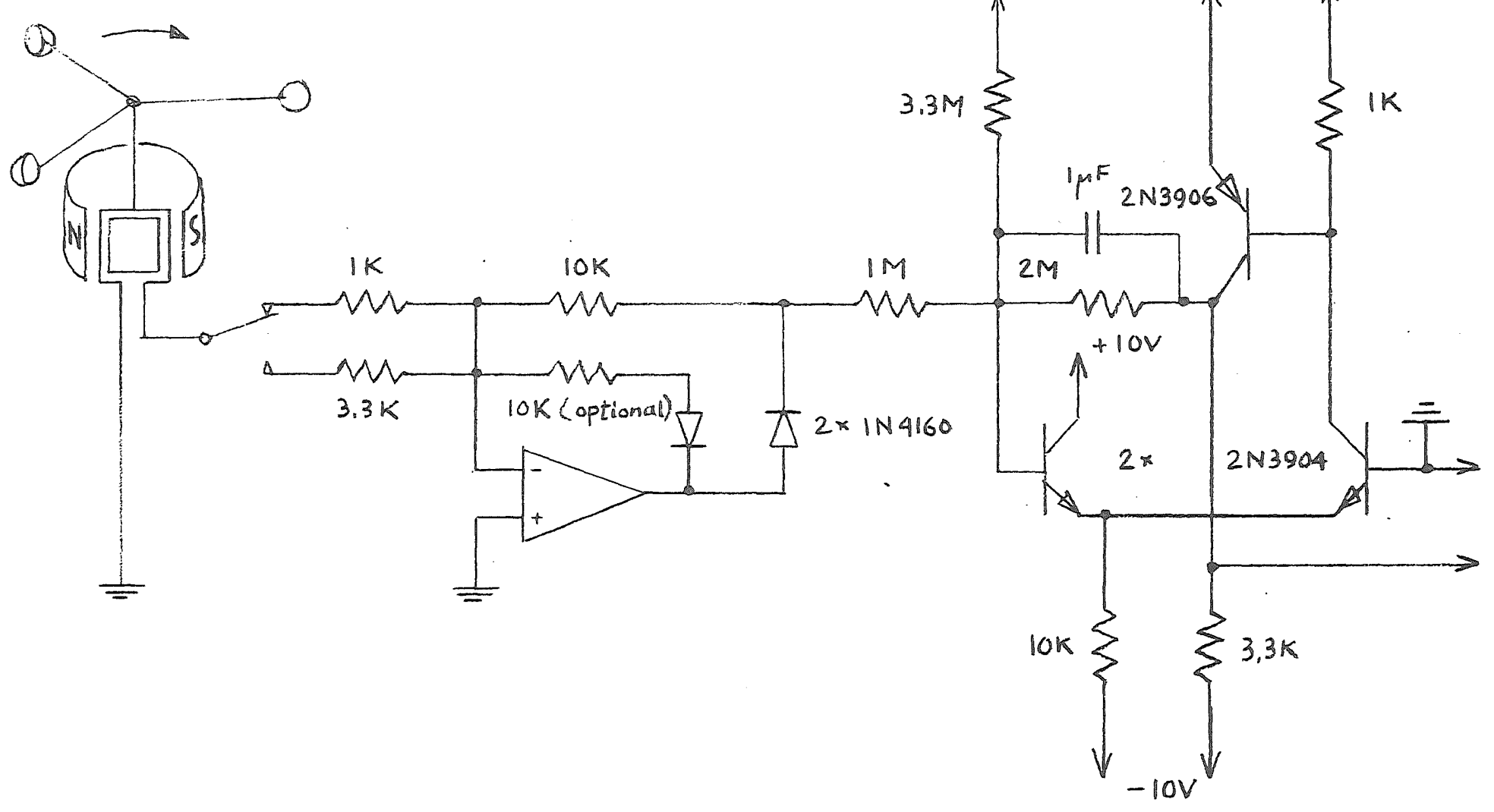

Ifure 40. Anemometer demodulator circuit diagram 

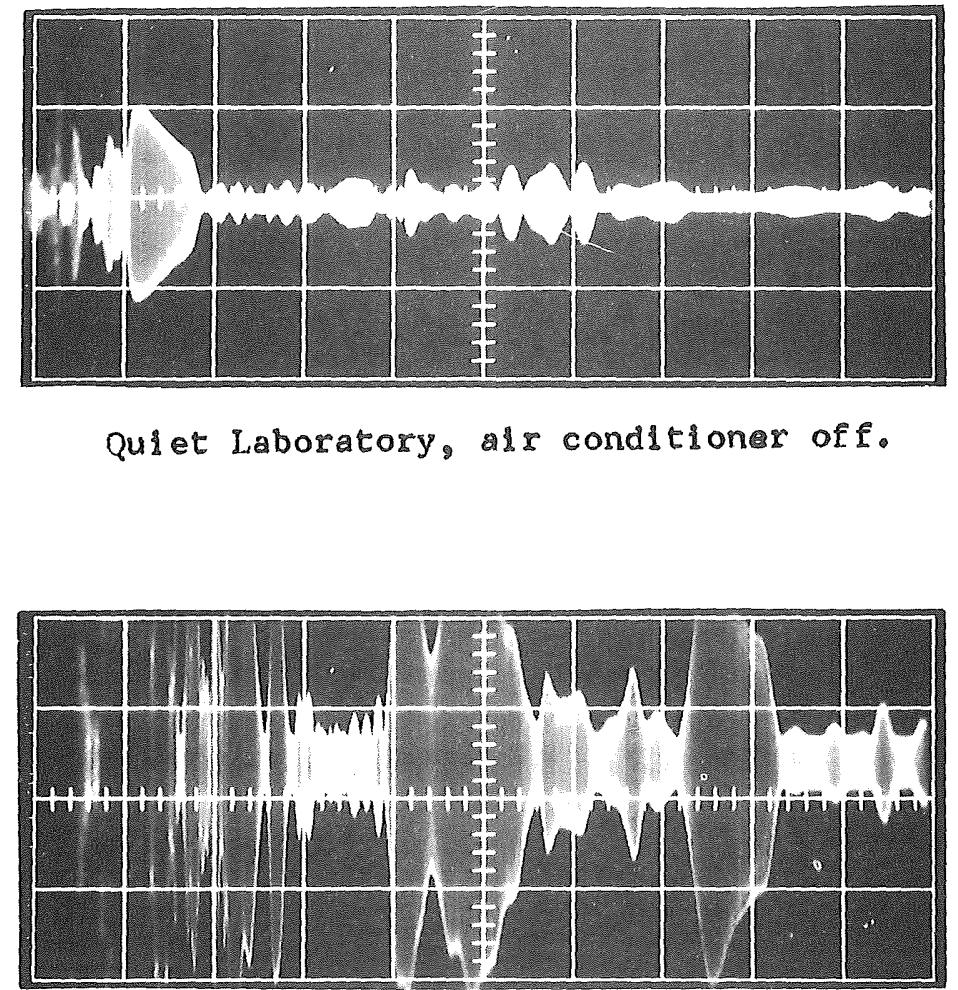

Laboratory, alr conditioner on.

Flgure 41. Typical turbulencemodulated carrier.signals. 





TABLE IV

SAMPLE RESULTS: $\mathrm{C}_{\mathrm{n}}{ }^{2}$ DETERMINATION BY THREE METHODS

\begin{tabular}{|l|c|c|c|}
\hline No. & A & B & C \\
\hline & From & From & From \\
& $\left\langle(\Delta \mathrm{T})^{2}\right\rangle$ & $\Delta \mathrm{T}$ & $500^{\prime}$ \\
& & Probability & Scintillations \\
\hline 1 & 7.2 & - & - \\
2 & 36 & 5.3 & 10 \\
3 & 22 & 1.8 & 3.0 \\
4 & 31 & 1.2 & 7.1 \\
5 & 4.6 & 1.4 & 3.7 \\
\hline 6 & 2.3 & 0.22 & 0.61 \\
\hline
\end{tabular}


the slope of the distribution (Figure 42). The time recordings of $\mathrm{C}_{\mathrm{n}}{ }^{2}$ values (Figure 43) obtained simultaneously by optical means (portable laser at 500 feet, where the non-saturation theory may be expected to hold), and thermally, show fair agreement of the methods. Some results from the two methods show a considerable spread between each other. The matter is under further study. The line which represents the far-infrared is also seen to be in fair agreement (Chapter II- $D$, 2-26), whereas the visible wavelength is already saturated, and shows an increasingly negative correlation, due to the negative slope of the saturation curve (Chapter II, Figure 2). 


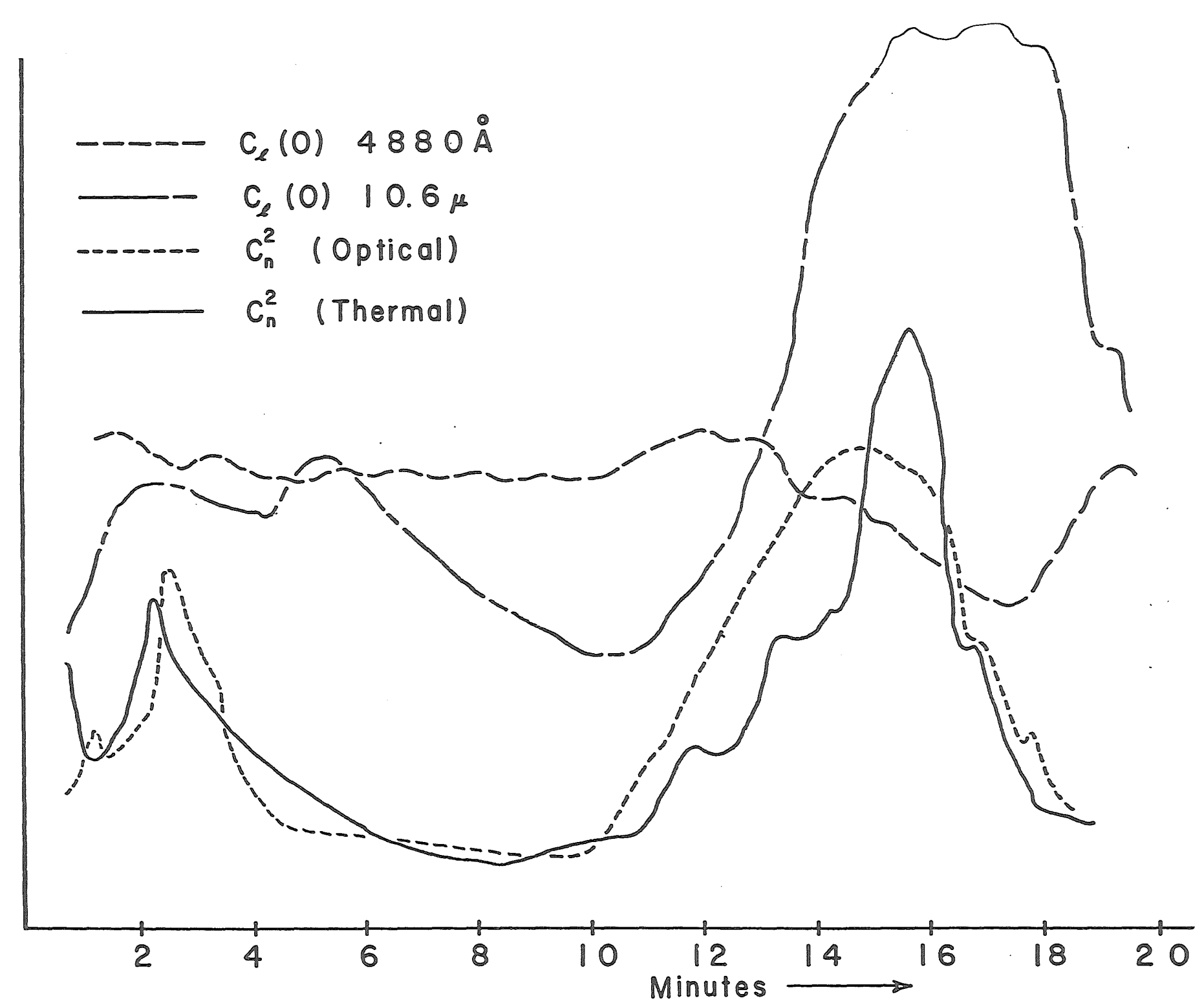

Figure 43. Recording of $\mathrm{C}_{\mathrm{n}}{ }^{2}$ and scintillation variances vs time 
BIBLIOGRAPHY 


\section{BIBLIOGRAPHY}

1. Lowry, W. P. and Boube1, R. W., "Meteorological Concepts in Air Sanitation", Oregon State University, Corvallis, Oregon.

2. Kuo, B. C., Analysis and Synthesis of Sampled-Data Control Systems, Prentice-Ha11, Inc., Englewood Cliffs, New Jersey, 1963.

3. Gödecke, K., "Messungen der atmosphärischen Turbulenz", Ann.d. Hydrographie, $\underline{10}, 400,1936$.

4. Rytov, S. M., "Diffraction of light by ultrasonic waves", Izv. Akad。Nauk SSSR, Ser.Fiz., No.2, 223, 1937.

5. Loitsyanski, L. G., "Some basic laws of isotropic turbulent flow", Trudy Aero. Gidro. Inst., No. 440, Moscow, 1939. Translated into English as Tech. Memo. No. 1079, Nat. Adv. Comm. Aero, Washington, D.C.

6. Obukhov, A. Mo, "On the scattering of sound in a turbulent flow", Dok1ady Akad. Nauk SSSR, 30, 611, 1941.

7. Kolmogorov, A. N., "Dissiptation of energy in locally isotropic turbulence", Doklady Akad. Nauk SSSR, 32, 16, 1941. German trans lation in "Sammelband zur Statistischen Theorie der Turbulenz", Akademie-Ver1ag, Ber1in, 1958, p. 77.

8. Batchelor, G. K., "The Theory of Homogeneous Turbulence", Cambridge Univ. Press, 1953.

9. Tatarski, V. I., Wave Propagation in a Turbulent Medium, Trans., Dover Publications, Inc., New York, 1967.

10. Zwang, L. R., "Measurements of Temperature Pulse Frequency Spectra in the Surface Layer of the Atmosphere", Izv. Geophys. Ser., 1960, pp. 1252-1262.

11. Hufnage1, R。 E. and Stanley, N. R., "Modulation Transfer Function Associated with Image Transmission Through Turbulent Media", J. Opt. Soc. Am., vo1.54, pp. 52-61, January 1964 .

12. Davis, J. I., "Consideration of Atmospheric Turbulence in Laser System Design", Applied Optics, No.1, pp。139-147, January 1966.

13. Fried, D. L., "Optica1 Heterodyne Detection of an Atmospherically Distorted Signal Wave Front", Proc. IEEE, pp. 57-67, January 1967. 
14. DeWo1f, D. A., "Saturation of Irradiance Fluctuations Due to Turbulent Atmosphere", J. Opt.Soc.Am。, vol. 58, pp.461-466; Apri1 1968.

15. Ochs, G. R., "A Resistance Thermometer for Measurement of Rapid Air Temperature Fluctuations", ESSA Technical Report IER 47-ITSA 46, October 1967.

16. Ochs, G. R., "Laser-Beam Scintillation over Horizontal Paths from 5.5 to 145 Kilometers", J.Opt. Soc. Am., vol. 59, pp. 231-234, February 1969 .

17. Wright, N. Jo and Schutz, R. J., "Measurement of the Refractive Index Structure Coefficient C ", Memorandum Report No. 1885, Ballistic Research Laboratories, Aberdeen Proving Ground, Maryland, December 1967.

18. Kerr, J.R., "Proposal for a One-Year Study of the Propagation of 10.6-Micron Radiation Through Atmospheric Turbulence", Oregon Graduate Center, Portland, Oregon, December 1967.

19. Strohbehn, J.W., "Line-of-Sight Wave Propagation through the Turbulent Atmosphere", Proc. IEEE, vol. 56, pp.1301-1318, August 1968 .

20. Kerr, J.R., "Proposa1 for a One-Year Study of the Propagation of Multiwavelength Radiation Through Atmospheric Turbulence", Oregon Graduate Center, Portland, Oregon, March 1968.

21. Livingston, P.M., Deitz, P.H. and Alcaraz, E。C., "Coherent Light Propagation Through a Turbulent Atmosphere: Measurements of the Optical Filter Function", submitted for publication to the J. Opt. Soc. Am., August 1969 .

22. Debye, P., "Polar Molecules", Dover Books on Physics, Engineering, Dover Publications, Inc., pp. 63-69, pp. 27-ff., 1929.

23. Brown, C. M., "The Electronic Instrumentation for Atmospheric Laser Propagation Studies", Master's Thesis, Portland State College, 1968.

24. Handbook of Operationa1 Amplifier Applications, Burr-Brown Research Corporation, Tucson, Arizona, 1963.

25. Applications Manua1 for Computing Amplifiers for Modelling, Measuring, Manipulating and Much Else", Philbrick Researches, Inc., Dedham, Massachusetts, 1966.

26. Analog Devices, "Application Notes", Cambridge, Massachusetts, 1969. 
27. "Flow Corporation Hot-Wire Probes Operating Instructions", Bulletin 516-HWP-P, Flow Corporation, Waterton, Massachusetts, 1968 .

28. Kerr, J. R., Private Communication, Oregon Graduate Center, Portland, Oregon.

29. Gibbons, J. F. and Horn, H. S., "A Circuit with Logarithmic Transfer Response Over 9 Decades", IEEE Transactions on Circuit Theory, September 1964 .

30. Sto11, J. L., Private Communication, Tektronix, Inc., Beaverton, Oregon.

31. Verhoef, W., Private Communication, Tektronix, Inc., Beaverton, Oregon.

32. Kerr, J. R., "Multiwavelength Laser Propagation Study -- II", Quarterly Progress Report No. 3, Oregon Graduate Center, Portland, Oregon, April 1970.

33. Lawrence, R. S., Private Communication, ESSA, Boulder, Colorado.

34. Deitz, P. H., Private Communication, University of Washington, Seattle, Washington. 
APPENDICES 


\section{APPENDIX A \\ SIMPLE LOGARITHMIC GONVERTER WITH GAIN \\ USING ONE OPERATIONAL AMPLIFIER}

For applications requiring only three decades of dynamic range, a less expensive logarithmic converter was realized and tested. The cir= cuit is shown in Figure 44. A transistor is again used as the feedback element and a diode protects it from reverse voltage. However, the operational amplifier is first followed by a current amplifier in order to allow the use of a low-resistance divider before the feedback transistor. This measure reduces errors between the divider input and its tap. The errors can be expressed as the ratio of the resistor $\mathrm{R}$ to the transdiode emitter-base resistance $r_{e}$. Neglecting second-order effects, this gives:

$$
\text { Error } \%=100 \frac{\mathrm{R} I_{\text {in }}}{\mathrm{kT} / \mathrm{q}}=3900 \mathrm{R} \mathrm{I}_{\text {in }} \quad\left(I_{\text {in }}\right. \text { amps) }
$$

Allowing an error of five percent results in an upper current limit of

$$
I_{\text {in }}=0.1310^{-2} / \mathrm{R}
$$

and with the shown value of resistance (51 ohms) $I_{\text {in }}=25.5$ microamperes 。

This result confirms that a range of three to four decades can be achieved. If care is taken with offset voltage and bias current compensations, and if temperature variations are kept under control, good accuracy is obtained. 


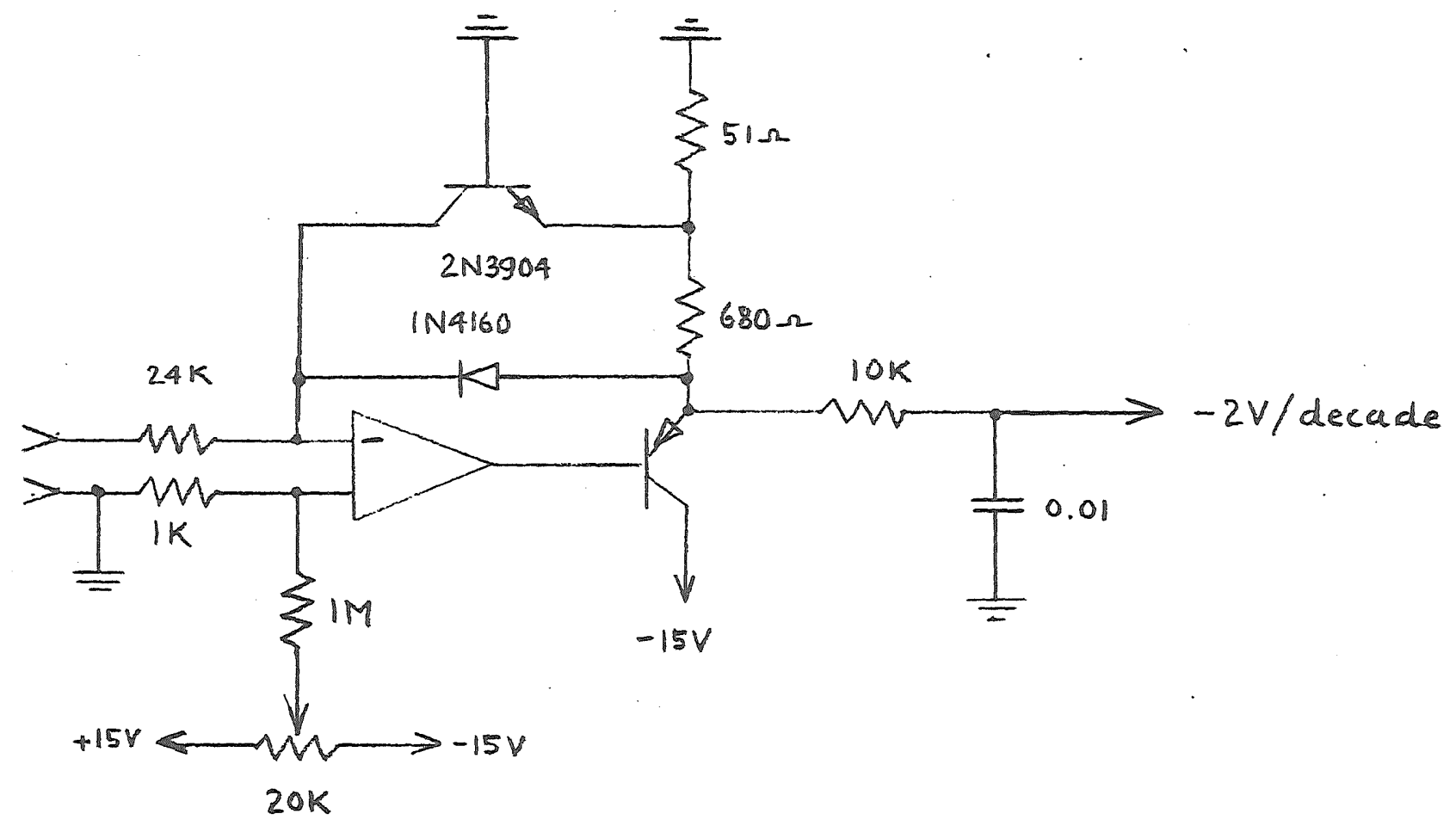

Figure 44. Single-operational-amplifier log-converter 


\section{APPENDIX B}

\section{STABILITY OF THE LOGARITHMIC CONVERTER}

Figure 45 shows an operational amplifier (gain $=\frac{A_{0}}{1+s \tau_{a}}$ ) and a transistor connected to its input. The transistor equivalent circuit is simplified by assuming the base resistance is zero. The emitter resistor $r_{e}$ is later joined to the amplifier output; but for the stability analysis a voltage $V_{e}$ is applied and Kirchoff's law is written for the input node

$$
\varepsilon\left(y_{\text {in }}+y_{c}+c_{c} s\right)=v_{e} \alpha_{o} / r_{e}
$$

hence

$$
\frac{v_{e}}{\varepsilon}=\frac{1}{\beta(s)}=\frac{r_{e}\left(y_{i n}+y_{c}\right)\left(1+\frac{c_{c} s}{y_{i n}+y_{c}}\right)}{\alpha_{0}}
$$

The magnitude of the ratio $\frac{V_{e}}{\varepsilon}$ is plotted versus frequency in the Bode plot (Figure 45), along with the amplifier gain.

If the closed loop is to be stable, a simple rule is that the closing rate between the two lines $A(s)$ and $\beta^{-1}(s)$ must not exceed $6 \mathrm{~dB} /$ octave. This assures a phase shift no greater than $-90^{\circ}$. Since this condition is not satisfied, the feedback network is parallelled with a capacitor $\mathrm{C}_{\mathrm{f}}$, creating a zero:

$$
\varepsilon\left(y_{i n}+y_{c}+C_{c s}+C_{f s}\right)=V_{e}\left(\alpha_{o} y_{e}+C_{f s}\right)
$$



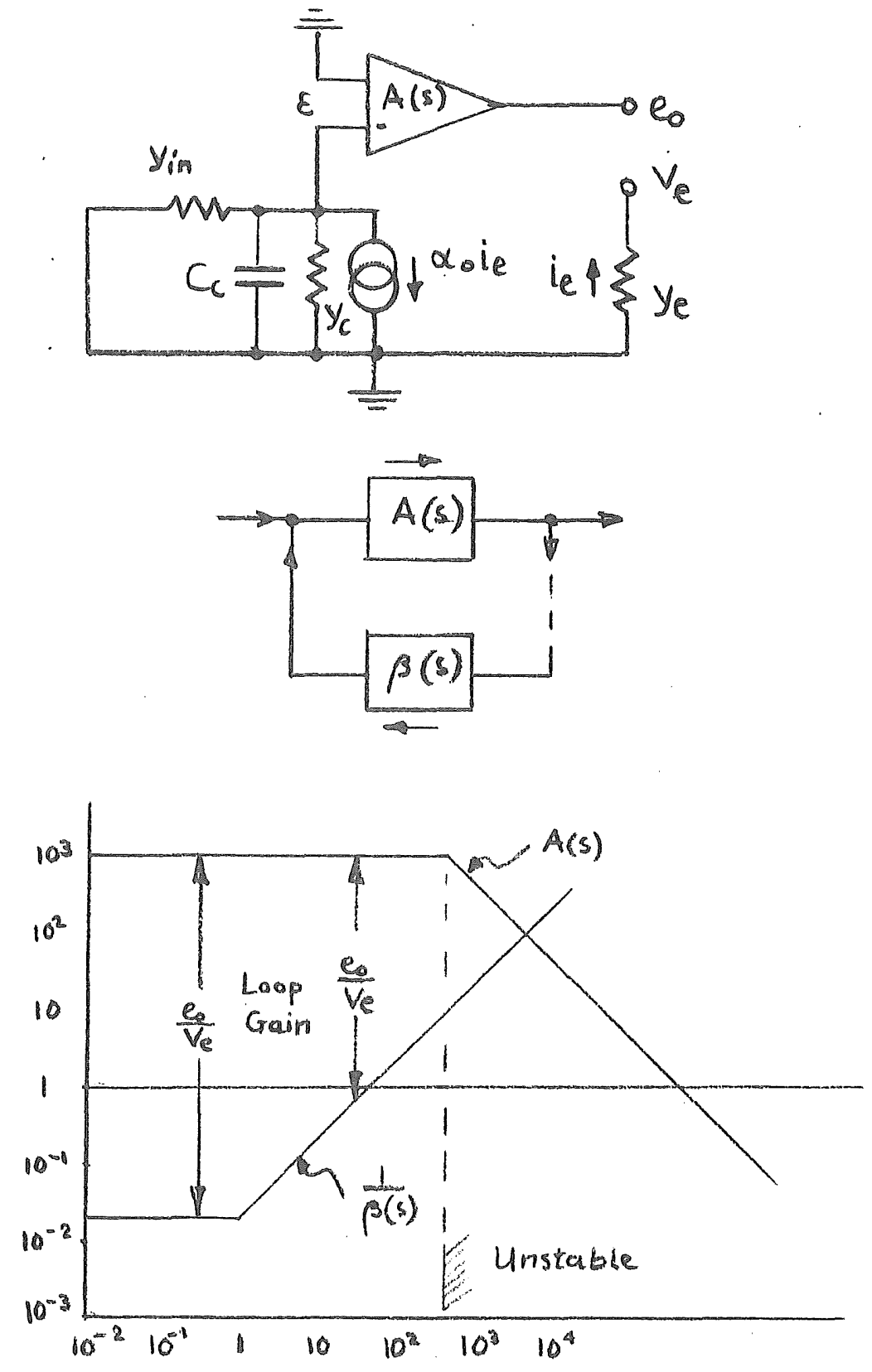

Figure 45. Logarithmic converter open-1oop gain 
Whence (Figure 46)

$$
\frac{v_{e}}{\varepsilon}=\frac{1}{\beta(s)}=\frac{\left(y_{i n}+y_{e}\right)\left[1+\frac{C_{c} s+C_{f} s}{y_{i n}+y_{e}}\right]}{\alpha_{o} y_{e}}
$$

where

$$
\begin{aligned}
& \mathrm{V}_{\mathrm{e}}=\text { auxiliary open-loop input voltage } \\
& \beta(s)=\text { feedback branch transfer function } \\
& \mathrm{y}_{\text {in }}=\text { input conductance } \\
& \mathrm{y}_{\mathrm{e}}=\text { emitter-branch conductance } \\
& \text { (including emitter-base conductance) } \\
& \mathrm{C}_{\mathrm{c}}=\text { collector-base (ground) capacity } \\
& \mathrm{C}_{\mathrm{f}}=\text { stabilizing feedback capacity }
\end{aligned}
$$

The capacitor $\mathrm{C}_{f}$ is so selected that the corresponding zero occurs before or at the amplifier pole. Then the overall Bode characteristic can be seen to correspond to a single-pole device. As an alternative, the amplifier gain may be reduced by adding $R_{f}$ and $R_{0}$ to the operational amplifier (Figure 46). Thus, the corner frequency is increased to accommodate a lower value for $C_{f}$ in the interest of frequency response.

The above method of stabilization is discussed in more detail in the literature (29). 

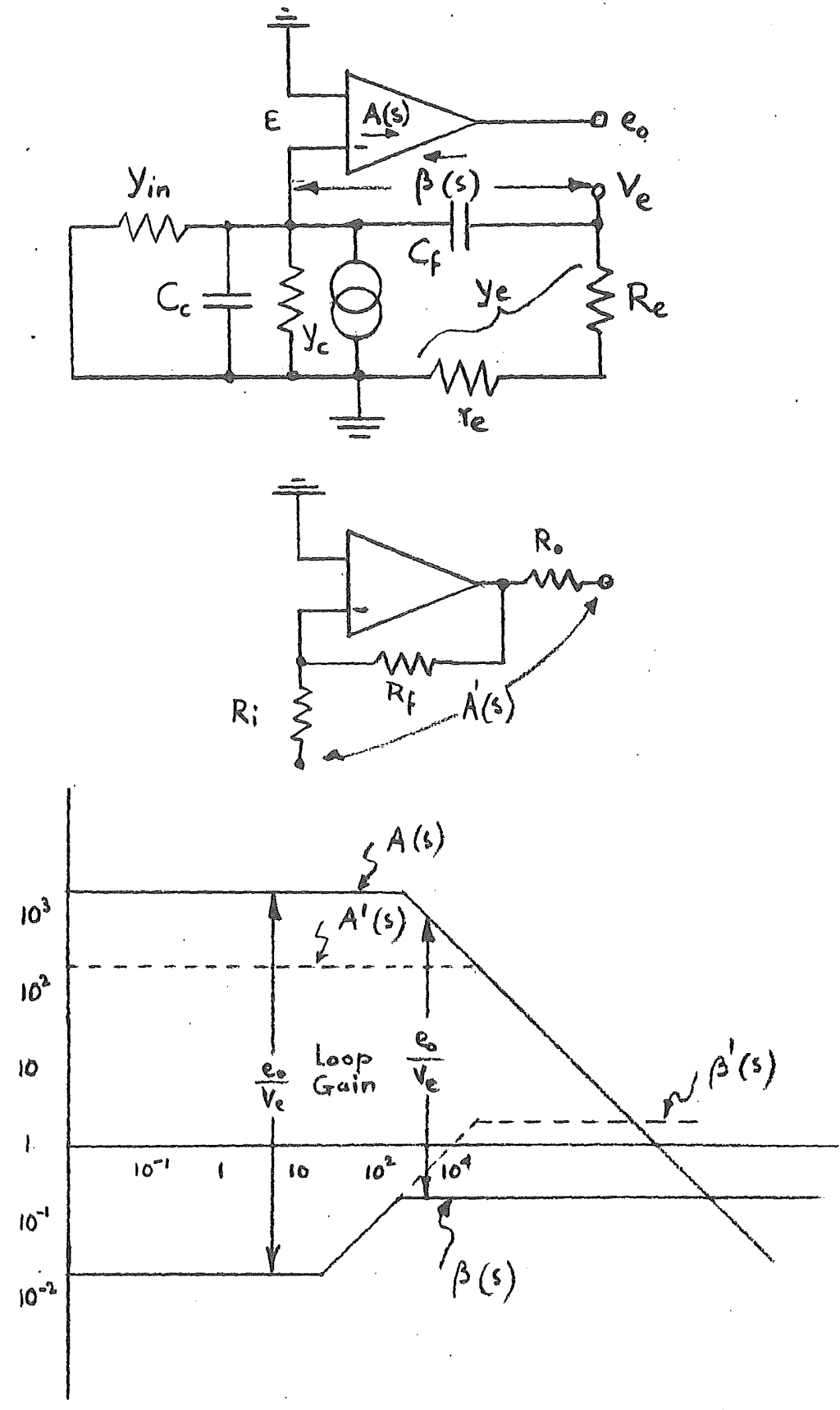

Figure 46. Logarithmic converter, corrected open loop gain 
APPENDIX C

\section{CALCULATION OF THE MAXIMUM ADMISSIBLE DIODE SATURATION CURRENT \\ IN THE ABSENCE OF NOISE}

In order to get an idea of the relative magnitude of effects which reduce the signal-to-noise-ratio, it is desirable to evaluate the performance of the diodes in the precision envelope demodulator. Perfect rectification is made impossible by the following causes: leakage current, reactive bypassing, and excessive forward diode resistance. Since the diodes are located in the feedback path, the gain is affected by their dynamic resistance.

When the forward current in a diode decreases, its resistance becomes larger and it may exceed the value where the well-known approximation for voltage gain holds $\left(G=Z_{f} / R\right.$ when $K \gg Z_{f} / R$ ). When $R_{f}$ (the diode resistance) increases, the ratio $Z_{f} / R$ approaches $K_{\text {, }}$ and one must use the accurate formula for voltage gain:

$$
G=\frac{Z_{f}}{\frac{Z_{K}}{K}\left(1+\frac{R}{R_{i n}}\right)+\frac{R}{K}+R}
$$

where

$$
\begin{aligned}
G= & \text { voltage gain } \\
Z_{f}= & \left(R_{E}+1 / j C_{f} w\right) \\
C_{f}= & \text { total feedback capacity (across the two } \\
& \text { diodes, stray capacity) } \\
R= & \text { input resistance }
\end{aligned}
$$




$$
\begin{aligned}
\mathrm{R}_{\text {in }}= & \text { resistance between operational amplifier } \\
& \text { input terminals } \\
\mathrm{K}= & \text { operational amplifier voltage gain (open loop) }
\end{aligned}
$$

The bypass current through the capacity $C_{f}$ is $A C$. It has a mean value of zero, and therefore is lost as far as rectifier efficiency is concerned. We shall impose on $C_{f}$ the condition that it must equal the absolute value of the diode forward resistance $R_{f}$ when the maximum permissible gain reduction resulting. from high $R_{f}$ or $Z_{f}$ is reached.

Diode reverse leakage current subtracts from the average rectified current and, thus, also reduces envelope detection efficiency at low carrier amplitude.

These three impediments of demodulation efficiency are assigned equal shares of the 30\% maximum tolerable loss, which defines the limit of sensitivity of the (noiseless) instrument.

To guarantee no more than $10 \%$ loss by leakage, the minimum forward current is defined as 10 times the diode saturation current:

$$
I_{\mathrm{d}}=\mathrm{pI}
$$

where

$$
\mathrm{p}=10
$$

The static diode resistance is found by solving the diode equation (3-2) for $V$ and dividing it by the current $I_{d}$ :

$$
R_{s t}=\frac{V}{I_{d}}=\frac{k T}{q} \frac{\ln (p+I)}{I_{s} p}
$$

The limiting value for the static diode resistance, determined by the above argument, is given by permitting the 10\% gain reduction: 


$$
\mathrm{Z}_{\mathrm{f}}=\frac{0.1(1+\mathrm{K}) \mathrm{R}}{0.9}
$$

so that

$$
R_{f}=\frac{0.1(1+K) R}{0.9} 2
$$

The diode saturation current is thus given by equating $(C-3)$ and $(C-5)$ :

$$
I_{S}=\frac{0.9 k T \ln (p+1)}{0.141(1+K) q p R}
$$

which yields for the numerical values given $\left(\mathrm{K}-10^{5}, \mathrm{R}=620 \mathrm{ohms}\right.$, $\mathrm{p}=10)$

$$
I_{S}=0.655 \text { nanóamperes }
$$

Between the lowest operating current $(6.65 \mathrm{nA})$ and the highest peak operating current, given by the maximum undistorted voltage swing, of which the amplifier is capable:

$$
I_{m}=16.1 \mathrm{~mA}
$$

extends a dynamic range of $24.3 \times 10^{6}$ (noiseless condition). 


\section{APPENDIX D}

\section{AMPLITUDE CALIBRATOR FOR THE SCINTILLATION COMPUTER}

In order to test the non-linear conversion modules (demodulator, $\log$ converter, and multiplier) an amplitude calibrator (Figure 47) was constructed by the author. A conventional multivibrator drives an amplifier of moderate voltage gain into positive and negative saturation. Internal short circuit protection of the operational amplifier permits the clamping of its output to ground, during the negative output cycle, by means of a zener diode $D_{1}$.

The constant-output-impedance, precision decade divider is fed through diode $D_{2}$ which disconnects the driving source during the -0.6 volt interval (clamped to ground by forward biased zener diode). The output impedance of the 10 volt tap is $9.11 \mathrm{k} \Omega$, all others are $830 \Omega$. During the positive period, the zener diode $D_{1}$ limits the amplifier output to $10.6 \mathrm{~V}$, at a very low output impedance $\left(\mathrm{Z}_{0}^{1}\right)$, given by:

$$
Z_{0}^{\prime}=\frac{Z_{0}}{G_{0}}
$$

where $Z_{o}$ is the open loop output impedance and $G_{0}$ is the loop gain. The resistor of $886 \Omega$ between the zener diode $D_{1}$ and diode $D_{2}$ gives the first tap an output impedance equal to that of all other taps, namely, $830 \Omega$, during every positive period, so that no error occurs on any tap while output current is supplied. During the off periods, the different output impedance on the highest tap causes no error in output voltage. 


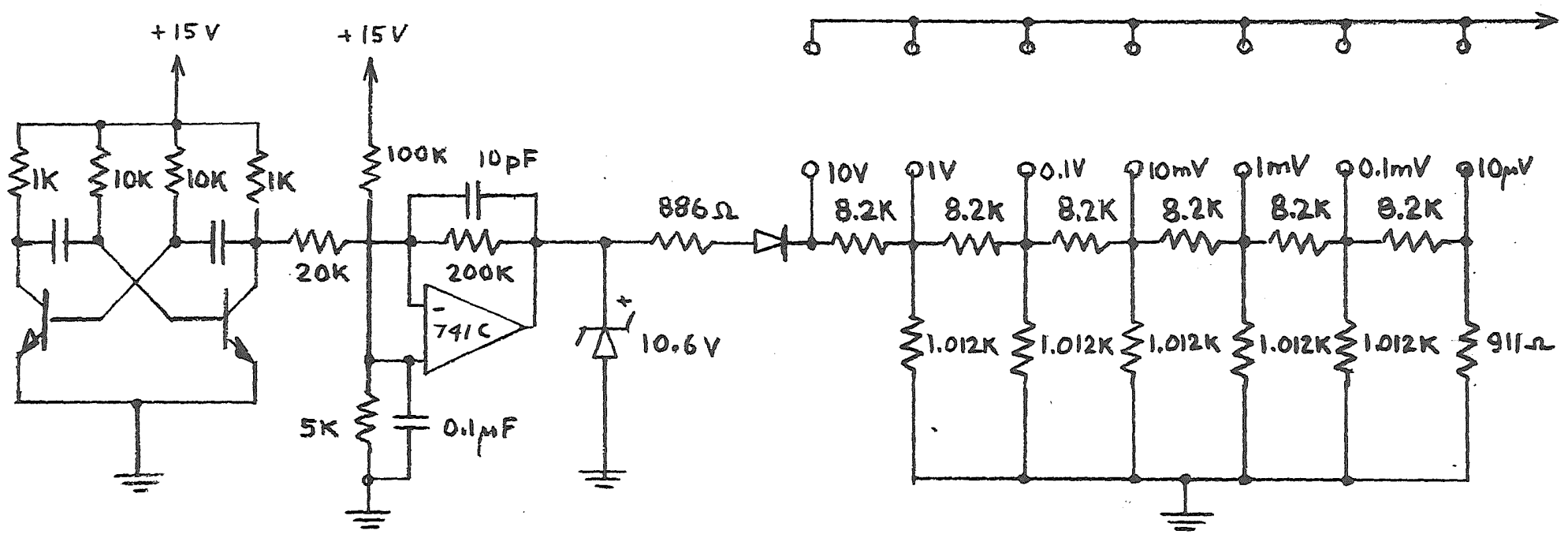

Figure 47. Amplitude calibrator for logarithmic converter 


\section{APPENDIX E \\ LOOP GAIN COMPENSATION AND STABILITY \\ IN THE $\mathrm{C}_{\mathrm{n}}$ UNIT}

As, the gain of the differential thermometer is increased, the feedback control loop gain must be kept constant in order to preserve the damping ratio (Figure 48). Essentially what is needed is a variable voltage divider between the output of the low-pass filter after the demodulator, and a pure DC source of the same potential as the averaged filter output; or alternatively, the input resistor of the control amplifier may be changed to make the product of signal gain and control amplifier gain constant. In the first case, the source potential must be very accurately trimmed, and the control amplifier offset errors are critical. In the second case, the control amplifier leakage becomes influential in setting the signal level. In order to avoid these shortcomings, a hybrid solution combining the two above methods was adopted.

Each of the methods now needs to cover a variation of on $1 \mathrm{y} 31.6$ to 1. This leaves the lowest tap of the potentiometric divider near enough to the filter output that its changes have adequate control over the offset or adjustment errors, because compared to the aforementioned conditions, the lowest tap has a 31.6 times higher voltage variation. For highest signal gain (and highest control-amplifier gain reduction), the series or input resistor to the control amplifier must now be on $1 \mathrm{y} 1 / 31.6$ of the value required without the voltage 
To bridge control lamps

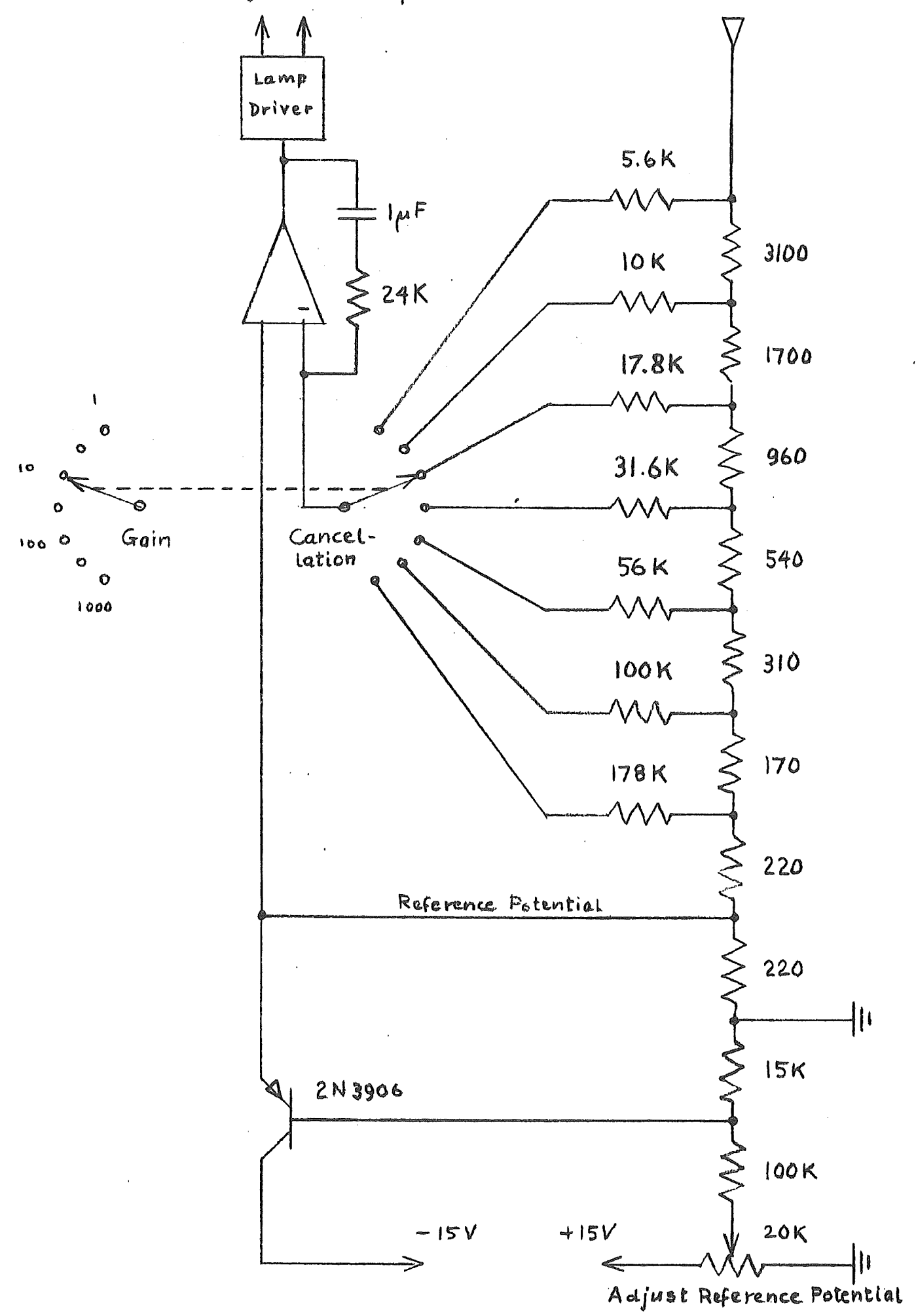

Figure 48. Galn Cancellation in control loop of $\mathrm{C}_{n}{ }^{2}$ unit. 
divider, and the leakage currents cannot build up enough potential to interfere with the control 1oop.

The low (or DC) end of the divider is returned to the noninverting terminal of the control amplifier, which is held at the adjustable reference potential. Of course, at any gain position, the filter output average is held at that leve1. The higher the gain is set, the nearer the bridge will be to true balance. At low gains, the bridge is kept further away from this nu11, thus accommodating the larger dynamic changes in sensor imbalance. 


\section{APPENDIX F \\ EFFECT OF THE WIRE PROBE DIAMETER AND LENGTH \\ ON THE SENSITIVITY OF THE $C_{n}$ UNIT}

The heat capacity of a body is proportional to the product of its mass and the specific heat of the material from which it is made.

$$
\frac{\Delta Q}{\Delta T}=V c s
$$

where

$$
\begin{aligned}
\Delta Q & =\text { incremental heat energy in calories } \\
\Delta \mathrm{T} & =\text { incremental temperature } \\
\mathrm{C} & =\text { specific heat of the material } \\
\mathrm{S} & =\text { specific density of the material } \\
\mathrm{V} & =\text { volume }
\end{aligned}
$$

Heat flows in and out of a body through its surface in the form of radiation or convection. There is no heat exschange when the body and its environment are at the same temperature. When a sudden temperature change occurs, the heat flow depends on the surface offered, on the temperature difference, and on thermal conduction of body and environment. The time after which equilibrium is approached to a given degree depends on the mass of the body. For a wire of length 1 and diameter $2 r$, for instance, the initial rate of heat flow is:

$$
\frac{\Delta Q}{\Delta t}=\mathrm{K}(\mathrm{T}) \mathrm{r} 1
$$

where $\mathrm{K}=2 \mathrm{~K} \pi$. 
The change of temperature with time is given by:

$$
\frac{\Delta T}{\Delta t}=\frac{\Delta Q}{V \operatorname{cs} \Delta t}=\frac{K r 1}{\operatorname{cs} 1 r^{2} \pi}=\frac{2 K_{0}}{\operatorname{csr}}
$$

For wires of equal length, but radii $r_{1}$ and $r_{2}$, and subject to the same temperature change (step) of the environment, the initial temperature increments are given by the ratio:

$$
\frac{\Delta \mathrm{T}_{1}}{\Delta \mathrm{T}_{2}}=\frac{\mathrm{r}_{2}}{\mathrm{r}_{1}}
$$

The above equation says that a smaller radius will result in a higher temperature sensitivity and that the relation is reciprocal.

The current requirements for wires of different diameters for a given smal1 temperature rise above the environmental temperature is now considered. Let $\mathrm{T}$ be the small temperature increase reached by the thick wire $\left(r_{2}\right)$ when the energy input equals the energy loss through radiation and convection, both of which are proportional to the wire surface. Let the current $I_{2}$ in the thick wire of radius $r_{2}$ be known. Then the energy input is given in terms of $r_{2}$, by:

$$
W_{i}=I_{2}^{2} R=I_{2}^{2} \frac{p 1}{r_{2}{ }^{2} \pi}
$$

The radiated energy is given by:

$$
W_{r}=K(T) 2 \pi r_{2} 1
$$

Equality of these equations requires:

$$
\mathrm{I}_{2}{ }^{2}=2 \mathrm{~K}(\mathrm{~T}) \mathrm{r}_{2}{ }^{3} \mathrm{r}^{2} \rho^{-1}
$$


where

$$
P=\text { specific resistance of the wire. }
$$

The last equation holds also for the wire of radius $r_{1}$, with the current $I_{1}$.

$$
I_{1}^{2}=2 \mathrm{~K}(T) r_{1}{ }^{3} \pi^{2} \rho^{-1}
$$

Hence, the currents are in the ratio:

$$
\frac{I_{1}}{I_{2}}=\left[\frac{r_{1}}{r_{2}}\right]^{3 / 2}
$$




\section{APPENDIX G}

\section{REPAIR OF DAMAGED THIN PROBE-WIRES}

In this Appendix, we describe two techniques for replacing the fine wires on the probes of the $C_{n}$ unit.

\section{A. TECHNIQUE 1}

\section{Preparation of Probe Tips}

Remove the old wire and clean the needle supports with a fine file or emery cloth. Produce a small flat at the tips of the needles. Using Allstate $430 \mathrm{flux}$, and Allstate 430 solder, tin the tips. This is done by wetting the tips slightly with the flux. Melt a very small amount of solder onto the tip of a $1 \mathrm{~mm}$ thick soldering bit and touch it first to the surface of the flux to make the solder shiny by removing the oxide coat, then to the two needle support tips.

Proceed in the same manner as above when tinning the approximately $5 \mathrm{~mm}$ length of Wollaston process wire (which is drawn inside a silver jacket to its desired thickness). Do not, however, immerse the wire in the flux at a11. The heated chemical would etch off the silver during the short duration of the tinning, by a touch of the soldering bit. Just draw the wire a couple of times across the cleaned, slightly tinned bit.

\section{Soldering}

Barely moisten one of the needle support tips with a minute trace 
of flux. While observing under a 10 to 20 times magnifier, hold the end of the Wollaston process wire on the support tip and touch the latter a few millimeters beyond with the soldering iron. This causes the tin to melt around the end of the wire, thus producing a neat junction. Next, gently bring the wire in contact with the other needle support tip so that it touches without being pulled or forced. The wire should not be subjected to stress or strain. Touch the supporting needle a few millimeters from its tip with the soldering iron, making sure that a good junction results. Then cut the loose ends of the wire, without touching or bending the needles. Dress the remaining ends, if too long.

\section{Protective Coating of Supports}

The silver must now be etched away without weakening the soldered junctions, as these are more easily etched than the free portion of the wire. To avoid this, dissolve a small amount of Apiezon "black wax" in trichlorethylene and paint the portions which must be protected, using

a fine sable brush. In order to avoid damage to the wire, do not touch it. As an alternative, the needles may be immersed, one at a time, into the wax solution to apply a coating around the wire-to-tip junctions and along the needle supports.

\section{Etching}

To etch the silver jacket off the wire after the probe has been prepared in this manner, fasten it in a vise, with the needles pointing slightly downward. Using laboratory strength nitric acid solution (one part concentrated acid and five parts water), the etching is done 
as follows $(33,34)$ : Top a pen-size, very thin pipette with a rubber finger. Pick up a bit of acid so that when the rubber is free, the acid will be at, or near, the bottom end of the pipette. While observing under a magnifier, advance the pipette tip to the (horizonta1) wire to be etched, and squeeze the acid to form a sma11, 3/4drop, still well attached to the tip of the pipette. With steady hand, using a comfortable support and leverage, move the drop into the wire, and then along the wire. Never come so close to the needle supports that the drop wraps itself around them. Within a short time the silver is removed and the bare platinum or platinum rhodium is seen. Continue to etch for 30 to 60 seconds, always staying away from the junctions.

\section{Cleaning}

Using a test tube filled with water, carefully dip the etched wire into the water and gently move it back and forth to wash off the etching chemicals. Repeat this procedure several times. In order to wash off the black wax, carefully (and liberally) squirt trichlorethylene (from a squeeze bottle) over the covered parts until all wax is removed. Then wash in alcohol and let dry.

\section{B. TECHNIQUE 2}

\section{Cementing}

The second method has not been tried here, but it was described to the writer (33).

In this method, the wire is first etched over a length of 6 to $8 \mathrm{~mm}$, washed in water and alcohol, and then dried. The probe tips are 
likewise prepared for the repair. Then they are dipped in a slightly spread out drop of silver paint (like General Cement No. 212), and, thus prepared, are used to pick up the wire. The wire sticks to the paint, which joins it conductively to the needle support tips. Allow to dry. After drying, snip off loose ends .

Verification of Results

Before the probe is installed for use, it is suggested that the following tests be made:

Mechanica1. Measure diameter of wire by means of a diffraction pattern to make certain that all silver has been removed.

Electrica1. Do not check continuity with an ohmmeter without making certain that the test current is limited to a safe value by series resistance. The exact resistance of the wire can be measured by forcing a constant current of $500 \mu \mathrm{A}$ through it and measuring the potential difference with a VTVM (sensitive voltmeter). The impedance of this voltmeter should be at least $10 \mathrm{k} \Omega$ for $1 \%$ accuracy. Now the probe is ready for use. 


\section{APPENDIX H}

LARGE TIME CONSTANT IN FEEDBACK INTEGRATORS

In order to obtain large integrating time constants in averagers, the product of feedback resistor and capacitor must be large. This leads to large values of both; therefore, it is necessary to discuss the limitations which govern the choice of these components.

The DC gain of a feedback amplifier is given by:

$$
G=\frac{\frac{R_{f}}{R}}{\frac{R_{f}}{K R}+\frac{R_{f}}{K R_{i n}}+\frac{1+K}{K}}
$$

If $K \gg \frac{R_{f}}{R}$, then the denominator reduces practically to unity. However, when high voltage-gains are desired, $R_{f} / R$ approaches $K$. When both are of equal magnitude, the gain is only half of the ratio $R_{f} / R$ or of $\mathrm{K}$. This means that the gain is as much determined by the factor $\mathrm{K}$ as by the ratio of feedback to input resistance. Since $\mathrm{K}$ is usual1y specified within a factor of 5 or more $\left(\mathrm{K}_{\min }=10^{5}, \mathrm{~K}_{\max }=5 \times 10^{5}\right)$, the actual gain in the feedback amplifier could vary by a factor of 1.8. Therefore, one limits the ratio $R_{f} / R$ to a small fraction of $K$ $(2-3 \%)$, so as to minimize dependence of gain on $\mathrm{K}$.

The size of $R_{f}$ is proportional to $R$, which depends on the power capabilities of the input generator. If the input signal is the output of an operational amplifier, $R$ may be between 2 and $5 \mathrm{~K}$. 
Without leakage, $R_{f}$ could be 8-20 M . Leakage considerations reduce this to a value given by the maximum allowed error voltage $\left(V_{e}\right)$, in terms of leakage current $\left(I_{1}\right)$.

$$
R=V_{e} / I_{1}
$$

Therefore, one is tempted to use larger capacitors. Unfortunate$1 y$, these have also leakage currents, usually not equal in both current directions. For simplicity, the leakage is assumed to be the same in both directions. Its magnitude increases with capacity. This can be expressed in terms of parallel resistance, say, $R$ ohms per microfarad. Generally, regardless of its value in microfarads, such a capacitor has then an inherent time constant $\left(\tau_{0}, s e c\right)$, equal to the numerical value of $R$, when $R$ is in $M \Omega$. Since the leakage resistance depends on age, temperature, and other varying factors, one should not attempt to realize time constants approaching this value. The highest time constant that one can obtain depends on the accuracy within which it must be specified. For a given accuracy ( $\%$ ), a fixed resistor $r$ must be associated with the capacitor (and in parallel with the leakage resistance), the value of which must not exceed $p \%$ of $R_{1}$, the leakage resistance.

$$
\mathrm{R}_{1}=\frac{\mathrm{R}}{\mathrm{A}}
$$

where $\mathrm{A}$ is the number of $\mu \mathrm{F}$ contained in the capacitor. It is clear that an increase in the capacity by a factor A would require a decrease of $r$ by the same factor; therefore, it is desirable 
to choose the smallest capacity compatible with $\Gamma_{\text {, when }} r$ is taken as large as possible, subject to the considerations discussed in the previous paragraph on feedback resistance. The only freedom one has, is to choose a high-quality, low-leakage capacitor and a high-gain amplifier with good offset current compensation. These components allow the use of high feedback resistance and larger capacitance. 